\title{
Ideais Primários em Anéis de Witt
}

\author{
Daniela Cristina Rebolho
}

Orientadora: Profa. Dra. Ires Dias

Dissertação apresentada ao Instituto de Ciências Matemáticas e de Computação - ICMC-USP, como parte dos requisitos para obtenção do título de Mestre em Ciências - Área: Matemática.

USP - São Carlos

Março de 2000 
"À minha família." 
Inicialmente agradeço À DEUS, pelo dom da vida e por ter me dado forças suficientes a fim de superar os obstáculos e conquistar meu objetivo. Agradeço a Prof $^{2}$. Dr ${ }^{2}$. Ires Dias, pela sua dedicada e precisa orientação, e por sua amizade; a FAPESP pelo custeio parcial de meus estudos de Pós-Graduação; aos meus pais Dirceu e Maria, meu irmão Danilo e toda minha família que sempre me apoiaram e incentivaram; aos velhos e novos amigos, pelo apoio e companherismo, em especial as amigas Andréa, Luciene e Silvia e ao amigo Miguel; a todos meus professores da escola, da UNESP e da USP, pelos conhecimentos transmitidos; aos funcionários do ICMC e a todos que direta ou indiretamente contribuíram para o andamento e conclusão deste trabalho. 


\section{Resumo}

Neste trabalho apresentamos generalizações dos resultados sobre ideais primários em anéis de Witt contidos em [05], para anéis de Witt de anéis semilocais. 


\begin{abstract}
In this work we generalize the results about primary ideals in Witt rings contained in [05], for Witt rings of semilocal rings.
\end{abstract}




\section{Índice}

Introdução 1

1 Preliminares 3

2 Espaços Bilineares 12

2.1 Definições .......................... 12

2.2 Extensão de escalares . . . . . . . . . . . . . . . . . . 14

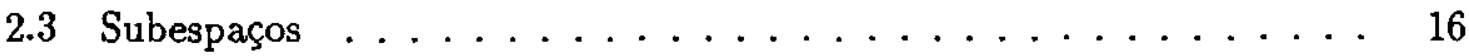

2.4 Espaços Metabólicos e Hiperbólicos . . . . . . . . . . . . . 23

3 O Anel de Witt 30

3.1 Geradores de $\mathcal{W}(A) \ldots \ldots \ldots . \ldots . \ldots 30$

3.2 Os ideais primos de $\mathcal{W}(A) \ldots \ldots \ldots . \ldots \ldots$

$3.3 \operatorname{Nil}(\mathcal{W}(A))$ e $\mathcal{W}_{t}(A) \ldots \ldots \ldots \ldots \ldots$

4 Ideais Primários no Anel de Witt 46

4.1 Ideais Primários de $\mathcal{W}(A) \ldots \ldots \ldots 46$

4.2 Decomposição Primária em $\mathcal{W}(A) \ldots \ldots \ldots$. . . . . . . 51

4.3 Ideais contendo uma forma de dimensão ímpar . . . . . . . . . . 58

Referências Bibliográficas $\quad 67$ 


\section{Introdução}

Desde seu nascimento (provavelmente na Babilonia) até 1936 o estudo de formas quadráticas era feito com formas sobre o corpo dos números reais, o corpo dos números complexos ou o anel dos inteiros. A fundamentação da teoria das formas quadráticas sobre um corpo genérico apareceu em um trabalho de Ernst Witt, em 1937, onde ele teve a brilhante idéia de considerar não só o estudo de uma forma quadrática em particular, mas sim o conjunto de todas as formas quadráticas sobre um corpo genérico de característica distinta de 2. Este conjunto, ele repartiu em classes de equivalências e construiu um objeto algébrico - o anel de Witt - que tornou-se o principal objeto de toda a teoria. Mas, demorou 30 anos para que fosse demonstrada a importância das idéias de Witt por Albrecht Pfister com seus teoremas de estruturas, criando assim a teoria "algébrica"das formas quadráticas. A partir daí, todo o estudo de classificação de formas quadráticas se resume ao estudo da estrutura do anel de Witt.

Em [08] e [09], Knebusch, Rosenberg e Ware apresentam resultados sobre a estrutura de anéis de Witt vistos como quocientes de anéis de grupos abelianos. Com tais resultados, obtemos a classificação dos ideais primos do anel de Witt das formas bilineares sobre um anel semilocal.

Em [05] Robert W. Fitzgerald apresenta, um estudo sobre os ideais primários do anel de Witt dos espaços bilineares sobre um corpo de característica distinta de 2. Usando as técnicas utilizadas em [08], [09] e também em [04], neste trabalho apresentamos o desenvolvimento de Fitzgerald para anéis de Witt dos espaços bilineares sobre um anel semilocal sem impormos condições sobre 2 ser ou não inversível em 
tais anéis.

Para tanto, nos capítulos I e II, apresentamos alguns resultados de álgebra comutativa e noções básicas sobre espaços bilineares sobre anéis semilocais necessários à compreensão do restante do trabalho.

O capítulo III contém resultados sobre a estrutura do anel de Witt, $\mathcal{W}(A)$, dos espaços bilineares sobre um anel semilocal $A$. Mais precisamente, apresentamos a caracterização dos geradores de $\mathcal{W}(A)$ e dos ideais primos de $\mathcal{W}(A)$. Alguns resultados sobre assinaturas, os elementos nilpotentes e os elementos de torção de $\mathcal{W}(A)$ são também apresentados neste capítulo.

O capítulo IV, consiste do que nos propomos a desenvolver no projeto, ou seja, as generalizações dos resultados de Fitzgerald para o anel de Witt dos espaços bilineares sobre um anel semilocal $A$. Na primeira seção, usando a caracterização dos ideais primos de $\mathcal{W}(A)$, apresentamos a caracterização dos ideais $\mathcal{P}$-primários de $\mathcal{W}(A)$, para cada tipo de ideal primo $\mathcal{P}$ de $\mathcal{W}(A)$. A seção 2 contém resultados sobre decomposição primária em $\mathcal{W}(A)$ e, o principal deles, apresenta condições necessárias e suficientes sobre o anel semilocal $A$ para que todo ideal de $\mathcal{W}(A)$ seja decomponível. Finalmente, na última seção apresentamos alguns resultados sobre os ideais de $\mathcal{W}(A)$ que não estão contidos no ideal fundamental $\mathfrak{I}(A)$, ou seja, sobre os ideais que contém formas de dimensão ímpar. 


\section{Capítulo 1}

\section{Preliminares}

Neste capítulo apresentaremos alguns fatos básicos de álgebra comutativa, bem como a introdução e algumas propriedades dos ideais primários, necessários para o desenvolvimento deste trabalho. Algumas demonstrações serão omitidas, as quais podem ser encontradas na literatura, como por exemplo em [01]. No que segue e nos demais capítulos, $A$ denotará sempre um anel comutativo com elemento identidade 1 . Indicaremos por $\operatorname{Spec}(A)$ o conjunto dos ideais primos de $A$, por $\operatorname{Spm}(A)$ o conjunto dos ideais maximais de $A$, por $\mathcal{\partial}(A)$ o radical de Jacobson de $A$ e por $A^{*}$ o grupo das unidades de $A$. Assumiremos, também que todo $A$-módulo será unitário e que todo homomorfismo de aneis leva elemento identidade em elemento identidade.

Os próximos resultados serão usados frequentemente no decorrer deste trabalho. O primeiro deles caracteriza $\partial(A)$.

Proposição 1.1 Um elemento $x \in A$ está em $\partial(A)$ se, e somente se $1-x y \in A^{*}$, para todo $y \in A$.

Dem.: Ver (1.9) de [01].

Proposição 1.2 Sejam $\mathfrak{I}$ um ideal de $A$ contido em $\mathcal{J}(A)$ e $a \in A$. Então $\bar{a}=a+\mathfrak{I}$ é uma unidade em $A / \mathfrak{I}$ se, e somente se a é uma unidade em $A$. 
Dem.: Se $a$ é uma unidade em $A$, então é imediato que $a+\mathfrak{I}$ é inversível em $A / \mathfrak{I}$. Reciprocamente, se $a+\mathfrak{I} \in(A / \mathfrak{I})^{*}$, então existe $b \in A$ tal que $(a+\mathfrak{I})(b+\mathfrak{I})=1+\mathfrak{I}$, ou seja, $1-a b \in \mathfrak{I} \subseteq \mathcal{J}(A)$. De (1.1) temos $a b \in A^{*}$ e, consequentemente $a \in A^{*}$.

Para $\mathfrak{M} \in \operatorname{Spm}(A)$, denotaremos por $A_{\mathfrak{M}}$ a localização de $A$ em $\mathfrak{M}$.

Proposição 1.3 Seja $a \in A$. São equivalentes:

(i) $a \in A^{*}$;

(ii) $\frac{a}{1} \in\left(A_{\mathfrak{M}}\right)^{*}$, para todo $\mathfrak{M} \in \operatorname{Spm}(A)$;

(iii) $\bar{a} \neq \overrightarrow{0}$ em $(A / \mathfrak{M})^{*}$, para todo $\mathfrak{M} \in \operatorname{Spm}(A)$.

Dem.: Imediata.

Definição 1.4 Dizemos que um anel comutativo $A$, com elemento identidade 1 é um anel semilocal se $\operatorname{Spm}(A)$ é finito, ou seja, se $A$ tem somente um número finito de ideais maximais.

Exemplo 1.5 Todo corpo é um anel semilocal, ou mais geralmente, todo produto direto finito de corpos é um anel semilocal. Da correspondência entre os ideais de $A \mathrm{e}$ os ideais do anel quociente, temos que toda imagem homomórfica de um anel semilocal é também semilocal. Mais ainda, se $A$ é um anel semilocal, então $A_{\mathcal{P}}$ também o é, para todo $\mathcal{P} \in \operatorname{Spec}(A)$.

Proposição 1.6 Sejam A um anel semilocal com $\operatorname{Spm}(A)=\left\{\mathfrak{M}_{1}, \ldots, \mathfrak{M}_{r}\right\}$. Então

$$
\frac{A}{\partial(A)} \cong \frac{A}{\mathfrak{M}_{1}} \times \ldots \times \frac{A}{\mathfrak{M}_{r}},
$$

onde $\cong$ denota isomorfismo de anéis. 
Dem.: Segue diretamente de (1.10) de [01].

Como, neste trabalho, apresentaremos um estudo dos ideais primários do anel de Witt dos espaços bilineares sobre um anel semilocal, achamos conveniente recordar - conceito e alguns resultados básicos sobre ideais primários de um anel comutativo. Tais resultados serão apresentados sem demonstrações as quais podem ser encontradas, por exemplo, em [01].

Definição 1.7 Um ideal $\mathfrak{I}$ do anel $A$ é dito ser um ideal primário se $\mathfrak{I} \neq A$ e se $x y \in \mathfrak{I}$, então $x \in \mathfrak{I}$ ou $y^{n} \in \mathfrak{I}$, para algum $n>0$. Em outras palavras, $\mathfrak{I}$ é um ideal primário de $A$ se, e somente se $A / \mathfrak{I} \neq 0$ e todo divisor de zero em $A / \mathfrak{I}$ é nilpotente.

Seja $\mathfrak{I}$ um ideal primário de $A$ e $r(\mathfrak{I})=\left\{x \in A ; x^{n} \in \mathfrak{I}\right.$, para algum $\left.n \geq 1\right\} \circ$ radical de $\mathfrak{I}$, o qual é um ideal primo. Se $r(\mathfrak{I})=\mathcal{P} \in \operatorname{Spec}(A)$, dizemos que $\mathfrak{I}$ é um ideal $\mathcal{P}$-primário. Recordemos também que dados $\mathfrak{I}$ e $\mathfrak{H}$ ideais de $A$, o ideal quociente de $\mathfrak{I}$ por $\mathcal{H}$ é o ideal de $A,(\mathfrak{I}: \mathcal{H})=\{x \in A ; x \mathcal{H} \subseteq \mathfrak{I}\}$.

Mais ainda, um ideal $\mathfrak{I}$ do anel $A$ é dito ser decomponivel se $\mathfrak{I}$ admite uma decomposição como uma intersecção finita de ideais primários. Todo ideal decomponível $\mathfrak{I}$ admite uma decomposição primária reduzida, ou seja,

$$
\mathfrak{I}=Q_{1} \cap Q_{2} \cap \ldots \cap Q_{n},
$$

onde os ideais $\left\{Q_{1}, \ldots, Q_{n}\right\}$ satisfazem:

(i) $Q_{i}$ é primário, para cada $i=1, \ldots, n$;

(ii) $r\left(Q_{i}\right) \neq r\left(Q_{j}\right)$, se $i \neq j, 1 \leq i, j, \leq n$;

(iii) $\bigcap_{j \neq i} \Omega_{j} \nsubseteq \Omega_{i}$, para cada $i=1, \ldots, n$.

Para cada ideal $\mathfrak{I}$ de $A$, um ideal primo $\mathcal{P}$ é dito ser um divisor primo associado de $\mathfrak{I}$ se $(\mathfrak{I}: A x)$ é $\mathcal{P}$-primário, para algum $x \in A$. Denotamos por Assoc $(\mathfrak{I})$ o conjunto dos divisores primos associados de $\mathfrak{I}$. 
Sobre a unicidade de decomposições primárias de ideais temos os seguintes resultados gerais:

Teorema 1.8 Sejam $\mathfrak{I}$ um ideal decomponivel de $A$ e $\bigcap_{i=1}^{n} Q_{i}$ uma decomposição primária reduzida de $\mathfrak{I}$. Se $\mathcal{P}_{i}=r\left(Q_{i}\right), 1 \leq i \leq n$, então estes são precisamente os ideais primos que aparecem no conjunto de ideais $\{r(\mathfrak{I}: A x) ; x \in A\}$ e, portanto, são independentes da particular decomposição de $\mathfrak{I}$.

Dem.: Ver (4.5) de [01].

Um conjunto $\sum \subseteq$ Assoc $(\mathfrak{I})$ é dito ser um conjunto isolado, se $\sum$ satisfaz a seguinte condição: Se $\mathcal{P}$ é um ideal primo associado com $\mathfrak{I}$ e $\mathcal{P}^{\prime} \subseteq \mathcal{P}$ para algum $\mathcal{P} \in \sum$, então $\mathcal{P}^{\prime} \in \sum$.

Como um segundo teorema de unicidade de decomposições primárias temos:

Teorema 1.9 Sejam $\mathfrak{I}$ um ideal decomponível de $A, e \bigcap_{i=1}^{n} \mathcal{Q}_{i}$ uma decomposição primária reduzida de $\mathfrak{I}, e\left\{\mathcal{P}_{i_{1}}, \ldots, \mathcal{P}_{i_{m}}\right\} \subseteq$ Assoc $(\mathfrak{I})$ um conjunto isolado. Então $\Omega_{i_{1}} \cap \ldots \cap \Omega_{i_{m}}$ é independente da decomposição.

Dem.: Ver (4.10) de [01].

Sejam $A$ um anel e $M$ um $A$-módulo. Para um ideal I de $A$, denotamos por $M(\mathfrak{I})$ o $\frac{A}{\mathfrak{I}}$-módulo $M \otimes\left(\frac{A}{\mathfrak{I}}\right) \cong \frac{M}{\mathfrak{I} M}$, onde $\otimes$ denotará sempre $\otimes_{A}$. Para $\mathcal{P} \in \operatorname{Spec}(A)$,

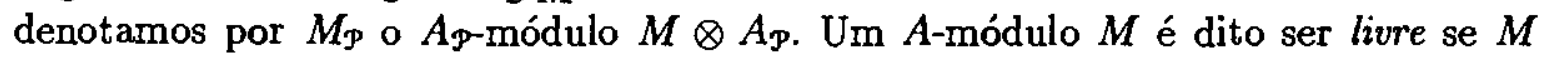
admite uma base, ou seja se existe um conjunto $\left\{x_{i} ; i \in \Gamma\right\}$ de elementos de $M$, tais que $M \cong \bigoplus_{i \in \Gamma} A x_{i}$. Se, além disso, $\Gamma$ é finito com $m$ elementos, dizemos que $M$ é livre de dimensão $m$ e escrevemos $\operatorname{dim}(M)=m$. 
Proposição 1.10 Seja $A=F_{1} \times \ldots \times F_{r}$ um produto direto finito de corpos. Se $M$ é um A-módulo livre de dimensão $m$, então $M \cong M_{1} \times \ldots \times M_{r}$ onde cada $M_{i}$ é um $F_{i}$-espaço vetorial de dimensão $m$, para $i=1, \ldots, r$.

Dem.: Segue diretamente do fato que o produto cartesiano comuta com a soma direta.

Proposição 1.11 Sejam $A$ um anel semilocal, $\mathfrak{I}$ um ideal de $A$ com $\mathfrak{I} \subseteq \mathcal{J}(A)$ e $M$ um A-módulo. Se $\left\{x_{1}, \ldots, x_{n}\right\} \subseteq M$ são tais que $\left\{\overline{x_{1}}, \ldots, \overline{x_{n}}\right\}$ é uma base de $M(\mathfrak{I})$ sobre $A / \mathfrak{I}$, então $\left\{x_{1}, \ldots, x_{n}\right\}$ é uma base de $M$ sobre $A$.

Dem.: Seja $N=A x_{1}+\ldots+A x_{n} \subseteq M$. Desde que $\left\{\overline{x_{1}}, \ldots, \overline{x_{n}}\right\}$ é uma base de $M(\mathfrak{I})$ sobre $A / \mathfrak{I}$ e $N(\mathfrak{I})=(A / \mathfrak{I}) \overline{x_{1}}+\ldots+(A / \mathfrak{I}) \overline{x_{n}}$, temos que $M(\mathfrak{I})=N(\mathfrak{I})$, ou seja, $M / \mathfrak{I} M=N / \mathfrak{I} N$. Assim $M=N+\mathfrak{I} M$ e, desde que $\mathfrak{I} \subseteq \mathfrak{J}(A)$, do Lema de Nakayama temos que $M=N$, ou seja $\left\{x_{1}, \ldots, x_{n}\right\}$ é um conjunto de geradores de $M$.

Se existe $i=1, \ldots, n$ tal que $x_{i} \in A x_{1}+\ldots+A x_{i-1}+A x_{i+1}+\ldots+A x_{n}$, então $\overline{x_{i}} \in(A / \mathfrak{I}) \overline{x_{1}}+\ldots+(A / \mathfrak{I}) \overline{x_{i-1}}+(A / \mathfrak{I}) \overline{x_{i+1}}+\ldots+(A / \mathfrak{I}) \overline{x_{n}}$, o que contradiz o fato de $\left\{\overline{x_{1}}, \ldots, \overline{x_{n}}\right\}$ ser uma base de $M(\mathfrak{I})$. Consequentemente, $\left\{x_{1}, \ldots, x_{n}\right\}$ é uma base de $M$ sobre $A$, como queríamos.

Seja $A$ um anel. Uma seqüência de $A$-módulos e $A$-homomorfismos

$$
\cdots \longrightarrow M_{i-1} \stackrel{f_{i}}{\longrightarrow} M_{i} \stackrel{f_{i+1}}{\longrightarrow} M_{i+1} \longrightarrow \cdots
$$

é dita ser exata em $M_{i}$ se $\operatorname{Im}\left(f_{i}\right)=\operatorname{Ker}\left(f_{i+1}\right)$. Se a seqüência é exata em cada $M_{i}$, então dizemos que ela é uma seqüência exata.

Uma seqüência exata do tipo $0 \longrightarrow M_{1} \stackrel{f}{\longrightarrow} M_{2} \stackrel{g}{\longrightarrow} M_{3} \longrightarrow 0$, é chamada uma seqüência exata curta. 
Dizemos que uma seqüência exata

$$
\cdots \longrightarrow M_{i-1} \stackrel{f_{i}}{\rightarrow} M_{i} \stackrel{f_{i+1}}{\longrightarrow} M_{i+1} \longrightarrow \cdots
$$

cinde em $M_{i}$, se o submódulo $X=\operatorname{Im}\left(f_{i}\right)=\operatorname{Ker}\left(f_{i+1}\right)$ é um somando direto de $M_{i}$. No caso de uma seqüência exata curta $0 \rightarrow X \rightarrow Y \rightarrow Z \rightarrow 0$, obviamente temos que ela cinde em $X$ e $Z$. Se, além disso, a seqüência cinde em $Y$, dizemos apenas que a seqüência exata cinde.

Proposição 1.12 Se uma seqüência exata curta de A-módulos

$$
0 \longrightarrow X \stackrel{f}{\longrightarrow} Y \stackrel{g}{\longrightarrow} Z \longrightarrow 0
$$

cinde, então $Y$ é isomorfo, como A-módulo, à $X \oplus Z$.

Dem.: Desde que a seqüência exata $0 \longrightarrow X \stackrel{f}{\rightarrow} Y \stackrel{g}{\rightarrow} Z \longrightarrow 0$ cinde, temos que $X$ é um somando direto de $Y$, ou seja $Y=X \oplus W$, para algum $A$-módulo $W$. Temos, também que $X=\operatorname{Im}(f)=\operatorname{Ker}(g)$. Assim, desde que $g$ é sobrejetor, temos

$$
\frac{Y}{\operatorname{Ker}(g)} \cong \operatorname{Im}(g)=Z \text {. }
$$

Portanto, $Z$ é isomorfo ao complementar de $X$ em $Y$, ou seja, $Z \cong W$ como $A$-módulos. Assim $Y=X \oplus W \cong X \oplus Z$, como queríamos.

Um $A$-módulo $P$ é dito ser um $A$-módulo projetivo se satisfaz uma das seguintes condições equivalentes:

(a) $P$ é somando direto de um $A$-módulo livre.

(b) Toda seqüência exata curta de $A$-módulos $0 \longrightarrow M \longrightarrow N \longrightarrow P \longrightarrow 0$, cinde.

(c) Para toda seqüência exata curta de $A$-módulos $0 \longrightarrow X \longrightarrow Y \longrightarrow Z \longrightarrow 0$, a seqüência de $A$-módulos

$$
0 \longrightarrow \operatorname{Hom}(P, X) \longrightarrow \operatorname{Hom}(P, Y) \longrightarrow \operatorname{Hom}(P, Z) \longrightarrow 0
$$

é exata. 
(d) Para todo diagrama de $A$-módulos e $A$-homomorfismos

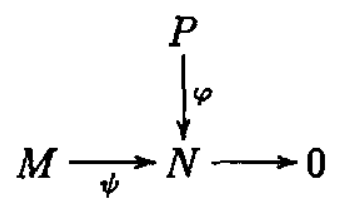

existe um levantamento de $\varphi, \varphi^{*}: P \rightarrow M$, tal que o diagrama abaixo é comutativo, isto é, $\psi \circ \varphi^{*}=\varphi$.

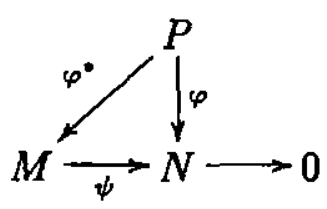

Não apresentaremos aqui a demonstração das equivalências que caracterizam um $A$-módulo projetivo, pois fojem dos objetivos de nosso trabalho mas, esta caracterização é clássica e pode ser encontrada, por exemplo, em [12].

É imediato que todo $A$-módulo livre é também projetivo.

Se $M$ é um $A$-módulo projetivo finitamente gerado, e $\mathfrak{M} \in \operatorname{Spm}(A)$, definimos o posto de $M$ módulo $\mathfrak{M}$, como sendo a dimensão do $(A / \mathfrak{M})$-espaço vetorial $M(\mathfrak{M})$. Dizemos que $M$ é um $A$-módulo projetivo finitamente gerado de posto constante se a aplicação $\rho: \operatorname{Spm}(A) \longrightarrow \mathbb{Z}$, definida por $\rho(\mathfrak{M})=\operatorname{dim}_{A /{ }_{\mathfrak{N}}} M(\mathfrak{M})$ é constante.

A teoria algébrica das formas bilineares sobre anéis é desenvolvida na categoria dos módulos projetivos finitamente gerados e de posto constante. Finalizaremos este capítulo mostrando que, sobre anéis semilocais, esta categoria coincide com a categoria dos módulos livres de dimensão finita. Para tanto, usaremos o seguinte resultado auxiliar.

Lema 1.13 Sejam $P$ um A-módulo projetivo finitamente gerado e $\mathfrak{I} \subseteq \mathcal{J}(A)$ um ideal de $A$. Se $P(\mathfrak{I})$ é um $(A / \mathfrak{I})$-módulo livre, então $P$ é um A-módulo livre. 
Dem.: Desde que $P(\mathfrak{I})$ é um $(A / \mathfrak{I})$-módulo livre e $P$ é finitamente gerado, então existe $m \in \mathbb{N}$ tal que $P(\mathfrak{I}) \cong(A / \mathfrak{I})^{m}$. Considerando as sobrejeções naturais de $P$ em $P(\mathfrak{I})$ e de $A^{m}$ em $(A / \mathfrak{I})^{m}$, temos o seguinte diagrama de $A$-módulos.

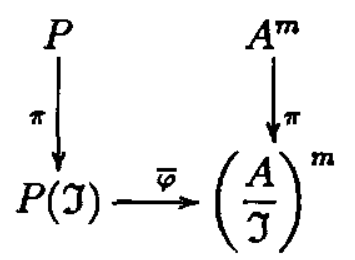

onde $\pi$ denota as sobrejeções naturais e $\bar{\varphi}$ é um isomorfismo de $(A / \mathfrak{I})$-módulos e, portanto, de $A$-módulos. Temos então o diagrama

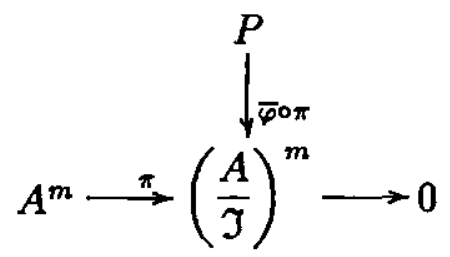

e, desde que $P$ é projetivo, temos que existe $\varphi: P \longrightarrow A^{m}$ tal que o diagrama abaixo é comutativo

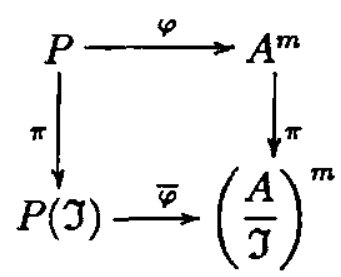

Agora, é suficiente mostrarmos que $\varphi$ é um isomorfismo. Desde que $\pi \circ \varphi=\bar{\varphi} \circ \pi$ é sobrejetor, temos que $\pi(\operatorname{Im}(\varphi))=(A / \mathfrak{I})^{m}$, ou seja, $\operatorname{Im}(\varphi)+\mathfrak{I} A^{m}=A^{m}$. Como $\mathfrak{I} \subseteq \mathcal{J}(A)$, do Lema de Nakayama temos que $\operatorname{Im}(\varphi)=A^{m}$, isto é, $\varphi$ é sobrejetor. Logo temos a seqüência exata $0 \longrightarrow \operatorname{Ker}(\varphi) \longrightarrow P \longrightarrow A^{m} \longrightarrow 0$. Mas, $A^{m}$ é um $A$-módulo livre e, portanto projetivo, o que implica que $P \cong \operatorname{Ker}(\varphi) \oplus A^{m}$. Assim $\operatorname{Ker}(\varphi)$ é um $A$-módulo finitamente gerado tal que $\frac{\operatorname{Ker}(\varphi)}{\mathfrak{I} \operatorname{Ker}(\varphi)}=\operatorname{Ker}(\bar{\varphi})=\{0\}$, pois $\bar{\varphi}$ é injetor.

Portanto $\operatorname{Ker}(\varphi) \cong \mathfrak{I} \operatorname{Ker}(\varphi)$ e, novamente pelo Lema de Nakayama, temos que $\operatorname{Ker}(\varphi)=\{0\}$, o que completa a demonstração. 
No que segue, exceto menção em contrário, $A$ denotará um anel semilocal, $\operatorname{Spm}(A)=\left\{\mathfrak{M}_{1}, \ldots, \mathfrak{M}_{r}\right\}$ o conjunto do todos os ideais maximais de $A$, e todos os módulos considerados serão $A$-módulos.

Teorema 1.14 Todo módulo projetivo finitamente gerado e de posto constante sobre um anel semilocal é livre.

Dem.: Seja $P$ um módulo projetivo finitamente gerado e de posto constante sobre $A$, com $n=\rho(P)$, ou seja, $n=\operatorname{dim}_{A / \mathfrak{M}_{i}}\left(P\left(\mathfrak{M}_{i}\right)\right)$, para qualquer $\mathfrak{M}_{i} \in \operatorname{Spm}(A)$. Para $\bar{P}=\frac{P}{\mathcal{J}(A) P}$, temos

$$
\bar{P} \cong \frac{P}{\mathfrak{M}_{1} P} \oplus \ldots \oplus \frac{P}{\mathfrak{M}_{r} P} \cong\left(\frac{A}{\mathfrak{M}_{1}}\right)^{n} \oplus \ldots \oplus\left(\frac{A}{\mathfrak{M}_{r}}\right)^{n} \cong\left(\frac{A}{\mathfrak{M}_{1}} \times \ldots \times \frac{A}{\mathfrak{M}_{r}}\right)^{n} .
$$

Logo $\bar{P}$ é um $\bar{A}=(A / \mathcal{J}(A))$-módulo livre de dimensão $n$ e, do lema anterior, segue que $P$ é um $A$-módulo livre. 


\section{Capítulo 2}

\section{Espaços Bilineares}

Neste capítulo apresentaremos a noção e alguns resultados básicos da teoria das formas bilineares sobre um anel semilocal $A$. Alguns destes resultados valem mais geral para um anel comutativo com elemento identidade.

Em geral, a teoria das formas bilineares sobre um anel $A$, é feita na categoria dos $A$-módulos projetivos finitamente gerados e de posto constante. Como os principais resultados apresentados neste trabalho são sobre espaços bilineares sobre aneis semilocais e, de (1.14) temos que, neste caso, todo módulo projetivo finitamente gerado e de posto constante é livre, por conveniência de redação, trabalharemos desde o início com a categoria dos $A$-módulos livres de dimensão finita. Denotaremos tal categoria por $\mathcal{L}(A)$. Exceto quando mencionado o contrário, $\otimes$ significará sempre $\otimes_{A}$. Para cada $M \in \mathcal{L}(A)$, denotaremos por $M^{*} \circ A$-módulo dual $\operatorname{Hom}_{A}(M, A) \in \mathcal{L}(A)$.

\subsection{Definições}

Definição 2.1 $O$ par $(M, b)$ consistindo de um módulo $M \in \mathcal{L}(A)$ e de uma forma bilinear simétrica $b: M \times M \rightarrow A$ é dito ser um módulo bilinear sobre $A .0$ módulo bilinear $(M, b)$ é não singular, ou simplesmente e um espaço bilinear, se a função $A$-linear $d_{b}: M \rightarrow M^{*}$, definida por $d_{b}(x)=b(x, \quad)$, para todo $x \in M$, é um isomor- 
fismo de $A$-módulos. A função $d_{b}$ é chamada a adjunta da forma bilinear $b$. Se o $A$-módulo $M$ tem dimensão $n$, dizemos que o espaço bilinear $(M, b)$ tem dimensão $n$ e indicamos por $\operatorname{dim}(M, b)=n$, ou simplesmente por $\operatorname{dim}(M)=n$, ou ainda $\operatorname{dim}(b)=n$. Uma isometria entre dois módulos bilineares $\left(M_{1}, b_{1}\right)$ e $\left(M_{2}, b_{2}\right)$ é um isomorfismo de $A$-módulos $\varphi: M_{1} \rightarrow M_{2}$, que preserva a forma bilinear, ou seja, $b_{1}(x, y)=b_{2}(\varphi(x), \varphi(y))$, para todo $x, y \in M_{1}$. Quando existe uma isometria entre $\left(M_{1}, b_{1}\right)$ e $\left(M_{2}, b_{2}\right)$ dizemos que os módulos bilineares são isométricos e denotamos por $\left(M_{1}, b_{1}\right) \simeq\left(M_{2}, b_{2}\right)$, ou simplesmente $b_{1} \simeq b_{2}$, ou ainda $M_{1} \simeq M_{2}$.

Se $(M, b)$ é um módulo bilinear sobre $A$ e $\left\{x_{1}, \cdots, x_{n}\right\}$ é uma base de $M$, então a forma bilinear $b$ é determinada pela matriz quadrada $\left(b_{i j}\right)=\left(b\left(x_{i}, x_{j}\right)\right), 1 \leq i, j \leq n$, pois para $x=\sum_{i=1}^{n} \alpha_{i} x_{i}$ e $y=\sum_{j=1}^{n} \beta_{j} x_{j}$ em $M$, temos $b(x, y)=\sum_{i, j=1}^{n} b_{i j} \alpha_{i} \beta_{j}$. Reciprocamente, para cada matriz simétrica $n \times n,\left(b_{i j}\right)$ sobre $A$ obtemos uma forma bilinear simétrica sobre $M$ dada pela mesma fórmula descrita acima e, o módulo bilinear $(M, b)$ é não singular se, e somente se $\operatorname{det}\left(b_{i j}\right)$ é uma unidade em $A$.

Chamaremos de determinante do espaço bilinear $b$ e denotaremos por $\operatorname{det}(b)$ o determinante da matriz $\left(b_{i j}\right)$. No que segue, identificaremos um elemento $x \in M$, com o vetor das coordenadas de $x$ em relação a uma dada base de $M$. Mais ainda, como um módulo $(M, b)$ é caracterizado por uma matriz quadrada $\left(b_{i j}\right)$, usaremos também a notação $b=\left(b_{i j}\right)$ para indicarmos a forma bilinear $b$.

Denotamos a categoria dos espaços bilineares sobre $A$ por $\operatorname{Bil}(A)$, onde os morfismos desta categoria são as isometrias. $\operatorname{Em} \operatorname{Bil}(A)$ definimos duas operações, uma soma e um produto.

Definição 2.2 Definimos a soma ortogonal dos espaços $\left(M_{i}, b_{i}\right) \in \mathcal{B i l}(A)$, como sendo o módulo bilinear

$$
\left(M_{1}, b_{1}\right) \perp\left(M_{2}, b_{2}\right)=\left(M_{1} \oplus M_{2}, b_{1} \perp b_{2}\right)
$$

onde $\left(b_{1} \perp b_{2}\right)\left(x_{1}+x_{2}, y_{1}+y_{2}\right)=b_{1}\left(x_{1}, y_{1}\right)+b_{2}\left(x_{2}, y_{2}\right)$, para todo $x_{;}, y_{i} \in M_{i}, i=1,2$. 
É fácil ver que $\left(M_{1} \oplus M_{2}, b_{1} \perp b_{2}\right)$ é de fato um espaço bilinear sobre $A$. Denotamos este espaço simplesmente por $b_{1} \perp b_{2}$, ou ainda $M_{1} \perp M_{2}$.

Definição 2.3 Definimos o produto tensorial dos espaços $\left(M_{i}, b_{i}\right) \in \mathcal{B} i l(A), i=1,2$, como sendo o módulo bilinear

$$
\left(M_{1}, b_{1}\right) \otimes\left(M_{2}, b_{2}\right)=\left(M_{1} \otimes M_{2}, b_{1} \otimes b_{2}\right),
$$

onde $\left(b_{1} \otimes b_{2}\right)\left(x_{1} \otimes x_{2}, y_{1} \otimes y_{2}\right)=b_{1}\left(x_{1}, y_{1}\right) b_{2}\left(x_{2}, y_{2}\right)$, para todo $x_{i}, y_{i} \in M_{i}$. Novamente, pode-se ver que este módulo é não singular, ou seja, é de fato um espaço bilinear sobre $A$. Denotamos este espaço por $b_{1} \otimes b_{2}$.

Mostra-se facilmente que essas duas operações são associativas, comutativas e o produto tensorial é distributivo em relação a soma ortogonal. Além disso, as duas operações são compatíveis com a relação de isometria, ou seja, se $b_{1} \simeq b_{2}$ e $b_{1}^{\prime} \simeq b_{2}^{\prime}$, então

$$
b_{1} \perp b_{1}^{\prime} \simeq b_{2} \perp b_{2}^{\prime} \quad \text { e } \quad b_{1} \otimes b_{1}^{\prime} \simeq b_{2} \otimes b_{2}^{\prime} .
$$

Exemplo 2.4 Sejam $M=A x$ e $\alpha \in A$. A forma bilinear $b: M \times M \rightarrow A$ definida por $b(\gamma x, \beta x)=\gamma \beta b(x, x)=\gamma \beta \alpha$, para todo $\gamma, \beta \in A$, é não singular se, e somente se $\alpha \in A^{\star}$. Denotaremos esta forma bilinear, simplesmente por $\langle\alpha\rangle$. Mais geralmente, a forma bilinear $b=\left\langle\alpha_{1}\right\rangle \perp \ldots \perp\left\langle\alpha_{n}\right\rangle$ será denotada por $b=\left\langle\alpha_{1}, \ldots, \alpha_{n}\right\rangle$ e, neste caso, $b$ é não singular se, e somente se $\alpha_{1} \alpha_{2} \ldots \alpha_{n} \in A^{*}$.

Sejam $M=A x \oplus A y$ e $\alpha, \beta \in A$. Definimos uma forma $A$-bilinear $b: M \times M \rightarrow A$ por $b(x, x)=\alpha, b(y, y)=\beta$ e $b(x, y)=1$. Esta forma bilinear é denotada por $b=\left(\begin{array}{cc}\alpha & 1 \\ 1 & \beta\end{array}\right)$ e, $b$ é não singular se, e somente se $1-\alpha \beta \in A^{*}$.

\subsection{Extensão de escalares}

Sejam $\varphi: A \longrightarrow A^{\prime}$ um homomorfismo de anéis e $(M, b) \in \mathcal{B} i l(A)$. É facil ver que se $\left\{x_{1}, x_{2}, \ldots, x_{n}\right\}$ é uma base de $M$ sobre $A$, então $\left\{x_{1} \otimes 1, x_{2} \otimes 1, \ldots, x_{n} \otimes 1\right\}$ é 
uma base de $M \otimes A^{\prime}$ sobre $A^{\prime}$, ou seja, $M \otimes A^{\prime} \in \mathcal{L}\left(A^{\prime}\right)$. Sobre tal $A^{\prime}$-módulo difinimos uma forma bilinear simétrica $b^{\prime}=b \otimes A^{\prime}$ por $b^{\prime}(x \otimes \alpha, y \otimes \beta)=\varphi(b(x, y)) \alpha \beta$ para todo $x, y \in M$ e $\alpha, \beta \in A$. Agora, se $\left(b_{i j}\right)=\left(b\left(x_{i}, x_{j}\right)\right)$ é a matriz associada ao espaço bilinear $(M, b)$, então, em relação à base $\left\{x_{1} \otimes 1, \ldots, x_{n} \otimes 1\right\}$ de $M \otimes A^{\prime}$, o módulo bilinear $\left(M \otimes A^{\prime}, b^{\prime}\right)$ tem a matriz associada $\left(b_{i j}^{\prime}\right)=\left(b^{\prime}\left(x_{i} \otimes 1, x_{j} \otimes 1\right)\right)=\left(b\left(x_{i}, x_{j}\right)\right)=$ $\left(b_{i j}\right)$, o que mostra que $b^{\prime}$ é também não singular. O espaço bilizear $\left(M \otimes A^{\prime}, b \otimes A^{\prime}\right)$ de $\operatorname{Bil}\left(A^{\prime}\right)$ é dito ser o espaço obtido de $(M, b)$ por estensão de escalares.

Em particular, para $\mathfrak{M}$ um ideal maximal de $A$, seja $\varphi: A \longrightarrow A_{\mathfrak{g}}$ o homomorfismo canônico $\varphi(\alpha)=\frac{\alpha}{1}$, para todo $\alpha \in A$. Dado $(M, b) \in \mathcal{B} i l(A)$, temos que $M \otimes A_{\mathfrak{M}} \cong M_{\mathfrak{M}} \mathrm{e}, b_{\mathfrak{M} \pi}=b \otimes A_{\mathfrak{M}}$ é dada por $b_{\mathfrak{M} \pi}\left(\frac{x}{\alpha}, \frac{y}{\beta}\right)=\frac{b(x, y)}{\alpha \beta}$, para todo $x, y \in M$ e $\quad \alpha, \beta \in A-\mathfrak{M}$. O espaço bilinear $\left(M_{\mathfrak{M}}, b_{\mathfrak{M}}\right)$ é chamado a localização de $(M, b)$ em $\mathfrak{M}$.

Para um ideal $\mathfrak{I}$ qualquer de $A$, considerando a projeção canônica $\varphi: A \longrightarrow A / \mathfrak{I}$, temos que para todo espaço bilinear $(M, b) \in \mathcal{B i l}(A) \quad \varphi^{*}(M, b)=(M(\mathfrak{I}), b(\mathfrak{I}))$ é um elemento de $\operatorname{Bil}(A / \mathfrak{I})$, onde $M(\mathfrak{I})=M \otimes(A / \mathfrak{I}) \cong M / \mathfrak{I} M$ e $b(\mathfrak{I})(\bar{x}, \bar{y})=$ $\varphi(b(x, y))=\overline{b(x, y)}$, para todo $\bar{x}, \bar{y} \in M / \mathfrak{I} M$. O espaço $(M(\mathfrak{I}), b(\mathfrak{I}))$ é chamado a redução módulo $\mathfrak{I}$ de $(M, b)$.

Com esta noção de redução e localização para módulos bilineares temos

Proposição 2.5 Seja $(M, b)$ um módulo bilinear sobre A. São equivalentes:

(i) $(M, b)$ é não singular;

(ii) $\left(M_{\mathfrak{N}}, b_{\mathfrak{M}}\right)$ é não singular, para todo $\mathfrak{M} \in \operatorname{Spm}(A)$;

(iii) $(M(\mathfrak{M}), b(\mathfrak{M}))$ é não singular, para todo $\mathfrak{M} \in \operatorname{Spm}(A)$.

Dem.: Basta observarmos que dada uma base $\left\{x_{1}, \ldots, x_{n}\right\}$ de $M$, temos que $\left\{\frac{x_{1}}{1}, \ldots, \frac{x_{n}}{1}\right\}$ é uma base de $M_{\mathfrak{M}}$ sobre $A_{\mathfrak{M}}$ e que $\left\{x_{1}+M(\mathfrak{M}), \ldots, x_{n}+M(\mathfrak{M})\right\}$ é uma base de $M(\mathfrak{M})$ sobre $A / \mathfrak{M}$. Agora, a demostração da proposição segue de (1.3) e da definição de não singularidade. 
Outra forma de redução que usaremos no decorrer do trabalho é quando $A=F_{1} \times \ldots \times F_{r}$ é um produto finito de corpos e, $(M, b)$ um módulo bilinear sobre $A$. Neste caso, para cada $i=1, \ldots, r$ a $i$-ésima projeção canônica $\pi_{i}: A \longrightarrow F_{i}$ induz uma redução $\pi_{i}^{*}(M, b)=\left(M_{i}, b_{i}\right)$, onde $b_{i}=\pi_{i} \circ b$. De (1.10) temos que $M \cong M_{1} \times \ldots \times M_{r} \quad$ e, $\quad \pi=\left(\pi_{1}, \ldots, \pi_{r}\right)$ induz a redução

$$
\pi^{*}(M, b)=\left(M_{1}, b_{1}\right) \times \ldots \times\left(M_{r}, b_{r}\right)
$$

onde $b=\pi^{*}(b)=\left(\pi_{1} \circ b, \ldots, \pi_{r} \circ b\right)=\left(b_{1}, \ldots, b_{r}\right)$. Com estas notações temos:

Proposição 2.6 Se $A=F_{1} \times \ldots \times F_{r} \quad e \quad(M, b)$ é um módulo bilinear sobre $A$ com $M=M_{1} \times \ldots \times M_{r}$, então $b=\left(b_{1}, \ldots, b_{r}\right)$ e $(M, b)$ é não singular se, e somente se $\left(M_{i}, b_{i}\right)$ é não singular, para cada $i=1, \ldots, n$.

Dem.: Basta observar que a adjunta de $b, d_{b}=\left(d_{b_{1}}, \ldots, d_{b_{r}}\right)$ e, consequentemente, $d_{b}$ é um isomorfismo se, e somente se cada $d_{b_{i}}$ o é, para $i=1, \ldots, r$.

\subsection{Subespaços}

Seja $(M, b)$ um módulo bilinear sobre um anel $A$. Para cada subconjunto $U$ de $M$, o conjunto

$$
U^{\perp}=\{x \in M ; b(x, y)=0, \forall y \in U\},
$$

é um submódulo de $M$, chamado o complemento ortogonal de $U$ em relação à $b$. Dizemos que dois subconjuntos $U, V$ de $M$ são ortogonais se $U \subseteq V^{\perp}$ ou, equivalentemente, $V \subseteq U^{\perp}$. Com esta terminologia temos o seguinte lema de imediata verificação.

Lema 2.7 Sejam $(M, b)$ um módulo bilinear sobre $A$ e $U, V$ subconjuntos de $M$.

(i) Se $V \subseteq U$, então $U^{\perp} \subseteq V^{\perp}$; 
(ii) $U \subseteq U^{\perp \perp}$;

(iii) $U^{\perp}=U^{\perp \perp \perp}$.

Dem.: Imediata.

Um submódulo $U$ de $M$ é dito ser um subespaço de $(M, b)$ se $U$ é um somando direto de $M$. Dizemos que $x$ é um elemento primitivo de $M$ se $A x$ é um subespaço de $M$.

Se $U$ e $V$ são submódulos de $M$ tais que $M=U \oplus V$ e $U \subseteq V^{\perp}$, dizemos que $M$ é a soma ortogonal de $U$ e $V$ e denotamos por $M=U \perp V$. Denotaremos por $\left(U,\left.b\right|_{U}\right)$ a restrição da forma bilinear $b$ ao subespaço $U$ de $M$. É imediato que se $M=U \perp V$, então $(M, b) \simeq\left(U,\left.b\right|_{U}\right) \perp\left(V,\left.b\right|_{V}\right)$.

Um subconjunto $U \subseteq M$ é dito ser totalmente isotrópico se $U \subseteq U^{\perp}$. Dizemos que um elemento $x \in M$ é isotrópico se $A x$ é um subespaço totalmente isotrópico de $M$, ou seja, $x$ é um elemento primitivo de $M$ tal que $b(x, x)=0$. Um elemento $y \in M$ é dito ser anisotrópico se $A y$ é um subespaço de $M \operatorname{com} b(y, y) \in A^{\star}$. Dizemos também que o espaço bilinear $(M, b)$ é um espaço bilinear isotrópico se $M$ contém um elemento isotrópico. Se todos os elementos de $M$ são anisotrópicos dizemos que $(M, b)$ é um espaço bilinear anisotrópico.

Proposição 2.8 Seja $(M, b)$ um módulo bilinear sobre A.

(i) Se $M$ é não singular e $U \subseteq M$ é um subespaço, então $U^{\perp}$ é um subespaço de $M$ e $U=U^{\perp \perp}$.

(ii) Seja $U \subseteq M$ um submódulo tal que $\left(U,\left.b\right|_{U}\right)$ é não singular. Então $U$ é um subespaço de $M$ e $M=U \perp U^{\perp}$.

Dem.: $\operatorname{Se}(M, b)$ é não singular e $U$ é um subespaço de $M$, então existe um submódulo $V$ de $M$ tal que $M=U \oplus V$. Assim, $M^{*}=(U \oplus V)^{*} \cong U^{*} \oplus V^{*}$ e, temos a seqüência 
exata $M^{*} \longrightarrow U^{*} \longrightarrow 0$. Como $(M, b)$ é um espaço bilinear não singular, temos que a adjunta de $b, d: M \rightarrow M^{*}$ é um isomorfismo. Compondo este isomorfismo com a seqüência acima, obtemos a seqüência exata $M \longrightarrow U^{\star} \longrightarrow 0$, onde o núcleo da composta $d: M \rightarrow U^{*}$ é exatamente $U^{\perp}$. Agora, $U^{\star}$ é projetivo, pois é somando direto do $A$-módulo livre $M^{\star}$. Portanto a seqüência exata curta

$$
0 \longrightarrow U^{\perp} \longrightarrow M \longrightarrow U^{\star} \longrightarrow 0
$$

cinde, ou seja, $M \cong U^{\perp} \oplus U^{\star}$. O que mostra que $U^{\perp}$ é um subespaço de $M$.

Mostremos agora que $U^{\perp}=U^{\perp \perp}$. Para simplificar a notação, denotaremos também por $b$ as restrições da forma bilinear $b$ a subespaços de $M$. O isomorfismo $d: \frac{M}{U^{\perp}} \rightarrow U^{\star}$ induz uma forma bilinear não singular $b: U \times \frac{M}{U^{\perp}} \longrightarrow A$, para todo subespaço $U$ de $M$, onde $b(x, \bar{y})=d(\bar{y})(x)$ para todo $x \in U$ e $\bar{y} \in\left(M / U^{\perp}\right)$. Em particular, para o subespaço $U^{\perp}$ temos uma forma bilinear não singular $b: U^{\perp} \times \frac{M}{U^{\perp \perp}} \rightarrow A$. Por outro lado, temos o isomorfismo $M \cong\left(U^{\perp}\right)^{\star} \oplus U$ que decorre dos isomorfismos

$$
M^{\star} \cong\left(U^{\perp}\right)^{\star} \oplus U^{\star \star}, \quad d: M \rightarrow M^{\star} \quad \text { e } \quad U^{\star \star} \cong U .
$$

Assim obtemos o isomorfismo $d: \frac{M}{U} \longrightarrow\left(U^{\perp}\right)^{\star}$ que induz uma forma bilinear não singular $b: U^{\perp} \times \frac{M}{U} \longrightarrow A$. Destas duas bilineares e do fato que $U \subseteq U^{\perp \perp}$, segue que $U=U^{\perp \perp}$. Pois se $U \varsubsetneqq U^{\perp \perp}$, então existe $y \in U^{\perp \perp}-U$ e, das duas formas bilineares obtidas, temos $\frac{M}{U} \simeq\left(U^{\perp}\right)^{*} \simeq \frac{M}{U^{\perp \perp}}$. Como $y \in U^{\perp \perp}$, então para todo $x \in U^{\perp}$, $d(y)(x)=b(x, y)=0$. Por outro lado, como $y \notin U$, temos que $d(y) \neq 0$. Assim existe $z \in U^{\perp}$ tal que $d(y)(z) \neq 0$, ou seja, $b(z, y) \neq 0$, o que é uma contradição. Portanto $U=U^{\perp \perp}$, o que mostra (i).

Seja agora, $\left(U,\left.b\right|_{U}\right)$ um subespaço não singular de $(M, b)$. Então $d: U \rightarrow U^{\star}$ é um isomorfismo. Mas, cada elemento $x \in M$ define um elemento $x^{\star}$ em $U^{\star}$ por $x^{\star}(y)=b(x, y)=d(x)(y)$, para todo $y \in U$. Como $d: U \rightarrow U^{\star}$ é sobrejetora, temos que existe $z \in U$ tal que $d(z)=d(x)$, ou seja, $b(z, y)=b(x, y)$, para todo $y \in U$. Logo $b(x, y)-b(z, y)=0$, o que implica que $b(x-z, y)=0$, para todo $u \in U$. Assim 
$x-z \in U^{\perp}$ e, como $x=z+(x-z)$, temos que $M=U+U^{\perp}$. Do fato de $d: U \rightarrow U^{\star}$ ser injetora segue que $\{0\}=\operatorname{Ker}\left(\left.d\right|_{U}\right)=\operatorname{Ker}(d) \cap U=U^{\perp} \cap U$, o que mostra que $M=U \oplus U^{\perp}$ e, juntamente com o fato que $U \subseteq U^{\perp \perp}$ temos o item (ii).

Corolário 2.9 Sejam $M$ um espaço bilinear sobre $A$ e $U$ um subespaço totalmente isotrópico maximal de $M$, isto é, $U=U^{\perp}$. Então existe um subespaço $V$ de $M$ tal que $U \cong V^{\star}$ e $M=U \oplus V$.

Dem.: Da demonstração do item (i) da proposição anterior, temos que $M \cong U^{\perp} \oplus U^{\star}$. Tomando $V=U^{\star}$ temos $V^{\star}=U^{\star \star} \cong U$ e, como $U=U^{\perp}$, obtemos $M \cong U \oplus V$, como queríamos.

Corolário 2.10 Sejam $(M, b)$ um módulo bilinear sobre $A$ e $\mathfrak{I}$ um ideal contido no radical de Jacobson $\mathrm{J}(A)$ de $A$. Se $(M(\mathfrak{I}), b(\mathfrak{I}))$ admite uma decomposição ortogonal $M(\mathfrak{I})=N(\mathfrak{I}) \perp W(\mathfrak{I})$, com $N(\mathfrak{I})$ livre sobre $A / \mathfrak{I}$ tal que $(N(\mathfrak{I}), b(\mathfrak{I}))$ é não singular, então existe uma decomposição ortogonal $M=N \perp W$ de $(M, b)$ com $N$ livre sobre $A$ e $(N, b)$ não singular tal que $N(\mathfrak{I})=\frac{N}{\mathfrak{I} N}$ e $W(\mathfrak{I})=\frac{W}{\mathfrak{I} W}$.

Dem.: Desde que $(N(\mathfrak{I}), b(\mathfrak{I}))$ é um subespaço não singular de $(M(\mathfrak{I}), b(\mathfrak{I})) \operatorname{com} N(\mathfrak{I})$ livre sobre $A / \mathfrak{I}$, temos que existe uma base $\left\{\overline{x_{1}}, \ldots, \overline{x_{n}}\right\}$ de $N(\mathfrak{I})$ sobre $A / \mathfrak{I}$ tal que $\operatorname{det}\left(b(\mathfrak{I})\left(\overline{x_{i}}, \overline{x_{j}}\right)\right) \in(A / \mathfrak{I})^{*}$. Da proposição (1.11) temos que $N=A x_{1}+\ldots+A x_{n} \dot{\mathrm{e}}$ um $A$-módulo livre com base $\left\{x_{i}, \ldots, x_{n}\right\}$ e $N(\mathfrak{I})=\frac{N}{\mathfrak{I} N}$. A matriz da forma bilinear $\left.b\right|_{N},\left(b\left(x_{i}, x_{j}\right)\right)$, é tal que $\overline{\operatorname{det}\left(b\left(x_{i}, x_{j}\right)\right)}=\operatorname{det}\left(\overline{b\left(x_{i}, x_{j}\right)}\right)=\operatorname{det}\left(b(\mathfrak{I})\left(\overline{x_{i}}, \overline{x_{j}}\right)\right) \in(A / \mathfrak{I})^{*}$. Agora segue de (1.2) e da definição (2.1) que $(N, b)$ é um subespaço não singular de $M$, e o resultado segue de $\mathbf{( 2 . 8 )}$.

Finalizaremos esta seção mostrando que todo espaço bilinear sobre um anel semilocal admite uma decomposição como soma ortogonal de subespaços de dimensão $\leq 2$. 
Para tanto, iniciaremos com algumas definições e resultados auxiliares.

No que segue, usaremos as notações $\bar{A}$ para indicarmos o anel $A / \mathcal{g}(A)$ e $(\bar{M}, \bar{b})$ para indicarmos a redução módulo $\mathcal{\partial}(A)$ do espaço bilinear $(M, b) \in \operatorname{Bil}(A)$.

Dado $(M, b) \in \operatorname{Bil}(A)$, considere o subconjunto $\{b(x, x) ; x \in M\}$ de $A$. Se o ideal gerado por este subconjunto é todo o anel $A$, diremos que o espaço bilinear $(M, b)$ é próprio. Caso contrário, dizemos que $(M, b)$ é um espaço bilinear impróprio.

Segue imediatamente desta definição e de (1.2) que

Lema 2.11 Sejam $A$ um anel semilocal e $(M, b) \in \mathcal{B} i l(A)$. Então $(M, b)$ é um espaço bilinear próprio se, e somente se $(\bar{M}, \bar{b})$ é um espaço bilinear próprio sobre $\bar{A}$.

Dem.: Imediata.

Agora, se $A=F_{1} \times \ldots \times F_{r}$ é um produto finito de corpos e $(M, b) \in \mathcal{B} i l(A)$, da proposição (2.6), temos que $(M, b)=\left(M_{1}, b_{1}\right) \times \ldots \times\left(M_{r}, b_{r}\right), \operatorname{com}\left(M_{i}, b_{i}\right) \in \operatorname{Bil}\left(F_{i}\right)$ e $b_{i}=\pi_{i} \circ b$, para cada $i=1, \ldots, r$. Neste caso temos

Lema $2.12 O$ espaço bilinear $(M, b)$ é próprio se, e somente se $\left(M_{i}, b_{i}\right)$ é próprio, para cada $i=1, \ldots, r$.

Dem.: Basta observar que se $\mathfrak{I}$ é o ideal de $A$ gerado por $\{b(x, x) ; x \in M\}$ e, para cada $j=1, \ldots, r, \quad I_{j}$ é o ideal de $F_{j}$, gerado por $\left\{b_{j}\left(x_{j}, x_{j}\right) ; x_{j} \in M_{j}\right\}$, então $\mathfrak{I}=\mathfrak{I}_{1} \times \ldots \times \mathfrak{I}_{r}$. Assim, $\mathfrak{I}=A$ se, e somente se $\mathfrak{I}_{j}=F_{j}$, para cada $j=1, \ldots, r$.

No caso em que $A$ é um anel semilocal, temos o seguinte teorema de decomposição para espaços bilineares

Teorema 2.13 Seja $(M, b)$ um espaço bilinear sobre um anel semilocal $A$.

(i) Se $(M, b)$ é próprio, então $(M, b)$ é uma soma ortogonal de subespaços de dimensão 1 , isto é, $M$ admite uma base ortogonal em relação à forma bilinear $b$. 
(ii) $S e(M, b)$ é impróprio, então $(M, b)$ é uma soma ortogonal de subespaços de dimensão 2 da forma $\left(\begin{array}{cc}\alpha & 1 \\ 1 & \beta\end{array}\right)$, com $\alpha, \beta \in A$ tais que $1-\alpha \beta \in A^{*}$.

Dem.: Dos lemas (2.11) e (2.12), é suficiente mostrarmos o teorema para $(\bar{M}, \bar{b})$ sobre $\bar{A}=A / \partial(A)$. Podemos então assumir que $\partial(A)=\{0\}$, ou seja, que $A=$ $F_{1} \times \ldots \times F_{r}$ é um produto finito de corpos e, consequentemente $M=M_{1} \times \ldots \times M_{r}$ com $b=\left(b_{1}, \ldots, b_{r}\right)$. Também, usando indução sobre $r$, é suficiente mostrarmos o caso em que $r=2$.

Seja $(M, b)=\left(M_{1}, b_{1}\right) \times\left(M_{2}, b_{2}\right) \in \operatorname{Bil}(A)$, onde $A=F_{1} \times F_{2}$, com $F_{1}, F_{2}$ corpos. Faremos agora a demonstração por indução sobre $\operatorname{dim}(M)$. Se $\operatorname{dim}(M)=1$, nada há a demonstrar. Se $\operatorname{dim}(M)>1$ e $(M, b)$ é próprio então de (2.12) temos que $\left(M_{i}, b_{i}\right)$ é um espaço bilinear próprio sobre o corpo $F_{i}$, para cada $i=1,2$. Como o ideal gerado por $\left\{b_{i}\left(x_{i}, x_{i}\right) ; x_{i} \in M_{i}\right\}$ é $F_{i}$, para cada $i=1,2$, temos que existem $x_{1} \in M_{1}, x_{2} \in M_{2}$ tais que $b_{1}\left(x_{1}, x_{1}\right) \neq 0$ e $b_{2}\left(x_{2}, x_{2}\right) \neq 0$. Assim, $x=\left(x_{1}, x_{2}\right) \in M$ é tal que $b(x, x)=\left(b_{1}\left(x_{1}, x_{1}\right), b_{2}\left(x_{2}, x_{2}\right)\right) \in A^{*}=F_{1}^{*} \times F_{2}^{*} \mathrm{e}$, consequentemente, $(A x, b)$ é um subespaço não singular de dimensão 1 de $(M, b)$. De (2.8) temos que $(M, b)=(A x, b) \perp(W, b)$, com $W=(A x)^{\perp}$. Se $(W, b)$ é próprio, então por hipótese de indução $(W, b)$ é uma soma ortogonal de subespaços de dimensão 1 e, portanto, $(M, b)$ também o é.

$\mathrm{Se}(W, b)$ é impróprio, desde que $(W, b)=\left(W_{1}, b_{1}\right) \times\left(W_{2}, b_{2}\right)$, temos de (2.12) que $\left(W_{1}, b_{1}\right)$ é impróprio ou $\left(W_{2}, b_{2}\right)$ é impróprio. Temos dois casos a considerar:

Caso 1 - Se $\left(W_{i}, b_{i}\right)$ é impróprio para $i=1,2$. Neste caso, desde que $(W, b)$ é não singular, existem $y=\left(y_{1}, y_{2}\right), \quad z=\left(z_{1}, z_{2}\right) \in W$ tais que $b_{i}\left(y_{i}, z_{i}\right) \neq 0$ em $F_{i}$, $i=1,2$. Consequentemente, $b(y, z) \in A^{*}$. Tomando $w_{1}=x+y$ e $w_{2}=x+\lambda z$, com $\lambda=-\frac{b(x, x)}{b(y, z)} \in A$, temos que $\left(A w_{1}+A w_{2}, b\right)$ é um subespaço não singular de $(M, b)$, pois $\left(b\left(w_{;}, w_{j}\right)\right)=\left(\begin{array}{ll}b\left(w_{1}, w_{1}\right) & b\left(w_{1}, w_{2}\right) \\ b\left(w_{2}, w_{1}\right) & b\left(w_{2}, w_{2}\right)\end{array}\right)=\left(\begin{array}{cc}b(x, x) & 0 \\ 0 & b(x, x)\end{array}\right)$. Mais 
ainda, $\left\{w_{1}, w_{2}\right\}$ é uma base ortogonal deste subespaço. Assim, de (2.8)

$$
(M, b)=\left(A w_{1}, b\right) \perp\left(A w_{2}, b\right) \perp(N, b)
$$

$\operatorname{com}\left(A w_{2}, b\right) \perp(N, b)$ próprio que, por hipótese de indução, admite uma base ortogonal. Juntando esta base com $w_{1}$, formamos uma base ortogonal de $(M, b)$.

Caso 2 - Se um dos espaços $\left(W_{i}, b_{i}\right)$ é próprio, renomeando se necessário, podemos assumir que $\left(W_{1}, b_{1}\right)$ é impróprio e $\left(W_{2}, b_{2}\right)$ é próprio. Então, neste caso, existem $y_{1}, z_{1} \in W_{1}, y_{1} \neq z_{1}$, e $y_{2} \in W_{2}$ tais que $b_{1}\left(y_{1}, z_{1}\right) \neq 0$ em $F_{1}$ e $b_{2}\left(y_{2}, y_{2}\right) \neq 0$ em $F_{2}$. Agora, os elementos $w_{1}=\left(x_{1}+y_{1}, x_{2}\right)$ e $w_{2}=\left(x_{1}+\lambda z_{1}, y_{2}\right)$ de $M \operatorname{com} \lambda=-\frac{b\left(x_{1}, x_{1}\right)}{b\left(y_{1}, z_{1}\right)} \in F_{1}^{*}$, são tais que

$$
\left(b\left(w_{i}, w_{j}\right)\right)=\left(\begin{array}{cc}
\left(b_{1}\left(x_{1}, x_{1}\right), b_{2}\left(x_{2}, x_{2}\right)\right) & (0,0) \\
(0,0) & \left(b_{1}\left(x_{1}, x_{1}\right), b_{2}\left(y_{2}, y_{2}\right)\right)
\end{array}\right)
$$

ou seja $\left\{w_{1}, w_{2}\right\}$ é uma base ortogonal de um subespaço não singular de $(M, b)$ e, como no Caso 1 , temos que $(M, b)$ admite uma base ortogonal. Com isso, completamos a demostração do item (i) do teorema.

Consideremos agora que $\operatorname{dim}(M)>1$ e que $(M, b)=\left(M_{1}, b_{1}\right) \times\left(M_{2}, b_{2}\right)$ é um espaço bilinear impróprio sobre $A=F_{1} \times F_{2}$. De (2.12) temos que $\left(M_{1}, b_{1}\right)$ é impróprio ou $\left(M_{2}, b_{2}\right)$ é impróprio. Novamente temos dois casos a considerar:

Caso 1 - Se $\left(M_{i}, b_{i}\right)$ é impróprio para $i=1,2$. Neste caso, como no Caso 1 acima, existem $y, z \in M, y \neq z$, tais que $b(y, z) \in A^{*}$. O conjunto $\{y, z\}$ forma uma base de um subespaço não singular de $(M, b)$, pois a matriz da forma bilinear $b$ em relação à estes elementos é

$$
\left(\begin{array}{ll}
b(y, y) & b(y, z) \\
b(z, y) & b(z, z)
\end{array}\right)=\left(\begin{array}{cc}
0 & b(y, z) \\
b(y, z) & 0
\end{array}\right)
$$

que tem determinante inversível em $A$. Logo, mostramos que $(M, b)$ admite um subespaço não singular de dimensão 2. Trocando $z$ por $\lambda z, \operatorname{com} \lambda=\frac{1}{b(y, z)}$, temos que 
$(M, b)$ admite um subespaço não singular da forma $\left(\begin{array}{cc}\alpha & 1 \\ 1 & \beta\end{array}\right), \operatorname{com} 1-\alpha \beta=1 \in A^{*}$.

Caso 2 - Se um dos espaços $\left(M_{i}, b_{i}\right)$ é próprio. Sem perda de generalidade, podemos supor que $\left(M_{1}, b_{1}\right)$ é impróprio e $\left(M_{2}, b_{2}\right)$ é próprio. Como no Caso 2 anterior, podemos obter $\left\{y_{1}, z_{1}\right\} \subseteq W_{1}, \quad\left\{y_{2}, z_{2}\right\} \subseteq W_{2}$ tais que $b_{1}\left(y_{1}, z_{1}\right) \neq 0, b_{1}\left(y_{1}, y_{1}\right)=$ $b_{1}\left(z_{1}, z_{1}\right)=0, \quad b_{2}\left(y_{2}, z_{2}\right)=0, \quad b_{2}\left(y_{2}, y_{2}\right) \neq 0 \quad$ e $\quad b_{2}\left(z_{2}, z_{2}\right) \neq 0$. Tomando $w_{1}=\left(y_{1}, y_{2}+z_{2}\right)$ e $w_{2}=\left(\lambda_{1} z_{1}, \lambda_{2} z_{2}\right)$ em $M$, onde $\lambda_{1}=\frac{1}{b_{1}\left(y_{1}, z_{1}\right)} \in F_{1}^{*}$ e $\lambda_{2}=$ $\frac{1}{b_{2}\left(z_{2}, z_{2}\right)} \in F_{2}^{*}$, temos que $\left\{w_{1}, w_{2}\right\}$ é uma base de um subespaço de dimensão 2 de $(M, b)$ e, a matriz da forma bilinear restrita à este subespaço é $\left(b\left(w_{i}, w_{j}\right)\right)=$ $\left(\begin{array}{ll}\alpha & 1 \\ 1 & \beta\end{array}\right)$ onde $\alpha=b\left(w_{1}, w_{1}\right)=\left(0, b_{2}\left(y_{2}, y_{2}\right)+b_{2}\left(z_{2}, z_{2}\right)\right) \in A$ e $\beta=b\left(w_{2}, w_{2}\right)=$ $\left(0, \lambda_{2}^{2} b_{2}\left(z_{2}, z_{2}\right)\right) \in A$ são tais que $1-\alpha \beta=(1,1)-\left(0, \frac{b_{2}\left(y_{2}, y_{2}\right)}{b_{2}\left(z_{2}, z_{2}\right)}+1\right)=$ $\left(1,-\frac{b_{2}\left(y_{2}, y_{2}\right)}{b_{2}\left(z_{2}, z_{2}\right)}\right) \in A^{*}$. Agora, o item (ii) do teorema segue de (2.8) e da hipótese de indução, pois todo subespaço não singular de um espaço bilinear impróprio é também impróprio.

Corolário 2.14 Todo espaço bilinear próprio sobre um anel semilocal $A$ é da forma $\left\langle\alpha_{1}, \ldots, \alpha_{n}\right\rangle, \operatorname{com} \alpha_{i} \in A^{\star}, 1 \leq i \leq n$.

Dem.: Imediata.

\subsection{Espaços Metabólicos e Hiperbólicos}

Encerramos este capítulo com a definição e a caracterização dos espaços metabólicos e hiperbólicos, os quais são essenciais para a definição dos anéis de Witt que 
apresentaremos no próximo capítulo.

Seja $(U, b)$ um módulo bilinear sobre $A$. Definimos em $U \oplus U^{*}$ uma forma bilinear simétrica $b_{U}$ por:

$$
b_{U}\left(u+u^{\star}, v+v^{\star}\right)=b(u, v)+u^{\star}(v)+v^{\star}(u)
$$

para todo $u, v \in U$ e $u^{\star}, v^{\star} \in U^{\star}$. Ao módulo bilinear $\left(U \oplus U^{\star}, b_{U}\right)$ damos o nome de espaço metabólico e denotamos por $\mathbb{M}(U, b)$, ou simplesmente por $\mathbb{M}(U)$, ou ainda $\mathbb{M}(b)$. Provamos a seguir que este módulo é de fato não singular.

Proposição 2.15 Para todo módulo bilinear $(U, b)$ sobre $A, \quad \mathrm{M}(U)$ é um espaço bilinear.

Dem.: Consideremos $\left\{e_{1}, \ldots, e_{n}, e_{1}^{\star}, \ldots, e_{n}^{\star}\right\}$ uma base de $\mathbb{M}(U)=U \oplus U^{\star}$, onde $\left\{e_{1}, \ldots, e_{n}\right\}$ é uma base de $U$ e $\left\{e_{1}^{\star}, \ldots, e_{n}^{\star}\right\}$ é a base dual de $U^{\star}$. A matriz de $b_{U}$ com relação a esta base é a matriz em blocos

$$
\left(b_{U}\left(e_{i}, e_{j}^{\star}\right)\right)=\left(\begin{array}{cc}
b & I \\
I & 0
\end{array}\right)
$$

onde o bloco $b$ é a matriz associada ao módulo bilinear $(U, b), I$ é a matriz identidade $n \times n$ e 0 é a matriz nula $n \times n$. O determinante desta matriz é $-\operatorname{det}(I) \operatorname{det}(I)$, isto é, a matriz associada a forma bilinear simétrica $b_{U}$ é inversivel e, portanto $\mathbb{M}(U)$ é um espaço bilinear não singular, como queríamos.

Em $\mathbb{M}(U)$ o subespaço $U^{\star}$ é sempre totalmente isotrópico, o mesmo pode não ocorrer com $U$. Se $b=0$, então $U$ também é um subespaço totalmente isotrópico de $\mathbb{M}(U)$. Neste caso, dizemos que $\mathbb{M}(U, 0)$ é um espaço bilinear hiperbólico que denotaremos também por $\mathbb{H}(U)$.

Se $U=A x$, então $\mathbb{H}(U)=\left(\begin{array}{ll}0 & 1 \\ 1 & 0\end{array}\right)$, será chamado de plano hiperbólico e denotado simplesmente por $\mathbb{H}$. Todo espaço bilinear hiperbólico é uma soma ortogonal 
de planos hiperbólicos. De fato, consideremos $(U, 0)$ tal que $\operatorname{dim}(U)=n$. Sejam $\left\{e_{1}, \cdots, e_{n}\right\}$ e $\left\{e_{1}^{*}, \cdots, e_{n}^{*}\right\}$ bases de $U$ e $U^{*}$, respectivamente. Podemos considerar $\left\{e_{1}, e_{1}^{\star}, e_{2}, e_{2}^{\star}, \cdots, e_{n}, e_{n}^{\star}\right\}$ como base de $U \oplus U^{\star}$ e em relação a esta base

$$
\mathbf{H}(U) \simeq\left(\begin{array}{ll}
0 & 1 \\
1 & 0
\end{array}\right) \perp \ldots \perp\left(\begin{array}{ll}
0 & 1 \\
1 & 0
\end{array}\right)
$$

com $n$ parcelas. Portanto, $\mathbb{H}(U) \simeq n \mathbb{H}$, onde $n=\operatorname{dim}(U)$.

Consideremos agora, $(U, b)=(A x,\langle\alpha\rangle)$, então

$$
\mathbf{M}(\langle\alpha\rangle)=\left(\begin{array}{ll}
\alpha & 1 \\
1 & 0
\end{array}\right)
$$

Mais geralmente,

$$
\mathbf{M}\left(\left\langle\alpha_{1}, \ldots, \alpha_{n}\right\rangle\right) \simeq\left(\begin{array}{cc}
\alpha_{1} & 1 \\
1 & 0
\end{array}\right) \perp \ldots \perp\left(\begin{array}{cc}
\alpha_{n} & 1 \\
1 & 0
\end{array}\right)
$$

Proposição 2.16 Se 2 é uma unidade em A, então todo espaço metabólico é hiperbólico. Além disso, $\mathbb{H} \simeq\langle 1,-1\rangle$.

Dem.: Das decomposições ortogonais de $\mathbb{H}(U)$ e de $\mathbf{M}(U)$ listadas acima vemos que é suficiente mostrarmos que, para todo $\alpha \in A$, temos

$$
\left(\begin{array}{ll}
\alpha & 1 \\
1 & 0
\end{array}\right) \simeq\left(\begin{array}{ll}
0 & 1 \\
1 & 0
\end{array}\right)
$$

Seja $(A x \oplus A y, b)=\left(\begin{array}{ll}\alpha & 1 \\ 1 & 0\end{array}\right)$, ou seja, $b(x, x)=\alpha, b(x, y)=1$ e $b(y, y)=0$. Os elementos $x^{\prime}=x-\frac{\alpha}{2} y$ e $y^{\prime}=y \in A x \oplus A y$ são tais que $b\left(x^{\prime}, x^{\prime}\right)=0=b\left(y^{\prime}, y^{\prime}\right)$ e $b\left(x^{\prime}, y^{\prime}\right)=1$. Assim $\left(A x^{\prime} \oplus A y^{\prime}, b\right)=\left(\begin{array}{ll}0 & 1 \\ 1 & 0\end{array}\right)$ é um subespaço não singular de 
$\left(\begin{array}{ll}\alpha & 1 \\ 1 & 0\end{array}\right)$. Agora,de (2.8) e do fato que ambos são espaços bilineares de dimensão 2 , temos que

$$
\left(\begin{array}{ll}
\alpha & 1 \\
1 & 0
\end{array}\right) \simeq\left(\begin{array}{ll}
0 & 1 \\
1 & 0
\end{array}\right)
$$

Além disso, se $\mathbb{H}=\left(\begin{array}{ll}0 & 1 \\ 1 & 0\end{array}\right)$, com base $\left\{x^{\prime}, y^{\prime}\right\}$ como acima, então $A x^{\prime \prime} \oplus A y^{\prime \prime}$, onde $x^{\prime \prime}=\frac{x^{\prime}+y^{\prime}}{2}$ e $y^{\prime \prime}=\frac{x^{\prime}-y^{\prime}}{2}$, é um subespaço não singular de $\mathbb{H}$ da forma $\langle 1,-1\rangle$ e, como acima, obtemos que $\mathbb{H} \simeq\langle 1,-1\rangle$.

Teorema 2.17 Seja $(M, b)$ um espaço bilinear sobre $A$. Se $U \subseteq M$ é um subespaço totalmente isotrópico de $M$, então existe um subespaço $V \subseteq M$ tal que $M=U^{\perp} \oplus V$ $e(U \oplus V, b)$ é metabólico.

Dem.: De (2.8) temos que $U^{\perp}$ é subespaço de $M$ e então existe $V \subseteq M$ tal que $M=U^{\perp} \oplus V$. Mais ainda, da demonstração de (2.8), temos que $b: U \times M / U^{\perp} \longrightarrow A$ é não singular, isto é, $b: U \times V \longrightarrow A$ é não singular. Obtemos assim o isomorfismo $d: U \longrightarrow V^{*}$. Temos que $U \cap V=\{0\}$, pois $U \subseteq U^{\perp}$ e $U^{\perp} \cap V=\{0\}$. Assim $U \oplus V$ é um subespaço de $M$. Mostremos que $b:(U \oplus V) \times(U \oplus V) \longrightarrow A$ é não singular. Como $U \subseteq U^{\perp}$, a matriz de $b$ em relação a decomposição $(U \oplus V) \times(U \oplus V)=$ $(U \times U) \oplus(U \times V) \oplus(V \times U) \oplus(V \times V)$, é a matriz em blocos

$$
B=\left(\begin{array}{cc}
0 & B_{12} \\
B_{12} & B_{22}
\end{array}\right)
$$

onde $B_{12}$ é a matriz de $\left.b\right|_{U \times V}$ e $B_{22}$ é a matriz de $\left.b\right|_{V \times V}$. Como $b: U \times V \longrightarrow A$ é não singular então $B_{12}$ é inversível. Assim

$$
B^{-1}=\left(\begin{array}{cc}
-B_{12}^{-1} B_{22} B_{12}^{-1} & B_{12}^{-1} \\
B_{12}^{-1} & 0
\end{array}\right)
$$


é a inversa de $B$, donde concluimos que $B$ é inversível, ou seja, $(U \oplus V, b)$ é não singular e de (2.8) temos que $M=(U \oplus V) \perp(U \oplus V)^{\perp}$.

Seja $f: U \oplus V \longrightarrow \mathbb{M}(V)=V \oplus V^{*}$ definida por $f(u+v)=v+d(u)$, para todo $u \in U$ e $v \in V$. Temos que $f$ é um isomorfismo, pois é a soma da identidade com o isomorfismo $d$. Mostremos que de fato $f$ é uma isometria. Para todo $u+v, u^{\prime}+v^{\prime}$ em $U \oplus V$, temos

$$
\begin{aligned}
& b_{V}\left(f(u+v), f\left(u^{\prime}+v^{\prime}\right)\right)=b_{V}\left(v+d(u), v^{\prime}+d\left(u^{\prime}\right)\right)=b\left(v, v^{\prime}\right)+d(u)\left(v^{\prime}\right)+d\left(u^{\prime}\right)(v)= \\
& =b\left(v, v^{\prime}\right)+b\left(u, v^{\prime}\right)+b\left(u^{\prime}, v\right)=b\left(u+v, v^{\prime}\right)+b\left(u^{\prime}, v+u\right)=b\left(u+v, u^{\prime}+v^{\prime}\right) . \text { Assim, } \\
& U \oplus V \simeq \mathbb{M}(V), \text { donde concluimos que }(U \oplus V, b) \text { é metabólico. }
\end{aligned}
$$

Corolário 2.18 Se $x \in(M, b)$ é um elemento primitivo e isotrópico, então existe $y \in M$ tal que $(A x \oplus A y, b)=\left(\begin{array}{cc}0 & 1 \\ 1 & b(y, y)\end{array}\right)$ é um subespaço metabólico não singular $d e(M, b)$.

Dem.: No teorema anterior, consideremos $U=A x$, temos que existe $V=A y^{\prime}$ tal que $\left(A x \oplus A y^{\prime}, b\right)$ é não singular e metabólico. A matriz de $b$ com relação a base $\left\{x, y^{\prime}\right\}$ é dada por $\left(\begin{array}{cc}0 & b\left(x, y^{\prime}\right) \\ b\left(x, y^{\prime}\right) & b\left(y^{\prime}, y^{\prime}\right)\end{array}\right)$. Como $b$ é não singular, temos que $b\left(x, y^{\prime}\right)$ é uma unidade. Tomando $y=\frac{1}{b\left(x, y^{\prime}\right)} y^{\prime}$, temos que a matriz de $b$ com relação a base $\{x, y\}$ é $\left(\begin{array}{cc}0 & 1 \\ 1 & b(y, y)\end{array}\right)$, como queríamos.

O próximo teorema caracteriza os espaços metabólicos.

Teorema 2.19 Seja $(M, b)$ um espaço bilinear sobre $A$. Então $(M, b)$ é um espaço metabólico se, e somente se $M$ contém um subespaço $U$ totalmente isotrópico maximal, isto é, $U=U^{\perp}$. 
Dem.: Seja $(M, b)$ um espaço metabólico. Então $M=V \oplus V^{*}$ para algum módulo bilinear $\left(V, b^{\prime}\right)$. Como $U=V^{*}$ é um subespaço totalmente isotrópico, temos que $V^{*} \subseteq\left(V^{*}\right)^{\perp}$. Vamos mostrar que $\left(V^{*}\right)^{\perp} \subseteq V^{*}$, ou seja, que $V^{*}$ é um subespaço totalmente isotrópico maximal de $M$. Consideremos $\left\{x_{1}, \ldots, x_{n}\right\}$ uma base de $V$ e $\left\{x_{1}^{*}, \ldots, x_{n}^{*}\right\}$ a base dual de $V^{*}$. Para $v+v^{*} \in\left(V^{*}\right)^{\perp}$, temos $b_{V}\left(v+v^{*}, v_{1}^{*}\right)=0$, para todo $v_{1}^{*} \in V^{*}$, em particular, $b_{V}\left(v+v^{*}, x_{i}^{*}\right)=0$, para todo $i=1, \ldots, n$. Mas $b_{V}\left(v+v^{*}, x_{i}^{*}\right)=b(v, 0)+v^{*}(0)+x_{i}^{*}(v)=0$, ou seja, $x_{i}^{*}(v)=0$, para todo $i=1, \ldots, n$. Escrevendo $v=u_{1} x_{1}+\ldots+u_{n} x_{n}$, temos $0=x_{i}^{*}(v)=x_{i}^{*}\left(u_{1} x_{1}+\ldots+u_{n} x_{n}\right)=u_{i}$, para todo $i=1, \ldots, n$. Logo $v=0$ e, assim $v+v^{*}=v^{*} \in V^{*}$, ou seja, $\left(V^{*}\right)^{\perp} \subseteq V^{*}$. Portanto $V^{*}=\left(V^{*}\right)^{\perp}$. A recíproca segue imediatamente do teorema (2.17).

Corolário 2.20 $\operatorname{Seja}(V, b) \in \mathcal{B} i l(A)$.

(i) $S e\left(U, b^{\prime}\right)$ é um módulo bilinear sobre $A$, então $\mathbb{M}(U) \otimes(V, b) \simeq \mathbb{M}(U \otimes V)$.

(ii) $(V, b) \perp(V,-b) \simeq \mathbb{M}(V)$, onde $-b(x, y)=(-1) b(x, y)$, para todo $x, y \in V$.

Dem.: Desde que $\mathbb{M}(U) \otimes V=\left(U \oplus U^{\star}\right) \otimes V \cong(U \otimes V) \oplus\left(U^{\star} \otimes V\right)$ e $U^{\star}$ é um subespaço totalmente isotrópico de $\mathbb{M}(U)$, temos que $U^{\star} \otimes V$ é um subespaço totalmente isotrópico de $\mathbb{M}(U) \otimes V$, ou seja, $U^{\star} \otimes V \subseteq\left(U^{\star} \otimes V\right)^{\perp}$. Para provarmos (i), usando o teorema anterior, é suficiente mostrarmos que $\left(U^{\star} \otimes V\right)^{\perp} \subseteq U^{\star} \otimes V$, ou seja, que $U^{\star} \otimes V$ é um subespaço totalmente isotrópico maximal de $\mathbb{M}(U) \otimes V$. Desde que $\mathbb{M}(U) \otimes V$ é gerado pelos elementos da forma $\left(u \oplus u^{\star}\right) \otimes v$, com $u \in U$ e $v \in V$, é suficiente considerarmos os elementos de $\left(U^{\star} \otimes V\right)^{\perp}$ da forma $\left(u_{i} \oplus u_{i}^{*}\right) \otimes v_{i}$, com $u_{i} \in U$ e $v_{i}$ em alguma base de $V$. Para tais elementos temos

$$
\left(b_{U} \otimes b\right)\left(\left(u_{i}+u_{i}^{*}\right) \otimes v_{i}, u^{*} \otimes v\right)=0
$$

para todo $u^{\star} \in U^{\star}$ e $v \in V$. Como $v_{i}$ pertence a alguma base de $V$ e $(V, b)$ é não singular, temos que existe $v \in V$ tal que $b\left(v_{i}, v\right) \in A^{\star}$. Assim $0=b_{U}\left(u_{i}+u_{i}^{*}, u^{*}\right)$. 
$b\left(v_{i}, v\right)$, para todo $u^{\star} \in U^{\star}$, o que implica que $0=b_{U}\left(u_{i}+u_{i}^{\star}, u^{\star}\right)=b\left(u_{i}, 0\right)+u_{i}^{\star}(0)+$ $u^{\star}\left(u_{i}\right)$, ou seja, $u^{\star}\left(u_{i}\right)=0$ para todo $u^{\star} \in U^{\star}$. Logo $u_{i}=0$, o que mostra (i).

Para o item (ii) basta observarmos que $U=\{(x, x) ; x \in V\}$ é um subespaço totalmente isotrópico maximal de $(V, b) \perp(V,-b)$.

Proposição 2.21 Seja $(U, b)$ um módulo bilinear. Então:

$$
\mathbb{M}(U, b) \perp \mathbb{M}(U,-b) \simeq \mathbb{H}(U) \perp \mathbb{M}(U,-b) .
$$

Dem.: Seja $\left\{x_{1}, \ldots, x_{n}, x_{1}^{*}, \ldots, x_{n}^{*}\right\}$ uma base de $U \oplus U^{*}$, onde $\left\{x_{1}, \ldots, x_{n}\right\}$ é uma base de $U$ e $\left\{x_{1}^{\star}, \ldots, x_{n}^{\star}\right\}$ é a base dual de $U^{\star}$. Nesta base temos que

$$
\mathbb{M}(U, b)=\left(\begin{array}{ll}
0 & I \\
I & b
\end{array}\right),
$$

onde $I$ é a matriz identidade $n \times n, 0$ é a matriz nula $n \times n$ e $b$ é a matriz de $(U, b)$ em relação a base dada. Note que em relação a esta mesma base

$$
\mathbb{M}(U,-b)=\left(\begin{array}{ll}
0 & -I \\
-I & -b
\end{array}\right),
$$

pois, $-b_{U}(x, y)=-b(x, y)-u^{\star}(v)-v^{\star}(u)$.

Considerando a matriz em blocos inversível $C=\left(\begin{array}{cccc}I & 0 & I & b \\ 0 & I & 0 & 0 \\ 0 & 0 & I & 0 \\ 0 & I & 0 & I\end{array}\right)$, obtemos

$$
\left(\begin{array}{llll}
I & 0 & 0 & 0 \\
0 & I & 0 & I \\
I & 0 & I & 0 \\
b & 0 & 0 & I
\end{array}\right)\left(\begin{array}{cccc}
0 & I & 0 & 0 \\
I & b & 0 & 0 \\
0 & 0 & 0 & -I \\
0 & 0 & -I & -b
\end{array}\right)\left(\begin{array}{llll}
I & 0 & I & b \\
0 & I & 0 & 0 \\
0 & 0 & I & 0 \\
0 & I & 0 & I
\end{array}\right)=\left(\begin{array}{cccc}
0 & I & 0 & 0 \\
I & 0 & 0 & 0 \\
0 & 0 & 0 & -I \\
0 & 0 & -I & -b
\end{array}\right),
$$

o que mostra que $\mathbb{M}(U, b) \perp \mathbb{M}(U,-b) \simeq \mathbb{H}(U) \perp \mathbb{M}(U, b)$, como queríamos. 


\section{Capítulo 3}

\section{O Anel de Witt}

Neste capítulo apresentaremos um estudo da estrutura do anel $\mathcal{W}(A)$, o anel de Witt dos espaços bilineares sobre um anel semilocal $A$. Mais especificamente, daremos uma descrição dos ideais primos e dos geradores de $\mathcal{W}(A)$. Apresentaremos também um estudo dos elementos de torção, dos elementos nilpotentes e dos divisores de zero de $\mathcal{W}(A)$.

\subsection{Geradores de $\mathcal{W}(A)$}

À categoria $\operatorname{Bil}(A)$ associamos seu correspondente Anel de Grothendieck, o qual é chamado, Anel de Witt-Grothendieck dos espaços bilineares sobre $A$. Tal anel será denotado por $\widehat{\mathcal{W}}(A)$. Se [b] denota a classe de isometrias do espaço bilinear $b$, então os elementos de $\widehat{\mathcal{W}}(A)$ são as diferenças formais $\left[b_{1}\right]-\left[b_{2}\right]$, de classes $\left[b_{1}\right]$ e $\left[b_{2}\right]$, onde por definição $\left[b_{1}\right]-\left[b_{2}\right]=\left[b_{1}^{\prime}\right]-\left[b_{2}^{\prime}\right]$ se, e somente se existe $b \in \mathcal{B i l}(A)$ tal que $b_{1} \perp b_{2}^{\prime} \perp b \simeq b_{2} \perp b_{1}^{\prime} \perp b$.

As operações que fornecem uma estrutura de anel comutativo em $\widehat{\mathcal{W}}(A)$ são as operações induzidas pelas operações soma ortogonal e produto tensorial de $\operatorname{Bil}(A)$.

Seja $\widehat{\mathbb{M}}(A)=\left\{[b]-\left[b^{\prime}\right] \in \widehat{\mathcal{W}}(A) ; b\right.$ e $b^{\prime}$ são metábolicos $\}$. De (2.20), decorre que $\widehat{\mathbb{M}}(A)$ é um ideal de $\widehat{\mathcal{W}}(A)$. Assim definimos o anel de Witt dos espaços bilineares 
sobre $A$ como sendo o anel quociente $\mathcal{W}(A)=\frac{\widehat{W}(A)}{\widehat{\mathrm{M}}(A)}$.

Com a mesma notação de $\widehat{\mathcal{W}}(A)$, seja $\left[b_{1}\right]-\left[b_{2}\right]$ um elemento genérico de $\mathcal{W}(A)$. Então

$$
\left[b_{1}\right]-\left[b_{2}\right]=\left[b_{1}\right]-\left[b_{2}\right]+\left[\left(-b_{2}\right)\right]-\left[\left(-b_{2}\right)\right]=\left[b_{1} \perp\left(-b_{2}\right)\right]-\left[b_{2} \perp\left(-b_{2}\right)\right]
$$

De (2.20) temos que $b_{2} \perp\left(-b_{2}\right)$ é metabólico; logo $\left[b_{2} \perp\left(-b_{2}\right)\right]=[0]$ em $\mathcal{W}(A)$. Assim, $\left[b_{1}\right]-\left[b_{2}\right]=\left[b_{1} \perp\left(-b_{2}\right)\right]$, ou seja, todo elemento de $\mathcal{W}(A)$ pode ser escrito na da forma $[b], \operatorname{com} b$ em $\operatorname{Bil}(A)$.

Da definição de $\mathcal{W}(A)$ segue que $\left[b_{1}\right]=\left[b_{2}\right]$ se, e somente se existem $U, V$ módulos bilineares sobre $A$ tais que $b_{1} \perp \mathrm{M}(U) \simeq b_{2} \perp \mathrm{M}(V)$.

Vemos facilmente que $\mathcal{W}(A)$, com as operações induzidas por $\perp \mathrm{e} \otimes$, é de fato um anel comutativo com elemento identidade, onde $-[b]=[(-b)]$ e $1_{\mathcal{W}(A)}=[\langle 1\rangle]$. Quando não houver perigo de confusão denotaremos simplesmente por $b$ o elemento [b] de $\mathcal{W}(A)$, dentro do contexto se tornará claro quando consideramos $b$ como um elemento de $\mathcal{W}(A)$ ou como um elemento de $\operatorname{Bil}(A)$.

Decorre de (2.21) que, para todo módulo bilinear $(U, b), \mathbf{M}(U)=\mathbf{H}(U)$ em $\mathcal{W}(A)$. Desta forma se considerarmos o subconjunto de $\widehat{\mathcal{W}}(A)$

$$
\widehat{\mathbb{H}}(A)=\{[\mathbb{H}(U)]-[\mathbb{H}(V)] ; U, V \text { são módulos bilineares }\} \text {, }
$$

temos que $\widehat{\mathrm{H}}(A)=\widehat{\mathrm{M}}(A)$ em $\widehat{\mathcal{W}}(A)$. Agora se $\operatorname{dim}(U)=m$ e $\operatorname{dim}(V)=n$ temos que $\mathbb{H}(U) \simeq m \mathbb{H}$ e $\mathbb{H}(V) \simeq n \mathbb{H}$, ou seja em $\mathcal{W}(A)$ temos

$$
[\mathbb{H}(U)]-[\mathbb{H}(V)]=[m \mathbb{H}]-[n \mathbb{H}]=[(m-n) \mathbb{H}]
$$

com $m-n \in \mathbb{Z}$. Portanto, podemos identificar $\widehat{\mathbb{H}}(A) \operatorname{com} \mathbb{Z} \mathbb{H}=\{n \mathbb{H} ; n \in \mathbb{Z}\}$ e escrever $\mathcal{W}(A)=\frac{\widehat{\mathcal{W}}(A)}{\mathbb{Z} \mathbb{H}}$.

Proposição 3.1 Dois espaços bilineares são iguais em $\widehat{\mathcal{W}}(A)$ se, e somente se são iguais em $\mathcal{W}(A)$ e tem a mesma dimensão. 
Dem.: Se $\left[b_{1}\right]=\left[b_{2}\right]$ em $\widehat{\mathcal{W}}(A)$, então existe $(V, b) \in \operatorname{Bil}(A)$ tal que $b_{1} \perp b \simeq$ $b_{2} \perp b$ e, consequentemente, $\operatorname{dim}\left(b_{1}\right)=\operatorname{dim}\left(b_{2}\right)$. Além disso temos $b_{1} \perp b \perp(-b) \simeq$ $b_{2} \perp b \perp(-b)$ o que implica, de (2.20), que $b_{1} \perp \mathbb{M}(V) \simeq b_{2} \perp \mathbb{M}(V)$, ou seja, $\left[b_{1}\right]=$ $\left[b_{2}\right]$ em $\mathcal{W}(A)$. Reciprocamente, sejam $b_{1}, b_{2} \in \mathcal{B} i l(A)$ espaços de mesma dimensão tais que $\left[b_{1}\right]=\left[b_{2}\right]$ em $\mathcal{W}(A)$. Então existem $m, n \in \mathbb{Z}$ tais que $b_{1} \perp m \mathbb{H} \simeq b_{2} \perp n \mathbb{H}$. Como $\operatorname{dim}\left(b_{1}\right)=\operatorname{dim}\left(b_{2}\right)$, igualando as dimensões temos que $m=n$. Portanto, $\left[b_{1}\right]=\left[b_{2}\right]$ em $\widehat{\mathcal{W}}(A)$.

Sejam $G=A^{\star} / A^{\star^{2}}$ o grupo das classes quadradas de $A$ e $f: G \rightarrow \mathcal{W}(A)$ a aplicação que leva cada classe $(\alpha) \in G$ no elemento $[\langle\alpha\rangle] \in \mathcal{W}(A)$. Escrevemos $f(\alpha)$ para indicar a imagem de $(\alpha)$ pela aplicação $f$.

Como um primeiro resultado sobre a geração do anel de Witt temos

Teorema 3.2 $O$ anel $\mathcal{W}(A)$ é aditivamente gerado por $f(G)$, a imagem de $f$.

Dem.: Seja $b \in \mathcal{B} i l(A)$. O espaço bilinear $b \perp\langle 1\rangle$ é próprio e, de (2.13), $b \perp\langle 1\rangle$ admite uma base ortogonal, ou seja, existem $\beta_{1}, \ldots, \beta_{n} \in A^{\star}$ tais que:

$$
b \perp\langle 1\rangle \simeq\left\langle\beta_{1}\right\rangle \perp \ldots \perp\left\langle\beta_{n}\right\rangle .
$$

Desde que $\langle 1,-1\rangle$ é metabólico, em $\mathcal{W}(A)$ temos

$$
b=b \perp\langle 1,-1\rangle=\left\langle\beta_{1}\right\rangle \perp \ldots \perp\left\langle\beta_{n}\right\rangle \perp\langle-1\rangle=f\left(\beta_{1}\right)+\ldots+f\left(\beta_{n}\right)+f(-1) .
$$

Assim, todo elemento de $\mathcal{W}(A)$ se escreve como uma soma finita de elementos de $f(G)$.

Observação $3.3 \mathrm{O}$ resultado acima mostra, em particular, que todo elemento de $\mathcal{W}(A)$ pode ser representado pela classe de um espaço bilinear próprio sobre $A$, independentemente de 2 ser ou não inversível no anel $A$. 
Seja $\mathbb{Z}[G]$ o anel de grupos de $G$. Também do teorema anterior, podemos afirmar que existe um homomorfismo de anéis sobrejetor $\varphi: \mathbb{Z}[G] \rightarrow \mathcal{W}(A)$, que é a extensão por linearidade de $f$. $O$ próximo resultado caracteriza o núcleo $\mathcal{K}$ deste homomorfismo.

Proposição 3.4 $O$ ideal $\mathcal{X}$ de $\mathbb{Z}[G]$ é aditivamente gerado por $(1)+(-1)$ e por todos os elementos da forma $\sum_{i=1}^{n}\left(\alpha_{i}\right)-\sum_{i=1}^{n}\left(\beta_{i}\right) \in \mathbb{Z}[G]$, com $n \in \mathbb{N}$, tais que

$$
\left\langle\alpha_{1}, \ldots, \alpha_{n}\right\rangle \simeq\left\langle\beta_{1}, \ldots, \beta_{n}\right\rangle
$$

Dem.: Claramente os elementos deste tipo estão em $\mathcal{K}$. Por outro lado, seja $z=\sum_{i=1}^{r}\left(\alpha_{i}\right)-\sum_{i=1}^{s}\left(\beta_{i}\right)$ um elemento de $\mathcal{X}$. Trocando $z$ por $-z$ se necessário, podemos assumir que $r \geq s$. Desde que $\varphi(z)=0$, temos que $\left\langle\alpha_{1}, \ldots, \alpha_{r}\right\rangle=\left\langle\beta_{1}, \ldots, \beta_{s}\right\rangle$ em $\mathcal{W}(A)$, ou seja, existem $U_{1}, U_{2} \in \operatorname{Bil}(A)$, tais que

$$
\left\langle\alpha_{1}, \ldots, \alpha_{r}\right\rangle \perp \mathbb{M}\left(U_{1}\right) \simeq\left\langle\beta_{1}, \ldots, \beta_{s}\right\rangle \perp \mathbb{M}\left(U_{2}\right) .
$$

Como, $\operatorname{dim}\left(\mathbb{M}\left(U_{1}\right)\right)$ e $\operatorname{dim}\left(\mathbb{M}\left(U_{2}\right)\right)$ são números pares, temos que $r-s$ é um número par, digamos $2 t, \operatorname{com} t \geq 0$.

Sejam $b_{1}=\left\langle\alpha_{1}, \ldots, \alpha_{r}\right\rangle$ e $b_{2}=\left\langle\beta_{1}, \ldots, \beta_{s}\right\rangle \perp t\langle 1,-1\rangle$. Desde que $b_{1}=b_{2}$ em $\mathcal{W}(A)$ e $\operatorname{dim}\left(b_{1}\right)=\operatorname{dim}\left(b_{2}\right)$, temos por (3.1) que eles representam o mesmo elemento em $\widehat{\mathcal{W}}(A)$. Assim, existe $b_{3} \in \mathcal{B} i l(A)$ tal que

$$
\left\langle\alpha_{1}, \ldots, \alpha_{r}\right\rangle \perp b_{3} \simeq\left\langle\beta_{1}, \ldots, \beta_{s}\right\rangle \perp t\langle 1,-1\rangle \perp b_{3} .
$$

Somando $\langle 1\rangle$ em ambos os lados, se necessário, podemos assumir que $b_{3}$ é próprio, ou seja $b_{3} \simeq\left\langle\alpha_{r+1}, \ldots, \alpha_{n}\right\rangle$. Tomando $\beta_{i}= \pm 1$ para $s<i \leq r$ e $\beta_{i}=\alpha_{i}$ para $r<i \leq n$, obtemos $\left\langle\alpha_{1}, \ldots, \alpha_{n}\right\rangle \simeq\left\langle\beta_{1}, \ldots, \beta_{n}\right\rangle$ e $z=t(\langle 1)+(-1))+\sum_{i=1}^{n}\left(\alpha_{i}\right)-\sum_{i=1}^{n}\left(\beta_{i}\right)$, como queríamos.

Teorema 3.5 $O$ anel $\mathcal{W}(A)$ é aditivamente gerado por $\left\{\langle\alpha\rangle ; \alpha \in A^{\star}\right\}$ com as seguintes relações: 
(i) $\left\langle\alpha \beta^{2}\right\rangle=\langle\alpha\rangle$, para todo $\beta \in A^{*}$.

(ii) $\left\langle\alpha_{1}\right\rangle+\cdots+\left\langle\alpha_{n}\right\rangle=\left\langle\beta_{1}\right\rangle+\cdots+\left\langle\beta_{n}\right\rangle \Longleftrightarrow\left\langle\alpha, \ldots, \alpha_{n}\right\rangle \simeq\left\langle\beta_{1}, \ldots, \beta_{n}\right\rangle$.

(iii) $\langle\alpha\rangle+\langle-\alpha\rangle=0$.

(iv) $\langle\alpha\rangle+\langle\beta\rangle=\langle\alpha+\beta\rangle+\langle\alpha \beta(\alpha+\beta)\rangle$, se $\alpha+\beta \in A^{\star}$.

(v) $\langle\alpha\rangle\langle\beta\rangle=\langle\alpha \beta\rangle$.

Dem.: Para mostrarmos que (i), (ii), (iii) e (v) valem para $\mathcal{W}(A)$, basta observarmos que $\mathcal{W}(A) \cong \frac{\mathbb{Z}[G]}{\mathcal{K}}$ e usarmos a proposição anterior.

Mostremos então o item (iv). Consideremos o espaço bilinear $(M, b)$ com uma base $\{x, y\}$ tal que, $b(x, y)=0, b(x, x)=\alpha$ e $b(y, y)=\beta$, ou seja $b=\langle\alpha, \beta\rangle$. Então, $b(x+y, x+y)=\alpha+\beta \in A^{\star}$. Como $\alpha+\beta \in A^{\star}$ temos que $(A(x+y), b)$ é um subespaço não singular de $(M, b)$ e de (2.8), $M=A(x+y) \perp(A(x+y))^{\perp}$. Como $(A(x+y))^{\perp}$ é um subespaço não singular de $(M, b)$ unidimensional, existe $z \in M$ $\operatorname{com} b(z, z)=\gamma \in A^{\star} \mathrm{e} b(x+y, z)=0$, ou seja $(A(x+y))^{\perp}=A z$. Assim,

$$
(\alpha\rangle \perp\langle\beta\rangle \simeq\langle\alpha+\beta\rangle \perp\langle\gamma\rangle .
$$

Comparando os determinantes, temos $\alpha \beta \equiv(\alpha+\beta) \gamma \bmod \left(A^{\star}\right)^{2}$. Isto implica que $\alpha \beta(\alpha+\beta)^{-1} \equiv \gamma \bmod \left(A^{\star}\right)^{2}$, ou seja, $\gamma \equiv \alpha \beta(\alpha+\beta) \bmod \left(A^{\star}\right)^{2}$. Logo, de (i) $\langle\gamma\rangle=\langle\alpha \beta(\alpha+\beta)\rangle$. Consequentemente,

$$
\langle\alpha\rangle \perp\langle\beta\rangle \simeq\langle\alpha+\beta\rangle \perp\langle\alpha \beta(\alpha+\beta)\rangle .
$$

Portanto, $\langle\alpha)+\langle\beta\rangle=\langle\alpha+\beta\rangle+\langle\alpha \beta(\alpha+\beta)\rangle$ em $\mathcal{W}(A)$, por (ii).

\subsection{Os ideais primos de $\mathcal{W}(A)$}

Nesta seção caracterizaremos os ideais primos de $\mathcal{W}(A)$ usando o isomorfismo de anéis $\mathcal{W}(A) \cong \frac{\mathbb{Z}[G]}{\mathcal{K}}$, onde $G=A^{\star} / A^{\star^{2}}$ e $\mathcal{K}$ é bem determinado em (3.4), ou seja, 
usaremos o fato que os ideais primos de $\mathcal{W}(A)$ estão em correspondência biunívoca com os ideais primos de $\mathbb{Z}[G]$ que contém $\mathcal{X}$.

Para tanto começaremos determinando todos os ideais primos de $\mathbb{Z}[G]$ e, a seguir aqueles que contém $\mathcal{X}$.

Lema 3.6 Para cada ideal primo $\mathcal{P}$ de $\mathbb{Z}[G]$, temos

(i) Se $\mathcal{P} \cap \mathbb{Z}=\{0\}$, então existe um único homomorfismo de anéis $\phi$ de $\mathbb{Z}[G]$ em $\mathbb{Z}$ com núcleo $\mathcal{P}$.

(ii) Se $\mathcal{P} \cap \mathbb{Z}=p \mathbb{Z}$, onde p é um número inteiro primo, então existe um único homomorfismo de anéis $\psi$ de $\mathbb{Z}[G]$ em $\mathbb{F}_{p}$, com núcleo $\mathcal{P}$, onde $\mathbb{F}_{p}$ denota $o$ corpo finito com p elementos.

Dem.: Consideramos o homomorfismo de anéis sobrejetor $h: \mathbb{Z} \rightarrow \frac{\mathbb{Z}[G]}{\mathcal{P}}$, que é a composição da inclusão $i: \mathbb{Z} \rightarrow \mathbb{Z}[G]$ com a sobrejeção canônica $\pi: \mathbb{Z}[G] \rightarrow \frac{\mathbb{Z}[G]}{\mathcal{P}}$. Assim $\operatorname{Ker}(h)=\mathcal{P} \cap \mathbb{Z}$. Como $\mathcal{P} \cap \mathbb{Z}$ é ideal primo de $\mathbb{Z}$, temos que $\mathcal{P} \cap \mathbb{Z}=\{0\}$ ou $\mathcal{P} \cap \mathbb{Z}=p \mathbb{Z}$, para algum inteiro primo $p$ de $\mathbb{Z}$.

Se $\mathcal{P} \cap \mathbb{Z}=\{0\}$, então $\mathbb{Z} \cong \frac{\mathbb{Z}[G]}{\mathcal{P}}$ e se $\mathcal{P} \cap \mathbb{Z}=p \mathbb{Z}$, então $\frac{\mathbb{Z}}{p \mathbb{Z}} \cong \frac{\mathbb{Z}[G]}{\mathcal{P}}$. Assim estes isomorfismos induzem os homomorfismos $\phi$ e $\psi$ requeridos $\mathrm{e}$, desde que os anéis $\mathbb{Z}$ e $\mathbb{F}_{p}$ não admitem automorfismos não triviais, estes homomorfismos são únicos.

Como $g^{2}=1$ para todo $g \in G$, temos que para todo homomorfismo de anéis $\phi: \mathbb{Z}[G] \rightarrow \mathbb{Z}, \phi(g)^{2}=\phi\left(g^{2}\right)=\phi(1)=1$. Como $\phi(g) \in \mathbb{Z}$, temos que $\phi(g)= \pm 1$. Logo, todo homomorfismo de aneis de $\mathbb{Z}[G]$ em $\mathbb{Z}$ leva $G$ em $\{ \pm 1\}$, ou seja, a restrição $\left.\phi\right|_{G}$, de $\phi$ em $G$ é um caracter do grupo $G$, isto é, um homomorfismo de grupos $\chi$ de $G$ em $\{ \pm 1\}$. Reciprocamente, dado um caracter $\chi: G \rightarrow\{ \pm 1\}$, ele se estende, de maneira única, a um homomorfismo de aneis $\phi_{x}: \mathbb{Z}[G] \rightarrow \mathbb{Z}$, devido a propriedade universal de $\mathbb{Z}[G]$. Assim, para cada homomorfismo $\phi$ existe um único caracter $\chi$ tal que $\phi=\phi_{x}$. 
Agora, seja $p$ um número primo ímpar. $O$ grupo $\{ \pm 1\} \subseteq \mathbb{F}_{p}$, é o subgrupo de todos os elementos de $\left(\mathbb{F}_{p}\right)^{\star}$ de ordem 2 . Logo, a restrição de um homomorfismo de aneis $\psi: \mathbb{Z}[G] \rightarrow \mathbb{F}_{p}$ ao grupo $G$, é também um caracter $\chi: G \rightarrow\{ \pm 1\}$. Assim, existe uma única extensão $\phi_{x}: \mathbb{Z}[G] \rightarrow \mathbb{Z}$ que faz o diagrama abaixo comutar

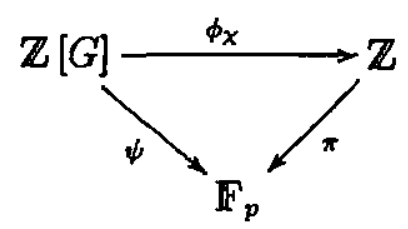

onde $\pi$ é a sobrejeção canônica de $\mathbb{Z}$ em $\mathbb{F}_{p}$.

Consideremos $p=2$. Cada homomorfismo de $\mathbb{Z}[G]$ em $\mathbb{F}_{2}$, leva todo elemento de $G$ em 1. Logo, existe um único homomorfismo de aneis $\psi_{0}: \mathbb{Z}[G] \rightarrow \mathbb{F}_{2}$, que é obtido da composição de $\phi_{x}: \mathbb{Z}[G] \rightarrow \mathbb{Z}$ com a projeção canônica sobre $\mathbb{F}_{2}$, onde $\phi_{x}$ é a extensão de qualquer caracter $\chi: G \rightarrow\{ \pm 1\}$.

Destas observações e do lema (3.6), temos

Proposição 3.7 Para cada ideal primo $\mathcal{P}$ de $\mathbb{Z}[G]$, temos

(i) Se $\mathcal{P} \cap \mathbb{Z}=\{0\}$, então existe um único caracter $\chi$ de $G$, tal que $\mathcal{P}=\mathcal{P}_{\chi}$ é o núcleo do homomorfismo $\phi_{x}: \mathbb{Z}[G] \rightarrow \mathbb{Z}$.

(ii) Se $\mathcal{P} \cap \mathbb{Z}=p \mathbb{Z}, p$ um número primo ímpar, então existe um único caracter $\chi$ de $G$, tal que $\mathcal{P}$ coincide com o conjunto

$$
\mathcal{P}_{\chi, p}:=\left\{z \in \mathbb{Z}[G] ; \phi_{\chi}(z) \equiv 0 \bmod p\right\} .
$$

(iii) Existe um único ideal $\mathcal{P}_{0}$ de $\mathbb{Z}[G]$ com $\mathcal{P}_{0} \cap \mathbb{Z}=2 \mathbb{Z} e$

$$
\mathcal{P}_{0}=\left\{z \in \mathbb{Z}[G] ; \phi_{\chi}(z) \equiv 0 \bmod 2\right\}
$$

para cada caracter $\chi$ de $G$. 
Observação 3.8 É claro que os ideais $\mathcal{P}_{\chi}$, com $\chi$ percorrendo o conjunto dos caracteres de $G$, são todos os ideais primos minimais de $\mathbb{Z}[G]$. Os ideais $\mathcal{P}_{\chi, p} \operatorname{com} \chi$ percorrendo o conjunto dos caracteres de $G, p$ o conjunto dos números primos ímpares e $\mathcal{P}_{0}$ são todos os ideais maximais de $\mathbb{Z}[G]$.

Consideremos agora, os ideais primos de $\mathcal{W}(A) \cong \frac{\mathbb{Z}[G]}{\mathcal{K}}$. Denotemos por $\mathfrak{I}(A)$ o núcleo do homomorfismo de anéis $d_{0}: \mathcal{W}(A) \rightarrow \mathbb{F}_{2}$, definido por

$$
d_{0}([b])=(\operatorname{dim}(b)) \bmod 2
$$

Desde que $\frac{\mathcal{W}(A)}{\mathfrak{I}(A)} \cong \mathbb{F}_{2}$ é corpo, temos que $\mathfrak{I}(A)$ é um ideal maximal de $\mathcal{W}(A)$, chamado o o ideal fundamental de $\mathcal{W}(A)$.

Proposição $3.9 O$ ideal fundamental $\mathfrak{I}(A)$ é o único ideal primo de $\mathcal{W}(A)$ que contém $2\langle 1\rangle=2.1_{\mathcal{W}(A)}$.

Dem.: Resta mostrarmos apenas a unicidade. O item (iii) da proposição anterior garante que $\mathcal{P}_{0}$ é o único ideal primo de $\mathbb{Z}[G]$ que contém $(2) \in G$. Como $\varphi(2)=2\langle 1\rangle$, temos que $\mathfrak{I}(A)$ corresponde ao ideal $\mathcal{P}_{0}$ de $\mathbb{Z}[G]$, na correspondência entre os ideais de $\mathcal{W}(A)$ e os ideais de $\mathbb{Z}[G]$ que contém $\mathcal{K}$. Portanto, a unicidade de $\mathfrak{I}(A)$ decorre da unicidade de $\mathcal{P}_{0}$.

Para uma melhor caracterização dos ideais primos de $\mathcal{W}(A)$, usaremos a noção de assinatura como definida abaixo.

Definição 3.10 Uma assinatura de $A$ é um homomorfismo de anéis de $\mathcal{W}(A)$ em $\mathbb{Z}$. Denotamos por $\mathcal{A} s s(A)$ o conjunto de todas as assinaturas de $A$ e, por $\mathcal{P}_{\sigma}$ o núcleo da assinatura $\sigma$. Dizemos que $A$ é um anel formalmente real se $A$ admite pelo menos uma assinatura, ou seja, se $\mathcal{A} s s(A) \neq \emptyset$. Caso contrário, $A$ é dito ser um anel não formalmente real. 
Assumimos primeiro, que $A$ é um anel formalmente real, ou seja, $\mathcal{A s s}(A) \neq \emptyset$.

Do teorema do isomorfismo para anéis, segue imediatamente que $\frac{\mathcal{W}(A)}{\mathcal{P}_{\sigma}} \cong \mathbb{Z}$, para toda $\sigma \in \mathcal{A s s}(A)$.

Proposição 3.11 Para cada ideal primo $\mathcal{P}$ de $\mathcal{W}(A)$ que não contém $p(1\rangle$, para todo número primo $p$, existe uma única assinatura $\sigma$ de $A$ tal que $\mathcal{P}=\mathcal{P}_{\sigma}$.

Dem.: Seja $\mathcal{P}$ um ideal primo de $\mathcal{W}(A)$ que não contém $p\langle 1\rangle$, para todo número primo $p$. Desde que $\mathcal{W}(A) \cong \frac{\mathbb{Z}[G]}{\mathcal{K}}$, temos que existe $\mathcal{P}^{\prime}=\varphi^{-1}(\mathcal{P})$, ideal primo de $\mathbb{Z}[G]$ tal que $\mathcal{X} \subseteq \mathcal{P}^{\prime}$. Como $p\langle 1\rangle \notin \mathcal{P}$ para todo número primo $p$, o ideal $\mathcal{P}^{\prime}$ é tal que $\mathcal{P}^{\prime} \cap \mathbb{Z}=\{0\}$.

Pelo item (i) da proposição (3.7) existe um único homomorfismo de anéis $\phi_{x}: \mathbb{Z}[G] \rightarrow \mathbb{Z}$, tal que $\operatorname{Ker}\left(\phi_{\chi}\right)=\mathcal{P}^{\prime}$. Mas $\mathcal{X} \subseteq \operatorname{Ker}\left(\phi_{\chi}\right)=\mathcal{P}^{\prime}$. Logo, existe uma única $\sigma \in \mathcal{A} s s(A)$ que faz o seguinte diagrama comutar

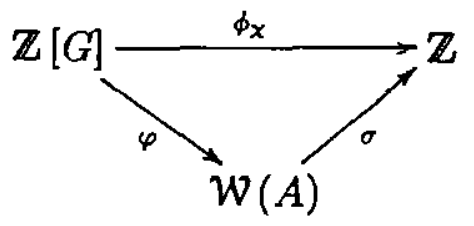

Resta mostrarmos que $\mathcal{P}=\mathcal{P}_{\sigma}$. Desde que $\sigma(\mathcal{P})=\sigma \circ \varphi\left(\mathcal{P}^{\prime}\right)=\phi_{x}\left(\mathcal{P}^{\prime}\right)=0$, temos que $\mathcal{P} \subseteq \mathcal{P}_{\sigma}$. Por outro lado, dado $b \in \mathcal{P}_{\sigma}$, o fato de $\varphi$ ser sobrejetora implica que existe $x \in \mathbb{Z}[G]$ tal que $\varphi(x)=b$. Assim, $\phi_{x}(x)=\sigma \circ \varphi(x)=\sigma(b)=0$, ou seja $x \in \operatorname{Ker}\left(\phi_{x}\right)=\mathcal{P}$. Logo, $\varphi(x)=b \in \mathcal{P}$. Portanto $\mathcal{P}_{\sigma} \subseteq \mathcal{P}$, como queríamos.

Para analisarmos os ideais primos de $\mathcal{W}(A)$ que contém $p\langle 1\rangle$, para algum primo impar $p$, necessitamos das informações sobre o ideal $\mathcal{X}$ contidas no seguinte lema.

Lema 3.12 Para cada caracter $\chi$ de $G$, temos que $\phi_{\chi}(\mathcal{X})=0$ ou $\phi_{\chi}(\mathcal{X}) \subseteq 2^{n} \mathbb{Z}$, para $\operatorname{algum} n \geq 1$. 
Dem.: Para cada caracter $\chi$ de $G$, basta analizarmos a ação de $\phi_{\chi}$ nos geradores de $\mathcal{K}$. Como $\phi_{x}(1)=1$, temos que $\phi_{x}$ leva $(1)+(-1)$ em 0 ou 2.

Seja $z=\sum_{i=1}^{n}\left(\alpha_{i}\right)-\sum_{i=1}^{n}\left(\beta_{i}\right) \in \mathbb{Z}[G]$, tal que $\left(\alpha_{1}, \ldots, \alpha_{n}\right\rangle \simeq\left\langle\beta_{1}, \ldots, \beta_{n}\right\rangle$. Sejam $s$ o número de elementos $\left(\alpha_{i}\right) \in G, 1 \leq i \leq n, \operatorname{com} \phi_{x}\left(\alpha_{i}\right)=-1$ e $t$ o número de elementos $\left(\beta_{i}\right) \in G, 1 \leq i \leq n$, com $\phi_{x}\left(\beta_{i}\right)=1$. Assim,

$$
\phi_{X}(z)=\phi_{X}\left(\sum_{i=1}^{n}\left(\alpha_{i}\right)\right)-\phi_{x}\left(\sum_{i=1}^{n}\left\langle\beta_{i}\right)\right)=-s+(n-s)+t-(n-t)=2\langle t-s) .
$$

Mas $\left\langle\alpha_{1}, \ldots, \alpha_{n}\right\rangle \simeq\left\langle\beta_{1}, \ldots, \beta_{n}\right\rangle$, o que implica que seus determinantes diferem por quadrados, ou seja,

$$
\prod_{i=1}^{n} \alpha_{i} \equiv \prod_{i=1}^{n} \beta_{i} \bmod \left(A^{\star}\right)^{2}, \quad \text { ou } \quad \prod_{i=1}^{n}\left(\alpha_{i}\right)=\prod_{i=1}^{n}\left(\beta_{i}\right) \text { em } \quad G .
$$

Aplicando $\phi_{\chi}$ nesta igualdade e usando que $\phi_{x}\left(\alpha^{2}\right)=1$, para todo $\alpha \in A^{\star}$, obtemos $(-1)^{s}=(-1)^{t}$, isto é, $t-s$ é um número par. Consequentemente, $\phi_{x}(z) \equiv 0 \bmod 4$. Portanto, $\phi_{\chi}(\mathcal{K})=0$ ou $\phi_{\chi}(\mathcal{K}) \subseteq 2^{n} \mathbb{Z}$, para algum $n \geq 1$.

Corolário 3.13 Se $\phi_{x}(\mathcal{K}) \subseteq p \mathbb{Z}$, para algum primo impar p, então $\phi_{X}(\mathcal{K})=0$

Dem.: Imediata.

Proposição 3.14 Seja p um número primo ímpar. Para cada ideal primo $\mathcal{P}$ de $\mathcal{W}(A)$ com $p(1\rangle \in \mathcal{P}$, existe uma única assinatura $\sigma$ de A tal que $\mathcal{P}$ coincide com $o$ conjunto

$$
\mathcal{P}_{\sigma, p}=\{b \in \mathcal{W}(A) ; \sigma(b) \equiv 0 \bmod p\}
$$

Dem.: Seja $\mathcal{P}$ um ideal primo de $\mathcal{W}(A) \operatorname{com} p\langle 1\rangle \in \mathcal{P}$. Novamente pelo isomorfismo $\mathcal{W}(A) \cong \frac{\mathbb{Z}[G]}{\mathcal{K}}$ temos que existe $\mathcal{P}^{\prime} \subseteq \mathbb{Z}[G]$ tal que $\mathcal{K} \subseteq \mathcal{P}^{\prime}$ e $\varphi\left(\mathcal{P}^{\prime}\right)=\mathcal{P}$. Como 
$p\langle 1\rangle \in \mathcal{P}$, temos que $\mathcal{P}^{\prime} \cap \mathbb{Z}=p \mathbb{Z}$. Por (3.7), temos que existe um único caracter $\chi$ de $G$, tal que $\mathcal{P}^{\prime}=\mathcal{P}_{x, p}=\left\{z \in \mathbb{Z}[G] ; \phi_{\chi}(z) \equiv 0 \bmod p\right\}$.

Do fato que $\mathcal{K} \subseteq \mathcal{P}^{\prime}=\mathcal{P}_{x, p}$, segue que $\phi_{X}(\mathcal{K})=p \mathbb{Z}$. Assim de (3.13) temos que $\phi_{\chi}(\mathcal{K})=0$. Logo $\mathcal{K} \subseteq \operatorname{Ker}\left(\phi_{\chi}\right)$ e, consequentemente, existe uma única $\sigma \in \mathcal{A} s s(A)$ que faz o diagrama abaixo comutar

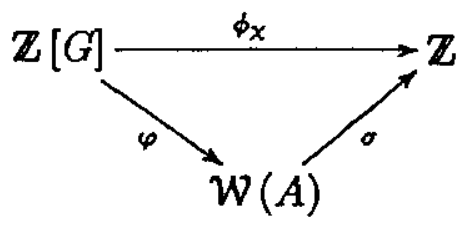

Mostremos agora que $\mathcal{P}=\mathcal{P}_{\sigma, p}$. Dado $b \in \mathcal{P}$, existe $x \in \mathcal{P}^{\prime}$ tal que $\varphi(x)=b$, pois $\mathcal{P}^{\prime}=\varphi^{-1}(\mathcal{P})$. Da definição de $\mathcal{P}^{\prime}$, temos que $\phi_{x}(x) \equiv 0 \bmod p$. Assim, $\sigma(b)=$ $\sigma \circ \varphi(x)=\phi_{x}(x) \equiv 0 \bmod p$, ou seja, $b \in \mathcal{P}_{\sigma, p}$, o que mostra que $\mathcal{P} \subseteq \mathcal{P}_{\sigma, p}$. Seja agora $b \in \mathcal{P}_{\sigma, p}$. Segue da sobrejetividade de $\varphi$ que existe $x \in \mathbb{Z}[G]$ tal que $\varphi(x)=b$. Assim, $\phi_{x}(x)=\sigma \circ \varphi(x)=\sigma(b) \equiv 0 \bmod p$, ou seja, $x \in \mathcal{P}^{\prime}$. Logo, $b=\varphi(x) \in \mathcal{P}$. Portanto, $\mathcal{P}_{\sigma, p}=\mathcal{P}$, como queríamos.

De (3.7) e da correspondência entre os ideais de $\mathcal{W}(A)$ com os ideais de $\mathbb{Z}[G]$, que contém $\mathcal{K}$, concluimos que se $\mathcal{A s s}(A) \neq \emptyset$ então $\mathcal{P}_{\sigma}, \mathcal{P}_{\sigma, p}$, para cada $\sigma \in \mathcal{A} s(A)$ e todo número primo ímpar $p$, e $\mathfrak{I}(A)$ são todos os ideais primos de $\mathcal{W}(A)$.

Teorema 3.15 Se A é um anel semilocal formalmente real, então:

(i) Os $\mathcal{P}_{\sigma}$, com $\sigma \in \mathcal{A s s}(A)$, são todos os ideais primos minimais de $\mathcal{W}(A)$.

(ii) Os $\mathcal{P}_{\sigma, p}$ com $\sigma \in \mathcal{A} s s(A)$ e p um número primo impar, e $\mathfrak{I}(A)$ são todos os ideais primos maximais de $\mathcal{W}(A)$.

(iii) Cada $\mathcal{P}_{\sigma, p}$ contém um único ideal primo minimal, a saber $\mathcal{P}_{\sigma}$.

(iv) $\mathfrak{I}(A)$ contém todos os ideais primos minimais. 
Dem.: Resta apenas mostrarmos (iii) e (iv). $\mathrm{Em} \mathbb{Z}$ existe um único ideal maximal que contém $p$. Então, devido ao isomorfismo $\mathcal{W}(A) / \mathcal{P}_{\sigma} \cong \mathbb{Z}$, existe um único ideal maximal de $\mathcal{W}(A)$ que contém $p\langle 1\rangle$ e $\mathcal{P}_{\sigma}$, que é claramente $\mathcal{P}_{\sigma, p}$. Suponhamos que exista um ideal primo minimal $\mathcal{P}_{\psi}$ de $\mathcal{W}(A)$, tal que $\mathcal{P}_{\psi}$ também esta contido em $\mathcal{P}_{\sigma, p}$. Como $\mathcal{P}_{\sigma, p}$ contém $\mathcal{P}_{\psi}$ e $p\langle 1\rangle$ temos que $\mathcal{P}_{\sigma, p}=\mathcal{P}_{\psi, p}$. Segue então de (3.14) que $\sigma=\psi$. Portanto $\mathcal{P}_{\sigma}=\mathcal{P}_{\psi}$, o que mostra (iii).

Agora, queremos mostrar que $\mathcal{P}_{\sigma} \subseteq \mathfrak{I}(A)$, para cada $\sigma \in \mathcal{A} s s(A)$. Para tanto, seja $b=\left\langle\alpha_{1}, \ldots, \alpha_{n}\right\rangle \in \mathcal{P}_{\sigma}$. Como $\sigma\left(\left\langle\alpha_{i}\right\rangle\right)= \pm 1$, para $1 \leq i \leq n$ e, $\sigma(b)=0$, temos que $n$ tem que ser par e para metade dos índices $i=1, \ldots, n, \sigma\left(\left\langle\alpha_{i}\right\rangle\right)=1$, e para a outra metade $\sigma\left(\left\langle\alpha_{i}\right\rangle\right)=-1$. Portanto $\mathcal{P}_{\sigma} \subseteq \mathfrak{I}(A)$, como queríamos.

Para o caso em que $A$ é um anel não formalmente real temos

Teorema 3.16 $O$ anel $A$ é não formalmente real se, e somente se $\mathfrak{I}(A)$ é o único ideal primo de $\mathcal{W}(A)$.

Dem.: Da descrição dos ideais de $\mathbb{Z}[G]$, temos claramente que se $\mathcal{A} s s(A)=\emptyset$, então $\mathfrak{I}(A)$ é o único ideal primo de $\mathcal{W}(A)$. Reciprocamente se $\mathfrak{I}(A)$ é o único ideal primo de $\mathcal{W}(A)$, então $\mathfrak{I}(A)$ é o nilradical de $\mathcal{W}(A)$, isto é, o conjunto de todos os elementos nilpotentes de $\mathcal{W}(A)$. Em particular, $2\langle 1\rangle \in \mathfrak{I}(A)$ é nilpotente. Assim existe $n \geq 1$ tal que $2^{n}\langle 1)=(2(1\rangle)^{n}=0 \mathrm{em} \mathcal{W}\langle A)$, o que implica que $\langle 1\rangle \in \mathcal{W}(A)$ é um elemento de torção. Como $\mathbb{Z}$ é um anel livre de torção e assumimos que todo homomorfismo de anéis leva elemento identidade em elemento identidade, temos que não existe homomorfismo de anéis de $\mathcal{W}(A)$ em $\mathbb{Z}$, ou seja, $A$ é não formalmente real.

Como uma consequência imediata deste teorema temos:

Corolário 3.17 Se A é não formalmente real, então os divisores de zero de $\mathcal{W}(A)$ tem dimensão par, isto é, são representados por um espaço bilinear de dimensão par. 
Dem.: De (3.16) temos que $\mathcal{W}(A)$ é um anel local com único ideal maximal $\mathfrak{I}(A)$. Logo, os divisores de zero, que não são inversíveis, estão em $\mathfrak{I}(A)$.

\section{3 $\quad \mathcal{N} i l(\mathcal{W}(A))$ e $\mathcal{W}_{t}(A)$}

Nesta seção apresentamos alguns resultados sobre os elementos de torção, os elementos nilpotentes e os divisores de zero do anel de Witt de $A$.

Desde que $\mathcal{W}(A)$ é um anel comutativo com elemento identidade $\langle 1\rangle$, o conjunto dos elementos nilpotentes de $\mathcal{W}(A)$ formam o nilradical de $\mathcal{W}(A)$, que denotaremos por $\mathcal{N} i l(\mathcal{W}(A))$. O ideal dos elementos de torção de $\mathcal{W}(A)$ será denotado por $\mathcal{W}_{t}(A)$.

Em um anel $R$, um elemento de torção $x \in R$ é dito ter $p$-torção, $p \in \mathbb{Z}$ um número primo, se $x$ é anulado por uma potência de $p$. Dentre os resultados apresentados nesta seção, mostraremos que o anel $\mathcal{W}(A)$ tem somente 2-torção.

Ao contrário da seção anterior, assumiremos primeiramente que $A$ é um anel semilocal não formalmente real. Neste caso, como conseqüência imediata do teorema (3.16) e sua demonstração, temos

Teorema 3.18 Se A é um anel semilocal não formalmente real, então

(i) $\mathcal{N i l}(\mathcal{W}(A))=\mathfrak{I}(A)$.

(ii) $\mathcal{W}_{t}(A)=\mathcal{W}(A)$

(iii) $\mathcal{W}(A)$ tem somente 2 -torção.

Dem.: Imediata.

Agora seja $A$ um anel semilocal formalmente real. Desde que os $\mathcal{P}_{\sigma}$, com $\sigma$ em $\mathcal{A} s s(A)$, são todos os ideais primos minimais de $\mathcal{W}(A)$, temos 
Proposição 3.19 Um elemento $b \in \mathcal{W}(A)$ é nilpotente se, e somente se $\sigma(b)=0$ para toda $\sigma \in \mathcal{A}$ ss $(A)$, isto é, $\mathcal{N i l}(\mathcal{W}(A))=\bigcap_{\sigma \in \mathcal{A} s s(A)} \operatorname{Ker}(\sigma)$.

Dem.: Imediata.

Para o ideal de torção temos

Proposição 3.20 Se A é um anel semilocal formalmente real, então

$$
\mathcal{W}_{t}(A)=\mathcal{N i l}(\mathcal{W}(A))
$$

Dem.: Sejam $b \in \mathcal{W}_{t}(A)$ e $n \in \mathbb{N}, n \geq 1$, tal que $n b=0$. Então, para cada $\sigma \in \mathcal{A} s s(A)$, temos $\sigma(n b)=n \sigma(b)=0$, o que implica que $\sigma(b)=0$. Assim, $b$ pertence a $\bigcap_{\sigma \in \mathcal{A} s(A)} \operatorname{Ker}(\sigma)=\mathcal{N i l}(\mathcal{W}(A))$, o que mostra que $\mathcal{W}_{t}(A) \subseteq \mathcal{N i l}(\mathcal{W}(A))$. Reciprocamente, dado $b=\left\langle\alpha_{1}, \ldots, \alpha_{n}\right\rangle \in \mathcal{N} i l(\mathcal{W}(A))$; consideremos $H$ o subgrupo de $G$ gerado por $\left\{\left(\alpha_{1}\right), \ldots,\left(\alpha_{n}\right)\right\}$. Então, desde que todo elemento de $G$ tem ordem 2 , temos que $H$ é um subgrupo finito de $G$ e $b$ está no subanel $R$ de $\mathcal{W}(A)$ isomorfo à $\frac{\mathbb{Z}[H]}{(\mathcal{K} \cap \mathbb{Z}[H])}$. Usando o teorema de Maschke, ver (3.6) em [13], temos que o anel de grupo $\mathbb{Q}[H] \cong \mathbb{Q} \otimes \mathbb{Z}[H]$ é semi-simples, ou seja, $\mathcal{N} i l(\mathbf{Q}[H])=\{0\}$.

Considerando que $R \cong \mathbb{Z}[H]$, implica que $\mathbb{Q} \otimes \mathbb{Z}[H] \cong \mathbf{Q} \otimes R$, temos por (3.1.b) de [13] que $\mathcal{N i l}(\mathbf{Q} \otimes R)=\{0\}$.

Mas, $1 \otimes b \in \mathbb{Q} \otimes \mathcal{N i l}(R) \subseteq \mathcal{N} i l\langle\mathbf{Q} \otimes R)=\{0\}$. Também identificando $\mathbf{Q} \otimes R$ com $\mathrm{T}^{-1}(\mathbb{Z}) \otimes R \cong \mathrm{T}^{-1}(R)$, onde $T=\mathbb{Z}-\{0\}$, temos $0=1 \otimes b=\frac{b}{1} \in \mathrm{T}^{-1}(R)$, o que é equivalente a existir $n \in \mathrm{T}, n \geq 1$, tal que $n b=0 \mathrm{em} R$. Assim, $b \in R_{t} \subseteq \mathcal{W}_{t}(A)$, o que mostra a proposição.

Das duas últimas proposições, deduzimos imediatamente o Princípio Local-Global de Pfister para espaços bilineares sobre um anel semilocal formalmente real. 
Teorema 3.21 (Princípio Local-Global de Pfister) Seja $A$ um anel semilocal formalmente real. Então uma forma bilinear $b$ representa um elemento de torção em $\mathcal{W}(A)$ se, e somente se $\sigma(b)=0$, para toda $\sigma \in \mathcal{A} s s(A)$.

Dem.: Imediata.

Mostremos agora que também no caso em que $A$ é formalmente real $\mathcal{W}(A)$ tem somente 2-torção.

Teorema 3.22 Se A é um anel semilocal formalmente real, então $\mathcal{W}(A)$ tem somente 2-torção.

Dem.: Seja $b=\left\langle\alpha_{1}, \ldots, \alpha_{n}\right\rangle \in \mathcal{W}_{t}(A)$. Desde que $\mathcal{W}_{t}(A)=\mathcal{N} i l(\mathcal{W}(A))$, temos que $b \in \mathcal{N} i l(\mathcal{W}(A))$ e, como na demonstração da proposição anterior, $b$ está no subanel $R$ de $\mathcal{W}(A)$ isomorfo à $\frac{\mathbb{Z}[H]}{(\mathcal{K} \cap \mathbb{Z}[H])}$, onde $H$ é o subgrupo de $G$ gerado por $\left\{\left(\alpha_{1}\right), \ldots,\left(\alpha_{n}\right)\right\}$. Agora, para mostrarmos que $b$ é 2-torção em $\mathcal{W}(A)$, é suficiente mostrarmos que o anel $R$ não tem $p$-torção, para todo número primo ímpar $p$.

Seja $p$ um número primo ímpar qualquer. Desde que $R \cong \frac{\mathbb{Z}[H]}{(\mathcal{K} \cap \mathbb{Z}[H])}$, temos que $\frac{R}{p R} \cong \frac{\mathbb{F}_{p}[H]}{\mathcal{K}^{\prime}}$, para algum subgrupo $\mathcal{K}^{\prime}$ de $\mathbb{F}_{p}[H]$.

Como $H \subseteq G$, temos que os elementos de $H$ tem ordem 2. Desde que $\mathbb{F}_{p}$ tem característica $p$ um primo ímpar, novamente pelo teorema de Maschke, obtemos que $R / p R$ é um anel semi-simples, ou seja, $\mathcal{N i l}(R / p R)=\{0\}$.

Se $b_{0} \in R$ é nilpotente, então $\overline{b_{0}} \in \mathcal{N} i l(R / p R)=\{0\}$, ou seja, existe $b_{1} \in R$ tal que $b_{0}=p b_{1}$. Isto, e o fato que $\mathcal{W}_{t}(A)=\mathcal{N} i l(\mathcal{W}(A))$, mostra que o ideal de torção $R_{t}$ de $R$ é divisível por cada número primo ímpar, isto é, $R_{t}=p R_{t}$ para cada número primo ímpar $p$.

Se $b_{0} \in R$ tem $p$-torção, entao $b_{0} \in R_{t}=p R_{t}$, ou seja, $b_{0}=p b_{1}$, com $b_{1}$ em $R_{t}$ que também tem $p$-torção, o que implica que $b_{1}=p b_{2}$, para algum $b_{2} \in R_{t} \mathrm{e}$ consequentemente, $b_{0}=p^{2} b_{2}$. Assim, para cada inteiro $n \geq 0$, existe $b_{n} \in R_{t}$, onde $b_{n}$ tem $p$-torção e $b_{0}=p^{n} b_{n}$. 
Mas $R$ é um grupo abeliano finitamente gerado, então pela decomposição dos $\mathbb{Z}$-módulos finitamente gerado, temos que $R_{t}$ é um grupo finito. Logo, existe $N>0$ tal que $p^{N} b^{\prime}=0$, para todo $b^{\prime} \in R_{t}$ com $p$-torção. Logo $b_{0}=p^{N} b_{N}=0 \mathrm{em} R$. Como $p$ é um número primo ímpar qualquer, temos que $R$ não tem $p$-torção, para todo número primo ímpar $p$, como queríamos.

Como consequência do teorema anterior deduzimos que também no caso em que $A$ é formalmente real, os divisores de zero de $\mathcal{W}(A)$ tem dimensão par.

Corolário 3.23 Os divisores de zero de $\mathcal{W}(A)$ tem dimensão par.

Dem.: Em [07], página 3, temos que o conjunto dos divisores de zero de $\mathcal{W}(A)$ é uma união de ideais primos. Suponhamos que $p\langle 1\rangle$ é um divisor de zero, para algum número primo ímpar $p$. Então existe $b \in \mathcal{W}(A), b \neq 0$, tal que $p\langle 1\rangle \otimes b=0$ em $\mathcal{W}(A)$, o que implica que $b$ tem $p$-torção em $\mathcal{W}(A)$. Mas, do teorema anterior temos que $\mathcal{W}(A\rangle$ tem somente 2 -torção. Assim, $p\langle 1\rangle$ não é divisor de zero para nenhum primo ímpar $p$. Assim, na união dos ideais primos que compoem os divisores de zero, não aparece ideais primos da forma $\mathcal{P}_{\sigma, p}, \operatorname{com} \sigma \in \mathcal{A} s s(A)$ e $p$ um número primo ímpar, ou seja, de (3.15), temos que cada ideal primo que aparece na união é minimal ou $\mathfrak{I}(A)$. Desde que $\mathcal{P}_{\sigma} \subseteq \mathfrak{I}(A)$, para todo $\sigma \in \mathcal{A} s s(A)$, o resultado segue. 


\section{Capítulo 4}

\section{Ideais Primários no Anel de Witt}

Neste capítulo apresentaremos uma caracterização dos ideais primários de $\mathcal{W}(A)$ cujos radicais estão caracterizados em (3.15) e (3.16). Apresentaremos também condições necessárias e suficientes para que todo ideal de $\mathcal{W}(A)$ admita uma decomposição primária, bem como alguns resultados sobre ideais decomponíveis contendo uma forma de dimensão ímpar.

No restante deste trabalho, para simplificar a notação, denotaremos as operações $\perp \mathrm{e} \otimes \mathrm{em} \mathcal{W}(A)$ por $+\mathrm{e} \cdot$ respectivamente. Denotaremos também por $b^{\mathrm{n}}$ o elemento $b \otimes b \otimes \ldots \otimes b$ (n-vezes) em $\mathcal{W}(A)$. Mais ainda, salvo menção em contrário, todas as igualdades envolvendo espaços bilineares são igualdades de elementos de $\mathcal{W}(A)$.

\subsection{Ideais Primários de $\mathcal{W}(A)$}

Nesta seção apresentaremos a caracterização dos ideais $\mathcal{P}$-primários para cada tipo de ideal primo caracterizado em (3.15) e (3.16), onde $A$ é um anel semilocal. Iniciaremos com a caracterização dos ideais $\mathfrak{I}(A)$-primários, onde $\mathfrak{I}(A)$ é o ideal fundamental de $\mathcal{W}(A)$, ou seja $\mathfrak{I}(A)=\{b \in \mathcal{W}(A) ; \operatorname{dim}(b)$ é par $\}$. Para tanto, necessitaremos dos seguintes resultados auxiliares

Lema 4.1 $O$ ideal fundamental $\mathfrak{I}(A)$ é aditivamente gerado pelo conjunto 
$\left\{\langle 1, \alpha\rangle \in \mathcal{W}(A) ; \alpha \in A^{*}\right\}$

Dem.: Dado $b \in \mathfrak{I}(A)$, de (3.2), podemos escrever $b=\left\langle\alpha_{1}, \ldots, \alpha_{2 n}\right\rangle$, com $\alpha_{i} \in A^{*}, \quad i=1, \ldots, 2 n$. Desde que $\langle 1,-1\rangle=0$ em $\mathcal{W}(A)$, temos que $b=\prod_{i=1}^{n}\left\langle 1, \alpha_{i}\right\rangle-\sum_{i=n+1}^{2 n}\left\langle 1,-\alpha_{i}\right\rangle$, o que mostra o lema.

Lema 4.2 Para cada $\alpha \in A^{*}$, temos que $\langle 1, \alpha\rangle^{k+1}=2^{k}\langle 1, \alpha\rangle$ em $\mathcal{W}(A)$, para todo inteiro $k \geq 1$.

Dem.: A demonstração será feita usando indução sobre $k$. Se $k=1$, então $\langle 1, \alpha\rangle^{2}=$ $\langle 1, \alpha\rangle \otimes\langle 1, \alpha\rangle=\left\langle 1, \alpha, \alpha, \alpha^{2}\right\rangle=\langle 1, \alpha, 1, \alpha\rangle=2\langle 1, \alpha\rangle$. Suponhamos agora que o resultado vale para $k-1$, ou seja, $\langle 1, \alpha\rangle^{k}=2^{k-1}\langle 1, \alpha\rangle$. Assim

$$
\langle 1, \alpha\rangle^{k+1}=\langle 1, \alpha\rangle^{k} \cdot\langle 1, \alpha\rangle=2^{k-1}\langle 1, \alpha\rangle \cdot\langle 1, \alpha\rangle=2^{k}\langle 1, \alpha\rangle,
$$

o que conclui a demonstração.

Teorema 4.3 Seja $\partial \subseteq \mathcal{W}(A)$ um ideal. Então $\partial$ é $\mathfrak{I}(A)$-primário se, e somente se $2^{k}\langle 1\rangle \in \mathcal{J}$, para algum inteiro positivo $k$.

Dem.: Se $\partial$ é $\mathfrak{I}(A)$-primário então $2^{k}\langle 1\rangle=(2\langle 1\rangle)^{k} \in J$, para algum inteiro $k \geq 1$, pois $2\langle 1\rangle=\langle 1,1\rangle \in \mathfrak{I}(A\rangle=r(\partial)$.

Reciprocamente, suponhamos que $2^{k}\langle 1\rangle \in \partial$, para algum inteiro $k \geq 1$. Assim, $2\langle 1\rangle \in r(\nexists)$ e, como de (3.9) $\mathfrak{I}(A\rangle$ é o único ideal maximal de $\mathcal{W}(A)$ que contém $2\langle 1\rangle$, obtemos que $r(\mathcal{J}) \subseteq \mathfrak{I}(A)$. Para provarmos que $\mathfrak{I}(A) \subseteq r(\mathcal{J})$, usando o lema (4.1) é suficiente mostrarmos que $\langle 1, \alpha\rangle \in r(\mathcal{J})$, para todo $\alpha \in A^{*}$. Dado $\alpha \in A^{*}$, desde que $2^{k}\langle 1\rangle \in \partial$ para algum $k \geq 1$ e $2^{k}\langle 1, \alpha\rangle=2^{k}\langle 1)+2^{k}\langle 1\rangle \cdot\langle\alpha\rangle$, temos do lema (4.2) que $\langle 1, \alpha\rangle^{k+1} \in \mathcal{J}$, ou seja, $\langle 1, \alpha\rangle \in r(\mathcal{J})$. Assim, $r(\mathcal{J}\rangle=\mathfrak{I}(A)$ e de (4.2) de [01], temos que $\partial$ é $\mathfrak{I}(A)$-primário. 
Corolário 4.4 Se A é não formalmente real então todo ideal de $\mathcal{W}(A)$ é $\mathfrak{I}(A)$-primário.

Dem.: Da demonstração de (3.16) temos que $2\langle 1\rangle$ é um elemento nilpotente de $\mathcal{W}(A)$. Assim, existe um inteiro $k \geq 1$ tal que $(2\langle 1\rangle)^{k}=2^{k}\langle 1\rangle=0$, consequentemente $2^{k}\langle 1\rangle \in \mathcal{J}$, para cada ideal $\partial$ de $\mathcal{W}(A)$. Agora o resultado segue de (4.3).

$\mathrm{O}$ próximo passo é analizarmos os ideais $\mathcal{P}$-primários correspondentes aos ideais primos $\mathcal{P}$ de $\mathcal{W}(A)$ distintos de $\mathfrak{I}(A)$. De (3.15) e (3.16) temos que tais ideais primos existem se, e somente se $\mathcal{A} s s(A) \neq \emptyset$. Portanto, no que segue, assumiremos que $A$ é um anel semilocal formalmente real. Para os ideais primos minimais temos:

Teorema 4.5 Sejam $\mathfrak{g} \subseteq \mathcal{W}(A)$ um ideal e $\sigma \in \mathcal{A} s s(A)$. Então $\partial$ é $\mathcal{P}_{\sigma}$-primário se, e somente se $\partial=\mathcal{P}_{\sigma}$.

Dem.: Claramente $\mathcal{P}_{\sigma}$ é $\mathcal{P}_{\sigma}$-primário. Reciprocamente, seja $g \subseteq \mathcal{W}(A)$ um ideal $\mathcal{P}_{\sigma}$-primário. Suponhamos que $\mathfrak{J} \neq \mathcal{P}_{\sigma}$. Como $\mathscr{J} \subseteq r(\mathcal{J})=\mathcal{P}_{\sigma} \subseteq \mathfrak{I}(A)$, obtemos de (4.1) que existe $\alpha \in A^{*}$ tal que $\langle 1,-\alpha\rangle \in \mathcal{P}_{\sigma}-g$. Como $\langle 1,-\alpha\rangle \in \mathcal{P}_{\sigma}$ então $\langle 1,-\alpha\rangle^{m} \in \mathcal{J}$, para algum inteiro $m>1$. Mas, de (4.2), temos que $\langle 1,-\alpha\rangle^{m}=$ $2^{(m-1)}\langle 1,-\alpha\rangle=2^{m-1}\langle 1\rangle \cdot\langle 1,-\alpha\rangle$. Agora, como $\partial$ é um ideal primário de $\mathcal{W}(A) \mathrm{e}$ $\langle 1,-\alpha\rangle \notin \partial$, temos que existe um inteiro $s \geq 1$ tal que $\left(2^{m-1}\langle 1\rangle\right\rangle^{s} \in J$, ou seja, $2^{k}\langle 1\rangle \in \mathcal{J}$ para algum inteiro positivo $k$. Mas, isto é uma contradição, pois $\partial \subseteq \mathcal{P}_{\sigma} \mathbf{e}$ $\sigma\left(2^{k}\langle 1\rangle\right)=2^{k} \neq 0$. Logo $\partial=\mathcal{P}_{\sigma}$ como queríamos.

Finalmente, caracterizaremos os ideais $\mathcal{P}$-primários onde $\mathcal{P}$ é um ideal primo maximal de $\mathcal{W}(A)$ distinto de $\mathfrak{I}(A)$. Mostraremos, neste caso, que os ideais $\mathcal{P}$-primários são exatamente as potências de $\mathcal{P}$.

Dado $\mathcal{P}_{\sigma, p} \in \operatorname{Spec}(\mathcal{W}(A)\rangle, \sigma \in \mathcal{A} s s(A)$ e $p$ um número primo ímpar, para cada inteiro $i \geq 1$, denotaremos por $\mathcal{P}_{\sigma, p^{i}}$ o ideal de $\mathcal{W}(A)$

$$
\mathcal{P}_{\sigma, p^{i}}=\left\{b \in \mathcal{W}(A) ; \sigma(b) \equiv 0 \bmod p^{i}\right\} .
$$


Com esta notação temos

Lema 4.6 Para cada $i \geq 1,\left(\mathcal{P}_{\sigma, p}\right)^{i}=\mathcal{P}_{\sigma, p^{i}}$.

Dem.: É fácil ver que $\left(\mathcal{P}_{\sigma, p}\right)^{i} \subseteq \mathcal{P}_{\sigma, p^{i}}$. Desde que $\mathcal{P}_{\sigma} \subseteq \mathfrak{I}(A)$, de (4.1) e da definição de $\mathcal{P}_{\sigma}$, obtemos que $\mathcal{P}_{\sigma}$ é aditivamente gerado por elementos da forma $\langle 1, \alpha)$, com $\alpha \in A^{*}$ tal que $\sigma(\langle\alpha\rangle)=-1$. Para um tal gerador $\langle 1, \alpha\rangle \in \mathcal{P}_{\sigma}$ e $s=\frac{(p-1)}{2} \in \mathbb{Z}$, temos que

$$
\langle 1, \alpha\rangle=\langle 1, \alpha\rangle \cdot(\langle 1\rangle \perp s\langle 1,-\alpha))^{i}
$$

para todo inteiro positivo $i$, pois $\langle 1, \alpha\rangle \cdot\langle 1,-\alpha\rangle=0$ em $\mathcal{W}(A)$. Mas $b=\langle 1\rangle \perp s\langle 1,-\alpha\rangle \in \mathcal{P}_{\sigma, p}$, pois $\sigma(b)=p$. Assim, $\langle 1, \alpha\rangle \in\left(\mathcal{P}_{\sigma, p}\right)^{i}$, o que mostra que $\mathcal{P}_{\sigma} \subseteq\left(\mathcal{P}_{\sigma, p}\right)^{i}$. Desde que $p^{i}\langle 1\rangle \in\left(\mathcal{P}_{\sigma, p}\right)^{i}$, obtemos

$$
\mathcal{P}_{\sigma, p^{i}}=\mathcal{P}_{\sigma}+p^{i}\langle 1\rangle \cdot \mathcal{W}(A) \subseteq\left(\mathcal{P}_{\sigma, p}\right)^{i}
$$

o que mostra o lema.

Teorema 4.7 Sejam $\partial \subseteq \mathcal{W}(A)$ um ideal, p um número primo impar e $\sigma \in \mathcal{A} s s(A)$. Então $\partial$ é $\mathcal{P}_{\sigma, p}$-primário se, e somente se $\partial=\left(\mathcal{P}_{\sigma, p}\right)^{i}$, para algum inteiro $i \geq 1$.

Dem.: Se $\partial=\left(\mathcal{P}_{\sigma, p}\right)^{i}$, para algum $i \geq 1$, então do lema anterior temos que $\partial=\mathcal{P}_{\sigma, p^{i}}$. Seja $s: \mathcal{W}(A) \longrightarrow \mathbb{Z} / p^{i} \mathbb{Z}$ o homomorfismo sobrejetor de anéis obtido pela composta da assinatura $\sigma$ com a projeção canônica $\pi: \mathbb{Z} \longrightarrow \mathbb{Z} / p^{i} \mathbb{Z}$, ou seja, $s(b)=\sigma(b)+p^{i} \mathbb{Z}$, para todo $b \in \mathcal{W}(A)$. Então $\frac{\mathcal{W}(A)}{\operatorname{Ker}(s)} \cong \frac{\mathbb{Z}}{p^{i} \mathbb{Z}}$ que é um anel onde cada divisor de zero é nilpotente o que mostra que $\operatorname{Ker}(s)$ é um ideal primário de $\mathcal{W}(A)$. Mas $\operatorname{Ker}(s)=$ $\{b \in \mathcal{W}(A) ; s(b)=0\}=\mathcal{P}_{\sigma, p^{i}}$. Logo $\mathcal{P}_{\sigma, p^{i}}$ é um ideal primário de $\mathcal{W}(A)$ para cada $\sigma \in \mathcal{A} s s(A), p$ número primo ímpar e $i \geq 1$ inteiro. Mais ainda, do lema anterior, temos que $r\left(\mathcal{P}_{\sigma, p^{i}}\right)=r\left(\left(\mathcal{P}_{\sigma, p}\right)^{i}\right)=\mathcal{P}_{\sigma, p}$, ou seja $\mathcal{P}_{\sigma, p^{i}}$ é $\mathcal{P}_{\sigma, p^{-p}}$ primário.

Reciprocamente, seja $\partial \subseteq \mathcal{W}(A)$ um ideal $\mathcal{P}_{\sigma, p}$-primário. Neste caso, temos que $\mathcal{P}_{\sigma} \subseteq \mathcal{J}$. De fato, dado $\alpha \in A^{*}$, com $\sigma(\langle\alpha\rangle)=-1$, temos que 
$b=\langle 1\rangle \perp s\langle 1,-\alpha\rangle \in \mathcal{P}_{\sigma, p}$, para $s=\frac{p-1}{2}$. Então, $b^{m} \in \mathfrak{J}$ para algum inteiro positivo $m$, pois $\mathcal{P}_{\sigma, p}=\tau(\mathfrak{J})$. Agora, como na demonstração do lema (4.6), $\langle 1, \alpha\rangle=$ $(1, \alpha\rangle \cdot b^{m} \in \mathcal{J}$, o que mostra que de fato $\mathcal{P}_{\sigma} \subseteq \mathcal{J}$, quando $\mathcal{\partial}$ é $\mathcal{P}_{\sigma, p}$-primário.

Desde que $\mathcal{P}_{\sigma} \subseteq \mathcal{J}$, temos uma sobrejeção canônica $\pi: \frac{\mathcal{W}(A)}{\mathcal{P}_{\sigma}} \longrightarrow \frac{\mathcal{W}(A)}{\mathfrak{J}}$. Mais ainda, dado $\sigma \in \mathcal{A s s}(A)$, temos que $\sigma: \mathcal{W}(A) \longrightarrow \mathbb{Z}$ é um homomorfismos sobrejetor de anéis que induz um isomorfismo $\bar{\sigma}: \frac{\mathcal{W}(A)}{\mathcal{P}_{\sigma}} \rightarrow \mathbb{Z}$. Assim, obtemos uma seqüência de homomorfismos de anéis

$$
\mathbb{Z} \longrightarrow \frac{\mathcal{W}(A)}{\mathcal{P}_{\sigma}} \longrightarrow \stackrel{\mathcal{W}(A)}{\partial}
$$

onde $\tau$ é o isomorfismo inverso de $\bar{\sigma}$, isto é, $\tau(n)=n\langle 1\rangle+\mathcal{P}_{\sigma}$, para todo $n \in \mathbf{Z}$. Claramente $\pi \circ \tau$ é sobrejetor e, com isso, temos que $\frac{\mathcal{W}(A)}{\partial} \cong \frac{\mathbb{Z}}{\operatorname{Ker}(\pi \circ \tau)}$.

Usando o fato que um ideal não nulo $\partial$ é um ideal primário de $\mathcal{W}(A)$ se, e somente se os divisores de zero de $\frac{\mathcal{W}(A)}{\mathfrak{J}}$ são nilpotentes, juntamente com o isomorfismo acima, obtemos que $\operatorname{Ker}(\pi \circ \tau\rangle$ é um ideal primário de $\mathbb{Z}$. Mais ainda, como $p\langle 1\rangle \in \mathcal{P}_{\sigma, p}=$ $\tau(\mathfrak{J})$, temos que $p^{k}\langle 1\rangle \in \mathcal{J}$ para algum inteiro positivo $k$ e, consequentemente, $p^{k}$ está em $\operatorname{Ker}(\pi \circ \tau)$ o que mostra que $\operatorname{Ker}(\pi \circ \tau)$ é um ideal primário de $\mathbb{Z}$ que contém uma potência de $p$, ou seja, $\operatorname{Ker}(\pi \circ \tau)=p^{i} \mathbb{Z}$ para algum inteiro $i \geq 1$. Observe que $p^{k} \in p^{i} \mathbb{Z}$ e, portanto, $i \geq k$, o que mostra que $p^{i}\langle 1\rangle \in \partial$.

Temos agora os isomorfismos de anéis

$$
\frac{\mathcal{W}(A)}{\partial} \cong \frac{\mathbb{Z}}{p^{i} \mathbb{Z}} \cong \frac{\mathcal{W}(A)}{\mathcal{P}_{\sigma, p^{i}}}
$$

onde o segundo isomorfismo é o isomorfismo encontrado no início da demonstração.

Finalmente observamos que $\mathcal{P}_{\sigma, p^{i}}=\mathcal{P}_{\sigma}+\left(p^{i}\langle 1\rangle\right)$, onde $\left(p^{i}\langle 1\rangle\right)$ denota $\circ$ ideal principal de $\mathcal{W}(A)$ gerado pelo elemento $p^{i}\langle 1\rangle$. Assim, $\mathcal{P}_{\sigma, p^{i}} \subseteq \mathcal{J}$ que, juntamente com o isomorfismo $\frac{\mathcal{W}(A)}{\mathfrak{J}} \cong \frac{\mathcal{W}(A)}{\mathcal{P}_{\sigma, p^{i}}}$, mostra que $\mathfrak{J}=\mathcal{P}_{\sigma, p^{i}}$, como queríamos.

Resumimos estes resultados em 
Teorema 4.8 Se A é um anel semilocal formalmente real, então os ideais primários de $\mathcal{W}(A)$ são:

(i) Os $\mathcal{P}_{\sigma}$, para $\sigma \in \mathcal{A} s(A)$ são os $\mathcal{P}_{\sigma}$-primários;

(ii) Os $\left(\mathcal{P}_{\sigma, p}\right)^{i}$, para $\sigma \in \mathcal{A s s}(A), p$ um número primo ímpar $e i \geq 1$, são os $\mathcal{P}_{\sigma, p}$-primários;

(iii) Os ideais contendo $2^{k}\langle 1\rangle$, para algum inteiro $k \geq 1$, são todos os $\mathfrak{I}(A)$-primários.

\subsection{Decomposição Primária em $\mathcal{W}(A)$}

Um resultado clássico de álgebra comutativa, ver por exemplo (7.13) de [01], diz que num anel noetheriano todo ideal admite uma decomposição primária, ou seja, é decomponível. Nesta seção, veremos que para anéis de Witt, vale um resultado mais forte, mais precisamente, apresentaremos condições necessárias e suficientes sobre o anel semilocal $A$ para que todo ideal de $\mathcal{W}(A)$ seja decomponível e, existem muitos tais anéis de Witt que não são noetherianos, como por exemplo, os aneis de Witt de corpos globais, ver [10]. Antes de apresentarmos tal resultado, apresentaremos um refinamento do teorema de unicidade (1.9), para o caso do anel de Witt de um anel semilocal formalmente real $A$.

Nesta seção, continuaremos assumindo que $A$ é um anel semilocal formalmente real. O próximo resultado, de verificação imediata, será frequentemente usado nas demonstrações que seguem.

Lema 4.9 Se $\sigma, \tau \in \mathcal{A s s}(A)$ são distintas, então existe $\alpha \in A^{*}$ tal que $\sigma(\langle\alpha\rangle)=1$ e $\tau(\langle\alpha\rangle)=-1$.

Dem.: Imediata. 
Seja $\mathcal{J} \subseteq \mathcal{W}(A)$ um ideal. Recordemos que o conjunto dos ideais primos associados de $\delta, A s s o c(\delta)$, é precisamente o conjunto dos ideais primos que ocorrem como radicais de ideais da forma $(\delta: b . \mathcal{W}(A)), \operatorname{com} b \in \mathcal{W}(A)$. 0 próximo resultado caracteriza quando $\mathfrak{I}(A)$ e/ou $\mathcal{P}_{\sigma}$, com $\sigma \in \mathcal{A} s s(A)$, são ideais primos associados de $\mathcal{J}$.

Proposição 4.10 Sejam $\mathfrak{I} \subseteq \mathcal{W}(A)$ um ideal e $\sigma \in \mathcal{A} s s(A)$. Temos então

(i) $\mathfrak{I}(A) \in A \operatorname{ssoc}(\mathcal{J})$ se, e somente se existe $b \in \mathcal{W}(A)-\mathcal{J}$, tal que $2^{k} b \in \mathcal{J}$, para algum inteiro positivo $k$.

(ii) Se J é decomponivel, então $\mathcal{P}_{\sigma} \in$ Assoc(d) se, e somente se $\mathcal{} \subseteq \mathcal{P}_{\sigma}$.

Dern.: Temos que $\mathfrak{I}(A) \in A \operatorname{ssoc}(\mathfrak{I})$ se, e somente se existe $b \in \mathcal{W}(A)-\mathcal{J}$ tal que $(\mathcal{J}: b . \mathcal{W}(A))$ é $\mathfrak{I}(A)$-primário. Mas, de (4.3), temos que isto ocorre se, e somente se $2^{k}\langle 1\rangle \in(\mathcal{J}: b . \mathcal{W}(A))$ para algum inteiro positivo $k$. Assim $\mathfrak{I}(A) \in A s s o c(\mathcal{J})$ se, e somente se existe $b \in \mathcal{W}(A)-\delta$ tal que $2^{k} b=2^{k}\langle 1\rangle \cdot b \in \delta$, para algum inteiro positivo $k$, o que mostra (i).

Para mostrarmos (ii), suponhamos que $\delta \subseteq \mathcal{W}(A)$ é um ideal decomponível.

Se $\mathcal{P}_{\sigma} \in A s s o c(\mathcal{J})$, então de (1.8) e da definição de $A \operatorname{ssoc}(\mathcal{J})$, temos que $\mathcal{J}$ está contido em algum ideal $\mathcal{P}_{\sigma}$-primário. Consequentemente, de (4.8), temos que $\delta \subseteq \mathcal{P}_{\sigma}$. Reciprocamente, seja $\mathcal{J} \subseteq \mathcal{P}_{\sigma}$. Suponhamos que $\mathcal{P}_{\sigma} \notin A s s o c(\mathcal{J})$. Então, usando (4.8) e o fato de $\mathcal{J}$ ser decomponível, temos que $\mathcal{\partial}$ admite uma decomposição primária da forma

$$
\delta=\left(\bigcap_{\tau \in \Gamma} \mathcal{P}_{\tau}\right) \cap\left(\bigcap_{\gamma \in \Delta}\left(\bigcap_{p \in \Delta_{\gamma}} \mathcal{P}_{\gamma, p^{i}(\gamma, p)}\right)\right) \cap Q
$$

onde $\Gamma, \Delta$ são subconjuntos finitos de $\mathcal{A}_{s s}(A), \operatorname{com} \sigma \notin \Gamma$ e, para cada $\gamma \in \Delta, \Delta_{\gamma}$ é um conjunto finito de números primos ímpares, $i(\gamma, p)$ é um inteiro $\geq 1$ para cada $\gamma \in \Delta$ e $p \in \Delta_{\gamma}$, e $Q$ é um ideal $\mathfrak{I}(A)$-primário de $\mathcal{W}(A)$ ou $Q=\mathcal{W}(A)$.

$$
\text { Seja } m_{1}=\prod_{\gamma \in \Delta}\left(\prod_{p \in \Delta_{\gamma}} p^{i(\gamma, p)}\right) \text {. Usando (4.3) se necessário, podemos afirmar que }
$$
existe um inteiro positivo $m_{2}$ tal que $2^{m_{2}}\langle 1\rangle \in Q$. Seja $m=2^{m_{2}} m_{1} \in \mathbb{Z}$. Agora, desde 
que $\sigma \notin \Gamma$, do lema anterior, temos que para cada $\tau \in \Gamma$, existe $\alpha_{r} \in A^{*}$ tal que $\sigma\left(\left\langle\alpha_{\tau}\right\rangle\right)=1$ e $\tau\left(\left\langle\alpha_{r}\right\rangle\right)=-1$. Considere

$$
b=m \prod_{\tau \in \Gamma}\left\langle 1, \alpha_{\tau}\right\rangle \in \mathcal{W}(A)
$$

Pela escolha de $m_{1}$ e $m_{2}$ feita acima, temos que $b \in Q$ e $b \in \mathcal{P}_{\gamma, p^{i}(\gamma, p)}$, para todo $\gamma \in \Delta$ e $p \in \Delta_{\gamma}$. Mais ainda, da escolha de $\alpha_{\tau} \in A^{*}$, temos que $b \in \mathcal{P}_{\tau}$, para todo $\tau \in \Gamma$, ou seja,

$$
b \in\left(\bigcap_{\tau \in \Gamma} \mathcal{P}_{\tau}\right) \cap\left(\bigcap_{\gamma \in \Delta}\left(\bigcap_{p \in \Delta_{\gamma}} \mathcal{P}_{\gamma, p^{j}(\gamma, p)}\right)\right)=\partial .
$$

Mas $\sigma(b)=2 m \neq 0$, o que contradiz a hipótese de $\partial \subseteq \mathcal{P}_{\sigma}$. Logo $\mathcal{P}_{\sigma} \in A s \operatorname{soc}(\partial)$, como queríamos.

Teorema 4.11 Seja $g \subseteq \mathcal{W}(A)$ um ideal com uma decomposição primária reduzida $Q_{1} \cap Q_{2} \cap \ldots \cap Q_{n}$.

(i) Se $r\left(Q_{i}\right)=\mathcal{P}_{\sigma}$ ou $\mathcal{P}_{\sigma, p}$, para algum $\sigma \in \mathcal{A} s s(A)$ e $p$ um primo ímpar, então $Q_{i}$ é unicamente determinado, isto é, $Q_{i}$ aparece em toda decomposição primária reduzida de g.

(ii) Todos $Q_{i}$ 's são unicamente determinados se $g \nsubseteq \mathcal{P}_{\sigma}$, para todo $\sigma \in \mathcal{A} s s(A)$ ou $2^{k} b \notin \partial$ para todo $b \notin g$ e todo inteiro positivo $k$.

Dem.: De (1.9) temos que, para mostrarmos (i), é suficiente provarmos que $r\left(Q_{i}\right)$ é um elemento minimal em Assoc(d).

Se $r\left(Q_{i}\right)=\mathcal{P}_{\sigma}$, para algum $\sigma \in \mathcal{A} s s(A)$, então de (3.15) temos que $r\left(Q_{i}\right)$ é um elemento minimal em $\mathcal{W}(A)$. Consequentemente, também o é em $A s s o c(\partial)$. Se $r\left(Q_{i}\right)=\mathcal{P}_{\sigma, p}$, para algum $\sigma \in \mathcal{A} s s(A)$ e $p$ primo ímpar, e $r\left(Q_{i}\right)$ não é minimal em Assoc(J), então de (3.15) temos que $\mathcal{P}_{\sigma}$ também é associado de $\partial$. Trocando a ordem, se necessário, de (4.8) podemos assumir que $Q_{1}=\mathcal{P}_{\sigma, p^{i}}$, para algum inteiro $i \geq 1 \mathrm{e}$ 
$Q_{2}=\mathcal{P}_{\sigma}$. Assim, $\left(Q_{1} \cap Q_{2}\right) \subseteq \mathcal{P}_{\sigma} \subseteq Q_{1}$ o que contradiz o fato de $Q_{1} \cap \ldots \cap Q_{n}$ ser uma decomposição primária reduzida de $\mathfrak{\jmath}$. Consequentemente, se $\mathcal{P}_{o, p} \in A s s o c(\mathfrak{d})$, então ele é minimal em Assoc(d), o que completa a demonstração de (i).

Suponhamos agora que $Q_{i}$ não é unicamente determinado, para algum $i=1, \ldots, n$. Então, de (1.9), temos que $A \operatorname{ssoc}(\partial)$, tem um elemento que não é minimal. Do ítem (i) acima, temos que isto ocorre somente se existe $\sigma \in \mathcal{A} s(A)$, tal que $\mathfrak{I}(A)$ e $\mathcal{P}_{\sigma}$ estão ambos em $\operatorname{Assoc}(\partial)$. Assim, $\partial \subseteq \mathcal{P}_{\sigma}$, para algum $\sigma \in \mathcal{A s s}(A)$ e, de (4.10), existe $b \in \mathcal{W}(A)-\partial$ tal que $2^{k} b \in \partial$, para algum inteiro positivo $k$, o que mostra a negação de (ii).

Para apresentarmos condiçōes necessárias e suficientes para que cada ideal do anel de Witt seja decomponível, necessitaremos de dois resultados auxiliares.

Lema 4.12 Sejam $\mathfrak{B}, \mathfrak{C}, \mathfrak{D}$ ideais de um anel $R$, com $\mathfrak{C} \subseteq \mathfrak{B}$. Então $\mathfrak{C}=\mathfrak{B} \cap(\mathfrak{C}+\mathfrak{D})$ se, e somente se $(\mathfrak{B} \cap \mathfrak{D}) \subseteq \mathfrak{C}$.

Dem.: Se $\mathfrak{C}=\mathfrak{B} \cap(\mathfrak{C}+\mathfrak{D})$ e $x \in \mathfrak{B} \cap \mathfrak{D}$, então claramente $x$ está em $\mathfrak{B} \cap(\mathfrak{C}+\mathfrak{D})=\mathfrak{C}$. Reciprocamente, se $\mathfrak{B} \cap \mathfrak{D} \subseteq \mathfrak{C}$, como $\mathfrak{C} \subseteq \mathfrak{B}$ e $\mathfrak{C} \subseteq(\mathfrak{C}+\mathfrak{D})$, temos que $\mathfrak{C} \subseteq(\mathfrak{B} \cap(\mathfrak{C}+\mathfrak{D}))$. Agora, dado $x \in(\mathfrak{B} \cap(\mathfrak{C}+\mathfrak{D}))$, podemos escrever $x=y+z$, com $y \in \mathfrak{C}$ e $z \in \mathfrak{D}$. Temos então $z=x-y$, onde $x \in \mathfrak{B}$ e $y \in \mathfrak{C} \subseteq \mathfrak{B}$, ou seja, $z \in \mathfrak{B} \cap \mathfrak{D} \subseteq \mathfrak{C}$. Assim $x=y+z \in \mathfrak{C}$, o que conclui a demonstração.

Lema 4.13 Sejam $m$ um inteiro positivo e $A$ um anel semilocal tal que $\mathcal{A s s}(A)$ tem $m+1$ elementos. Então para todo $\sigma \in \mathcal{A}$ ss $(A)$ existe uma forma bilinear $b_{\sigma} \in \operatorname{Bil}(A)$, tal que $\sigma\left(b_{\sigma}\right)=2^{m}$ e $\tau\left(b_{\sigma}\right)=0$, para toda assinatura $\tau \in \mathcal{A} s s(A) \operatorname{com} \tau \neq \sigma$.

Dem.: Dado $\sigma \in \mathcal{A s s}(A)$ fixo, para cada uma das $m$ assinaturas $\tau$ de $A$ distintas de $\sigma$, do lema (4.9) podemos encontrar $\alpha_{\tau} \in A^{*}$ tal que $\sigma\left(\left\langle\alpha_{\tau}\right\rangle\right)=1$ e $\tau\left(\left\langle\alpha_{\tau}\right\rangle\right)=-1$. 
Consideremos $b_{\sigma}=\prod_{\tau \neq \sigma}\left\langle 1, \alpha_{\tau}\right\rangle \in \mathcal{B} i l(A)$. Temos então

$\sigma\left(b_{\sigma}\right)=\prod_{\tau \neq \sigma} \sigma\left(\left\langle 1, \alpha_{\tau}\right\rangle\right)=\prod_{\tau \neq \sigma}(1+1)=2^{m}$ e $\tau\left(b_{\sigma}\right)=\prod_{\tau \neq \sigma} \tau\left(\left\langle 1, \alpha_{\tau}\right\rangle\right)=\prod_{\tau \neq \sigma}(1-1)=0$

como queríamos.

Dado um inteiro positivo $n \geq 2$ e $\sigma \in \mathcal{A s s}(A)$, denotamos por

$$
\mathcal{P}_{\sigma, n}=\{b \in \mathcal{W}(A) ; \sigma(b) \equiv 0 \bmod n\}
$$

É facil ver que se $n=p_{1}^{i_{1}} \ldots p_{k}^{i_{k}}, \operatorname{com} p_{1}, \ldots, p_{k}$ números primos distintos, então $\mathcal{P}_{\sigma, n}=\bigcap_{j=1}^{k}\left(\mathcal{P}_{\sigma, p_{j}}\right)^{i j}$. Para formalizar a notação, escrevemos $\mathcal{P}_{\sigma, 0}=\mathcal{P}_{\sigma}$ e $\mathcal{P}_{\sigma, 1}=\mathcal{W}(A)$.

Dado um anel semilocal $A$, sabemos de (3.18) e (3.22) que $A$ tem somente 2-torção. Assim, faz sentido definirmos a altura de $A$, como sendo $h(A)=2^{m}$, onde $m=\min \left\{k \in \mathbb{Z} ; k \geq 0\right.$ e $\left.2^{k} W_{t}(A)=0\right\}$ se tal número inteiro existir, caso contrário diremos que $A$ tem altura infinita e escrevemos $h(A)=\infty$. Com esta noção temos

Teorema 4.14 Todo ideal de $W(A)$ é decomponível se,e somente se $A$ tem altura finita e Ass $(A)$ é um conjunto finito.

Dem.: Suponhamos inicialmente que $A$ é um anel semilocal com $h(A) \leq 2^{k}$ e $\mathcal{A} s s(A)=\left\{\sigma_{0}, \sigma_{1}, \ldots, \sigma_{m}\right\}$.

Seja $\mathfrak{J} \subseteq \mathcal{W}(A)$ um ideal próprio. Queremos mostrar que $\mathcal{J}$ é decomponivel. Para tanto, para cada $i=0,1, \ldots, m$, considere $\mathfrak{B}_{\mathfrak{i}}=\left\{\sigma_{i}(b) ; b \in \mathfrak{J}\right\} \subseteq \mathbb{Z}$. É fácil ver que $\mathfrak{B}_{i}$ é um ideal de $\mathbb{Z}$, gerado por digamos $2^{r_{i}} n_{i}$, com $r_{i} \geq 0$ e $n_{i}$ um inteiro impar ou $n_{i}=0$. Sejam $b_{i}^{\prime} \in J_{j} i=0,1, \ldots, m$, tais que $\sigma_{i}\left(b_{i}^{\prime}\right)=2^{r_{i}} n_{i}$. Para $r=\max \left\{r_{0}, r_{1}, \ldots, r_{m}\right\}$ e $b_{i}=2^{r-r_{i}} b_{i}^{\prime}, i=0,1, \ldots, m$, vamos mostrar que

$$
J=\left(\bigcap_{i=0}^{m} \mathcal{P}_{\sigma_{i}, n_{i}}\right) \cap\left(\mathcal{J}+2^{k+m+r} \mathcal{W}(A)\right) \text {. }
$$


Se $b \in \mathcal{J}$, então para cada $i=0,1, \ldots, m$, temos que $\sigma_{i}(b) \in \mathfrak{B}_{\mathfrak{i}}$, o que implica que $\sigma_{i}(b)$ é um múltiplo de $2^{r_{i}} n_{i}$ e portanto, $\sigma_{i}(b) \equiv 0 \bmod n_{i}$. Assim $b \in \mathcal{P}_{\sigma_{i}, n_{i}}$, o que mostra que $\mathfrak{J} \subseteq\left(\bigcap_{i=0}^{m} \mathcal{P}_{\sigma_{i}, n_{i}}\right)$. Agora, considerando $\mathfrak{B}=\bigcap_{i=0}^{m} \mathcal{P}_{\sigma_{i}, n_{i}}, \mathfrak{C}=\mathfrak{J}$ e $\mathfrak{D}=$ $2^{k+m+r} \mathcal{W}(A)$ no lema (4.12), temos que é suficiente mostrar que

$$
\left(\bigcap_{i=0}^{m} \mathcal{P}_{\sigma_{i}, n_{i}}\right) \cap\left(2^{k+m+r} \mathcal{W}(A)\right) \subseteq \mathfrak{J}
$$

Seja $b \in\left(\bigcap_{i=0}^{m} \mathcal{P}_{\sigma_{i}, n_{i}}\right) \cap\left(2^{k+m+r} \mathcal{W}(A)\right)$. Escrevendo $b=2^{k+m+r} b_{0}$, com $b_{0} \in \mathcal{W}(A)$, temos que $\sigma_{i}(b)=2^{k+m+r} \sigma_{i}\left(b_{0}\right) \equiv 0 \bmod n_{i}$, para cada $i=0,1, \ldots, m$. Desde que $n_{i}$ é ímpar ou zero, temos que $\sigma_{i}\left(b_{0}\right)=s_{i} n_{i}$, para algum inteiro $s_{i}$. Do lema anterior temos que, para cada $i=0,1, \ldots, m$, existe uma forma bilinear $q_{i}$ tal que $\sigma_{i}\left(q_{i}\right)=2^{m}$ e $\sigma_{j}\left(q_{i}\right)=0$ se $j \neq i$.

Considere $b^{\prime}=\prod_{i=0}^{m} s_{i} q_{i} \cdot b_{i}$. Observe que $b^{\prime} \in \mathcal{J}$, pois cada $b_{i} \in \mathcal{J}$. Mais ainda, da construção dos $b_{i}$ 's, temos que para cada $i=0,1, \ldots, m$,

$$
\sigma_{i}\left(b^{\prime}\right)=\sum_{j=0}^{m} s_{j} \sigma_{i}\left(q_{j}\right) \sigma_{i}\left(b_{j}\right)=s_{i} 2^{m} \sigma_{i}\left(2^{r-r_{i}} b_{i}^{\prime}\right)=2^{m+r} s_{i} n_{i} .
$$

Logo $2^{m+r} b_{0}-b^{\prime} \in \mathcal{W}(A)$ é tal que $\sigma_{i}\left(2^{m+r} b_{0}-b^{\prime}\right)=0$, para todo $i=0,1, \ldots, m$, o que implica que $2^{m+r} b_{0}-b^{\prime} \in$ mathscr $W_{t}(A)$ pelo Princípio Local-Global de Pfister. Assim, desde que $h(A) \leq 2^{k}$, obtemos $b-2^{k} b^{\prime}=2^{k}\left(2^{m+r} b_{0}-b^{\prime}\right)=0$, ou seja, $b=2^{k} b^{\prime} \in \mathcal{J}$. Consequentemente, da definição de $\mathcal{P}_{\sigma_{i}, n_{i}}$ e de (4.6) e (4.8) temos que

$$
\left(\bigcap_{i=0}^{m} P_{\sigma_{i}, n_{i}}\right) \cap\left(\mathfrak{J}+2^{k+m+r} \mathcal{W}(A)\right)
$$

é uma decomposição primária de $\mathfrak{J}$.

Para a recíproca, consideremos que $A$ é um anel semilocal tal que todo ideal de $\mathcal{W}(A)$ é decomponível. Queremos mostrar que $h(A)<\infty$ e $\mathcal{A s s}(A)$ é um conjunto finito. 
Suponhamos que $A$ admita infinitas assinaturas. Considere o ideal de torção $\mathcal{W}_{t}(A)$ que por hipótese é decomponível. Logo $\mathcal{W}_{t}(A)$ se escreve como uma interseç̧ão finita de ideais primários de $\mathcal{W}(A)$. Do Princípio Local-Global de Pfister, temos que $\mathcal{W}_{t}(A) \subseteq \mathcal{P}_{\sigma}$, para toda $\sigma \in \mathcal{A} s(A)$. Como, $\mathcal{P}_{\sigma} \subseteq \mathcal{P}_{\sigma, p}$, podemos assumir que nenhum ideal primário da forma $\mathcal{P}_{\sigma, p^{i}}$, onde $\sigma \in \mathcal{A} s s(A), p$ primo ímpar e $i \geq 1$, ocorre na decomposição de $\mathcal{W}_{t}(A)$. Assim, usando (4.8), temos que existem $\sigma_{1}, \ldots, \sigma_{l} \in \mathcal{A} s s(A)$ tais que

$$
\mathcal{W}_{t}(A)=\left(\bigcap_{i=1}^{l} \mathcal{P}_{\sigma_{i}}\right) \cap Q
$$

onde $Q$ é um ideal $\mathfrak{I}(A)$-primário, ou $Q=\mathcal{W}(A)$. De (4.3) temos que existe um inteiro positivo $r$ tal que $2^{r}\langle 1\rangle \in Q$ e, como $\mathcal{A s s}(A)$ é um conjunto infinito, existe $\gamma \in \mathcal{A s s}(A)$, com $\gamma \neq \sigma_{i}$, para todo $i=1, \ldots, l$. Para cada $i=1, \ldots, l$, considere $\alpha_{i} \in A^{*}$ tal que $\gamma\left(\left\langle\alpha_{i}\right\rangle\right)=1$ e $\sigma_{i}\left(\left\langle\alpha_{i}\right\rangle\right)=-1$, que existem pelo lema (4.9). Tomando $b=2^{r}\left\langle 1, \alpha_{1}\right\rangle \otimes\left(1, \alpha_{2}\right\rangle \otimes \ldots \otimes\left\langle 1, \alpha_{l}\right\rangle$, temos que $b \in\left(\bigcap_{i=1}^{l} \mathcal{P}_{\sigma_{i}}\right) \cap Q=\mathcal{W}_{t}(A)$. Mas $\gamma(b)=2^{r+l} \neq 0$, o que contradiz o Princípio Local-Global de Pfister. Consequentemente, se todo ideal de $\mathcal{W}(A)$ é decomponível, então $\mathcal{A} s s(A)$ é um conjunto finito.

Finalmente, suponhamos que $\mathcal{A} s s(A)$ é um conjunto finito e $h(A)=\infty$. Como $h(A)=\infty$, temos que $\mathcal{W}_{t}(A) \neq 0$. Seja $b \in \mathcal{W}_{t}(A)$, com $b \neq 0$. 0 ideal principal $\mathfrak{B}=b \cdot \mathcal{W}(A)$ está contido em $\mathcal{P}_{\sigma}$, para toda $\sigma \in \mathcal{A} s s(A)$. Assim, de (4.8) podemos assumir que $\mathfrak{B}$ tem uma decomposição primária da forma

$$
\mathfrak{B}=\left(\bigcap_{\sigma \in \mathcal{A} s s(A)} \mathcal{P}_{\sigma}\right) \cap Q,
$$

onde $Q$ é $\mathfrak{I}(A)$-primário ou $Q=\mathcal{W}(A)$. Agora, como $b$ é um elemento de torção de $\mathcal{W}(A)$, de (3.22) temos que existe um inteiro $m \geq 1$ tal que $2^{m} b=0$. Mais ainda, de (4.3) temos que existe um inteiro positivo $k$ tal que $2^{k}\langle 1\rangle \in Q$. Logo o ideal $Q$ contém os ideais $\mathfrak{B}$ e $2^{k} \mathcal{W}(A)$ e, sem perda de generalidade, podemos assumir $k \geq m$. 
Temos então

$$
\mathfrak{B} \subseteq\left(\bigcap_{\sigma \in \mathcal{A} s s(A)} \mathcal{P}_{\sigma}\right) \cap\left(\mathfrak{B}+\left(2^{k} \mathcal{W}(A)\right)\right) \subseteq\left(\bigcap_{\sigma \in \mathcal{A} s s(A)} \mathcal{P}_{\sigma}\right) \cap Q=\mathfrak{B},
$$

ou seja,

$$
\mathfrak{B}=\left(\bigcap_{\sigma \in \mathcal{A} s(A)} \mathcal{P}_{\sigma}\right) \cap\left(\mathfrak{B}+2^{k} \mathcal{W}(A)\right)=\mathcal{W}_{t}(A) \cap\left(\mathfrak{B}+2^{k} \mathcal{W}(A)\right),
$$

onde a última igualdade segue do Princípio Local-Global de Pfister. Em particular, temos $2^{k} \mathcal{W}_{t}(A)=\mathcal{W}_{t}(A) \cap\left(2^{k} \mathcal{W}(A)\right) \subseteq \mathfrak{B}$

Agora, desde que $h(A)=\infty$, temos que existe $b_{0} \in W_{t}(A)$ tal que $2^{2 k} b_{0} \neq 0$ em $\mathcal{W}(A)$. Mas $2^{k} b_{0} \in 2^{k} \mathcal{W}_{t}(A) \subseteq \mathfrak{B}=b . \mathcal{W}(A)$. Então $2^{k} b_{0}=b . b_{1}$, para algum $b_{1} \in \mathcal{W}(A)$, o que implica que $2^{2 k} b_{0}=2^{k} b . b_{1}=0$, o que é uma contradição. Portanto, $h(A)<\infty$ o que completa a demonstração do teorema.

\subsection{Ideais contendo uma forma de dimensão ímpar}

Nesta seção assumiremos que $A$ é um anel semilocal formalmente real, com $h(A)<\infty$ e com um conjunto finito de assinaturas. Sob tais condições apresentaremos alguns resultados sobre ideais de $\mathcal{W}(A)$ que contém uma forma de dimensão ímpar, ou seja, ideais que não estão contidos em $\mathfrak{I}(A)$. Encontraremos também condições equivalentes para que formas de dimensão ímpar tenham fatoração única como produto de irredutíveis.

Seja $\partial \subseteq \mathcal{W}(A)$ um ideal contendo uma forma de dimensão ímpar. Desde que, de (3.15), $\mathcal{P}_{\sigma} \subseteq \mathfrak{I}(A)$, para todo $\sigma \in \mathcal{A s s}(A)$ e $\mathcal{J} \nsubseteq \mathfrak{I}(A)$, temos que $\mathcal{J} \nsubseteq \mathcal{P}_{\sigma}$, para todo $\sigma \in \mathcal{A s s}(A)$. Usando a caracterização dos ideais primários de $\mathcal{W}(A)$, apresentada em (4.8), temos que $\partial$ pode ser escrito como uma intersecção finita de ideais da forma $\mathcal{P}_{\sigma, p^{i}}, \operatorname{com} \sigma \in \mathcal{A s s}(A), p$ primos ímpares e $i \geq 1$. Além disso, de (4.11), temos que esta decomposição primária reduzida é unicamente determinada. 
Proposição 4.15 $S e \mathfrak{J} \subseteq \mathcal{W}(A)$ é um ideal contendo uma forma de dimensão ímpar então, para todo $b \in \mathcal{W}(A)-\mathcal{J}$, temos que $2^{k} b \notin \mathcal{J}$, para todo inteiro $k \geq 0$.

Dem.: Desde que todo ideal primário está contido em seu radical e $\mathfrak{J} \nsubseteq \mathfrak{I}(A)$, temos que nenhum ideal $\mathfrak{I}(A)$-primário de $\mathcal{W}(A)$ está contido em $\mathcal{J}$. Assim, da definição de Assoc(J), temos que $\mathfrak{I}(A) \notin$ Assoc(J). Agora, a demonstração segue de (4.10).

Proposição 4.16 Se $\mathfrak{\partial}$ é um ideal de $\mathcal{W}(A)$ contendo uma forma de dimensão impar, então $\mathcal{W}_{t}(A) \subseteq$ J.

Dem.: Pelo Princípio Local-Global de Pfister, obtemos que $\mathcal{W}_{t}(A) \subseteq \mathcal{P}_{\sigma, p^{i}}$, para todo $\sigma \in \mathcal{A} s s(A), p$ primo ímpar e $i \geq 1$. Logo, o resultado segue da observação feita no início desta seção.

Proposição 4.17 $S e b_{1}$ e $b_{2}$ são formas bilineares de dimensão impar tais que $b_{1}-b_{2} \in \mathcal{W}_{t}(A)$, então os ideais principais $b_{1} . \mathcal{W}(A)$ e $b_{2} . \mathcal{W}(A)$ são iguais.

Dem.: Como $b_{1}-b_{2} \in \mathcal{W}_{t}(A)$, pelo Principio Local-Global de Pfister, temos que $\sigma\left(b_{1}\right)=\sigma\left(b_{2}\right)$, para todo $\sigma \in \mathcal{A} s s(A)$. Assim, para todo primo ímpar $p, b_{1} \in \mathcal{P}_{\sigma_{\mathrm{t}} p^{i}}$ se, e somente se $b_{2} \in \mathcal{P}_{\sigma, p^{i}}$, onde $\sigma \in \mathcal{A} s s(A)$ e $i \geq 1$. Consequentemente, os ideais primários que aparecem nas decomposições primárias reduzidas dos ideais $b_{1} \cdot \mathcal{W}(A)$ e $b_{2} \cdot \mathcal{W}(A)$ são os mesmos e, portanto, $b_{1} \cdot \mathcal{W}(A)=b_{2} \cdot \mathcal{W}(A)$.

Dados $\alpha_{1}, \alpha_{2}, \ldots, \alpha_{n} \in A^{*}$, dizemos que a forma bilinear não singular

$$
b=\left\langle 1, \alpha_{1}\right\rangle \otimes\left\langle 1, \alpha_{2}\right\rangle \otimes \ldots \otimes\left\langle 1, \alpha_{n}\right\rangle
$$

é uma $n$-forma de Pfister e, denotamos por $b=\left\langle\left\langle\alpha_{1}, \ldots, \alpha_{n}\right\rangle\right\rangle$. Para $\sigma \in \mathcal{A} s s(A)$, temos que $\sigma((\alpha\rangle)= \pm 1$, para cada $\alpha \in A^{*}$. Assim se $b$ é uma $n$-forma de Pfister, 
então $\sigma(b)=0$ ou $\sigma(b)=2^{n}=\operatorname{dim}(b)$, para cada $\sigma \in \mathcal{A} s s(A)$. Mais ainda, se $b$ é uma $n$-forma de Pfister, então $b=\langle 1\rangle \perp b^{\prime}$, para algum $b^{\prime} \in \mathcal{B} i l(A)$. Outra notação que usaremos no próximo resultado é que dado $b \in \mathcal{B} i l(A)$, o conjunto dos elementos de $A^{*}$ representados por $b$ são denotados por $\mathcal{D}(b)$, ou seja, se $M$ é um $A$-módulo livre de dimensão finita e $(M, b)$ é um espaço bilinear sobre $A$, então

$$
\mathcal{D}(b)=\left\{\alpha \in A^{*} ; b(x, x)=\alpha, \text { para algum } x \in M\right\} .
$$

Com estas notações temos a seguinte conseqüència da proposição anterior

Corolário 4.18 Sejam $b_{1}=\langle 1\rangle \perp b_{1}^{\prime}$ e $b_{2}=\langle 1\rangle \perp b_{2}^{\prime}$ duas $n$-formas de Pfister sobre A. Se $\mathcal{D}\left(b_{1}^{\prime}\right)=\mathcal{D}\left(b_{2}^{\prime}\right)$, entâo existe uma unidade $b \in \mathcal{W}(A)$ tal que $b_{1}^{\prime}=b . b_{2}^{\prime}$.

Dem.: Sejam $\alpha_{i}, \beta_{i} \in A^{*}, i=1, \ldots, n$, tais que $b_{1}=\left\langle\left\langle\alpha_{1}, \ldots, \alpha_{n}\right\rangle\right\rangle$ e $b_{2}=$ $\left\langle\left\langle\beta_{1}, \ldots, \beta_{n}\right\rangle\right\rangle$. Dado $\sigma \in \mathcal{A} s s(A)$, afirmamos que $\mathcal{D}\left(b_{1}^{\prime}\right)=\mathcal{D}\left(b_{2}^{\prime}\right)$, implica que $\sigma\left(b_{1}\right)=$ $2^{n}$ se, e somente se $\sigma\left(b_{2}\right)=2^{n}$. De fato, se $\sigma\left(b_{1}\right)=2^{n}$ e $\sigma\left(b_{2}\right)=0$, então temos que $\sigma\left(\left\langle\alpha_{i}\right\rangle\right)=1$, para todo $i=1, \ldots, n$ e existe $j \in\{1, \ldots, n\}$ tal que $\sigma\left(\left\langle\beta_{j}\right\rangle\right)=-1$. Mas $\beta_{j} \in \mathcal{D}\left(b_{2}^{\prime}\right)=\mathcal{D}\left(b_{1}^{\prime}\right)$. Assim, de (2.8) temos que existe $b_{3} \in \mathcal{B} i l(A)$ tal que $b_{1}^{\prime} \simeq\left\langle\beta_{j}\right\rangle \perp b_{3}$. Como $b_{1}=\langle 1\rangle \perp b_{1}^{\prime}$ e $\sigma\left(b_{1}\right)=2^{n}=\operatorname{dim}\left(b_{1}\right)$, temos que $\sigma\left(b_{1}^{\prime}\right)=$ $2^{n}-1=\operatorname{dim}\left(b_{1}^{\prime}\right)$. Consequentemente, $\operatorname{dim}\left(b_{1}^{\prime}\right)=\sigma\left(b_{1}^{\prime}\right)=\sigma\left(\left\langle\beta_{j}\right\rangle \perp b_{3}\right)=-1+\sigma\left(b_{3}\right) \leq$ $\operatorname{dim}\left(b_{3}\right)-1=\left(\operatorname{dim}\left(b_{1}^{\prime}\right)-1\right)-1<\operatorname{dim}\left(b_{1}^{\prime}\right)$, o que é uma contradição.

Usando o fato que $\sigma\left(b_{i}\right)=1+\sigma\left(b_{i}^{\prime}\right)$, para $i=1,2$ e $\sigma \in \mathcal{A} s s(A)$, e a afirmação acima, temos que, para cada $\sigma \in \mathcal{A} s s(A), \sigma\left(b_{1}^{\prime}\right)=\sigma\left(b_{2}^{\prime}\right)$, o que mostra que $b_{1}^{\prime}-b_{2}^{\prime}$ está em $\mathcal{W}_{t}(A)$ pelo Princípio Local-Global de Pfister. Como $\operatorname{dim}\left(b_{i}^{\prime}\right)=2^{\text {n }}-1$ é impar, para $i=1,2$ temos da proposição anterior que $b_{1}^{\prime} \cdot \mathcal{W}(A)=b_{2}^{\prime} \cdot \mathcal{W}(A)$, ou seja, existe um elemento inversível $b \in \mathcal{W}(A)$ tal que $b_{1}^{\prime}=b . b_{2}^{\prime}$, como queríamos.

Observação 4.19 Ainda é um problema em aberto se as hipóteses de (4.18), de fato implicam que $b_{1} \simeq b_{2}$. 
O próximo teorema mostra condições equivalentes para que os ideais de $\mathcal{W}(A)$ que não estão contidos em $\mathfrak{I}(A)$ sejam ideais principais.

Teorema 4.20 As seguintes afirmações são equivalentes:

(i) $\mathcal{P}_{\sigma, 3}$ é um ideal principal, para todo $\sigma \in \mathcal{A s s}(A)$;

(ii) Para cada $\sigma \in \mathcal{A} s s(A)$, existe uma forma bilinear $b$ sobre $A$, tal que $\sigma(b)=3 e$ $\tau(b)=-1$, para todo $\tau \in \mathcal{A s s}(A), \operatorname{com} \tau \neq \sigma ;$

(iii) Para cada $\sigma \in \mathcal{A s s}(A)$, existe uma forma bilinear b sobre $A$, tal que $\sigma(b)=4 e$ $\tau(b)=0$, para todo $\tau \in \mathcal{A} s s(A), \operatorname{com} \tau \neq \sigma ;$

(iv) Todo ideal de $\mathcal{W}(A)$ contendo uma forma de dimensäo ímpar é um ideal principal.

Dem.: É evidente que (iv) $\Longrightarrow$ (i). Mostremos então as implicações (i) $\Longrightarrow$ (ii) $\Longrightarrow$ (iii) $\Longrightarrow$ (iv).

(i) $\Longrightarrow$ (ii). Dado $\sigma \in \mathcal{A} s s(A)$, temos que $\sigma\left(\mathcal{P}_{\sigma, 3}\right)=3 \mathbb{Z}$. Logo existe um gerador $b_{1}$ do ideal principal $\mathcal{P}_{\sigma, 3}$ tal que $\sigma\left(b_{1}\right)=3$. De (3.15) temos que $\mathcal{P}_{\sigma, 3}$ contém um único ideal primo minimal, que é $\mathcal{P}_{\sigma}$. Assim, se $\tau \in \mathcal{A} s s(A)$ é tal que $\tau \neq \sigma$, então $\mathcal{P}_{\tau} \nsubseteq \mathcal{P}_{\sigma, 3}$ e, como $\mathcal{P}_{\sigma, 3}$ é um ideal maximal de $\mathcal{W}(A)$, temos que $\mathcal{P}_{\sigma, 3}+\mathcal{P}_{\tau}=\mathcal{W}(A)$. Usando que $\mathcal{P}_{\sigma, 3}=b_{1} \cdot \mathcal{W}(A)$ e que $\mathcal{P}_{\tau}=\operatorname{Ker}(\tau)$, temos que $\mathbb{Z}=\tau(\mathcal{W}(A))=\tau\left(\mathcal{P}_{\sigma, 3}\right)=$ $\tau\left(b_{1} . \mathcal{W}(A)\right)=\tau\left(b_{1}\right) \mathbb{Z}$, o que mostra que $\tau\left(b_{1}\right)= \pm 1$, para todo $\tau \in \mathcal{A s s}(A)$ com $\tau \neq \sigma$.

Agora, do fato que $\sigma\left(b_{1}\right)=3$, obtemos que $\operatorname{dim}\left(b_{1}\right)$ é ímpar. Usando (3.3) e o fato que $\tau\left(b_{1}\right)= \pm 1$ e $\sigma\left(b_{1}\right)=3$, para cada $\tau \in \mathcal{A s s}(A)$, com $\tau \neq \sigma$, temos que $b_{1}=\left\langle\alpha_{1}, \ldots, \alpha_{2 n+1}\right\rangle$ em $\mathcal{W}(A)$, com $\alpha_{i} \in A^{*}$ tais que

$$
\tau\left(\left\langle\alpha_{i}\right\rangle\right)=\left\{\begin{aligned}
1 & \text { se } 1 \leq i \leq n \\
-1 & \text { se } n+1 \leq i \leq 2 n \\
\tau(b) & \text { se } i=2 n+1
\end{aligned}\right.
$$


e,

$$
\sigma\left(\left\langle\alpha_{i}\right\rangle\right)=\left\{\begin{aligned}
-1 & \text { se } 1 \leq i \leq n-1 \\
1 & \text { se } n \leq i \leq 2 n+1
\end{aligned}\right.
$$

Seja $e=(-1)^{n} \alpha_{1} \ldots \alpha_{2 n+1} \in A^{*}$. Então,

$$
\tau(\langle e\rangle)=(-1)^{n} \prod_{i=1}^{2 n+1} \tau\left(\left\langle\alpha_{i}\right\rangle\right)=(-1)^{n}(-1)^{n} \tau\left(\left\langle\alpha_{2 n+1}\right\rangle\right)=\tau\left(b_{1}\right)
$$

para cada $\tau \in \mathcal{A s s}(A), \operatorname{com} \tau \neq \sigma \mathrm{e}$,

$$
\sigma(\langle e))=(-1)^{n} \prod_{i=1}^{2 n+1} \sigma\left(\left\langle\alpha_{i}\right\rangle\right)=(-1)^{n}(-1)^{n-1}=-1 .
$$

Assim, $b=\langle-e) \otimes\left\langle\alpha_{1}, \alpha_{2}, \ldots, \alpha_{2 n+1}\right\rangle$ é uma forma bilinear não singular sobre $A$, tal que

$$
\sigma(b)=\sigma(\langle-e)) \sum_{i=1}^{2 n+1} \sigma\left(\left\langle\alpha_{i}\right\rangle\right)=-\sigma(\langle e\rangle) 3=3
$$

e, para cada $\tau \in \mathcal{A s s}(A), \operatorname{com} \tau \neq \sigma$,

$$
\tau(b)=\tau(\langle-e)) \sum_{i=1}^{2 n+1} \tau\left(\left\langle\alpha_{i}\right)\right)=-\tau\left(b_{1}\right)^{2}=-1,
$$

pois $\tau\left(b_{1}\right)= \pm 1$, o que mostra (ii).

(ii) $\Longrightarrow$ (iii). Se $b$ é uma forma bilinear satisfazendo a condição (ii), então $b \perp\langle 1$ ) satisfaz (iii).

(iii) $\Longrightarrow$ (iv). Para $\sigma \in \mathcal{A s s}(A)$, seja $b_{\sigma}$ a forma bilinear satisfazendo (iii), ou seja, $\sigma\left(b_{\sigma}\right)=4$ e $\tau\left(b_{\sigma}\right)=0$, para todo $\tau \in \mathcal{A s s}(A), \operatorname{com} \tau \neq \sigma$. Multiplicando $b_{\sigma}$ por algum $\alpha \in \mathcal{D}\left(b_{\sigma}\right)$, com $\sigma(\langle\alpha\rangle)=1$, se necessário, podemos assumir que $1 \in \mathcal{D}\left(b_{\sigma}\right)$. Então, de (2.8) temos que $b_{\sigma} \simeq\langle 1) \perp q_{\sigma}$, para algum $q_{\sigma} \in \mathcal{B} i l(A)$. Observe que, neste caso, para $\tau \in \mathcal{A s s}(A)$,

$$
\tau\left(q_{\sigma}\right)=\left\{\begin{array}{rr}
3 & \text { se } \tau=\sigma \\
-1 & \text { se } \tau \neq \sigma
\end{array}\right.
$$


Para mostrarmos a conclusão apresentada em (iv), usando a observação feita no início desta seção, é suficiente mostrarmos que os ideais $\mathcal{J}=\bigcap_{i \in \Gamma} \mathcal{P}_{\sigma, p^{i}}$ são principais, onde $\Gamma$ é um conjunto finito de pares $\left(\sigma, p^{i}\right), \operatorname{com} \sigma \in \mathcal{A s s}(A), p$ primos ímpares e $i \geq 1$ inteiro.

Para cada $\sigma \in \mathcal{A s s}(A)$, seja $n_{\sigma}=\prod_{\left(\sigma, p^{i}\right) \in \Gamma} p^{i}, \operatorname{com} n_{\sigma}=1$ se este produto for vazio e $\mathcal{P}_{\sigma, 1}=\mathcal{W}(A)$. Com esta notação, desde que $\mathcal{A} s s(A)$, é um conjunto finito, temos que $\mathfrak{J}=\bigcap_{\sigma \in \mathcal{A} s s(A)} \mathcal{P}_{\sigma, n_{\sigma}}$. Agora, o resultado segue da seguinte afirmação

Afirmação - Existe uma forma bilinear $b \in \operatorname{Bil}(A)$, tal que $|\sigma(b)|=n_{\sigma}$, para todo $\sigma \in \mathcal{A s s}(A)$.

De fato, se tal forma bilinear $b$ existe, então $b . \mathcal{W}(A) \subseteq \mathcal{P}_{\sigma, n_{\sigma}}$, para todo $\sigma \in \mathcal{A} s s(A)$, o que implica que $b . \mathcal{W}(A) \subseteq \bigcap_{\sigma \in \mathcal{A} s s(A)} \mathcal{P}_{\sigma, n_{\sigma}}=\mathcal{J}$. Observe que para cada $\sigma \in \mathcal{A} s s(A), n_{\sigma}$ é um número inteiro ímpar e, $\sigma(b)=n_{\sigma}$ implica que $b$ é uma forma de dimensão ímpar. Portanto, os únicos ideais primários contendo $b$ são os ideais $\mathcal{P}_{\sigma, p^{i}}$, com $\left(\sigma, p^{i}\right) \in \Gamma$ e ideais contendo estes. Desde que a decomposição primária reduzida de $b . \mathcal{W}(A)$ é uma intersecção finita de ideais primários contendo $b$, temos que $\mathcal{\partial} \subseteq b . \mathcal{W}(A)$, ou seja, $\mathcal{J}=b . \mathcal{W}(A)$, o que mostra (iv).

Finalmente, mostremos a afirmação. Se $n_{\sigma}=1$, para todo $\sigma \in \mathcal{A} s s(A)$, então $b=\langle 1\rangle$ satisfaz a afirmação. Podemos então assumir que $n_{\sigma} \neq 1$, para pelo menos um $\sigma \in \mathcal{A} s s(A)$. Como $n_{\sigma}$ é um inteiro ímpar, para cada $\sigma \in \mathcal{A} s s(A)$, temos que existe pelo menos um $\sigma \in \mathcal{A} s s(A)$ tal que $n_{\sigma} \geq 3$.

Desde que $\mathcal{A} s s(A)$ é um conjunto finito, temos que $n=\sum_{\sigma \in \mathcal{A} s s(A)} n_{\sigma} \geq r+2$, onde $r$ é o número de elementos de $\mathcal{A s s}(A)$. Mostraremos agora a afirmação por indução sobre $n$.

Se $n=r+2$, então existe exatamente um $\sigma \in \mathcal{A} s s(A)$ tal que $n_{\sigma}=3$ e $n_{\tau}=1$, para todo $\tau \in \mathcal{A s s}(A)$ com $\tau \neq \sigma$. Neste caso, $b=q_{\sigma}$ satisfaz o requerido.

Se $n>r+2$, consideremos dois casos separadamente: 
Caso 1 - Existe $\sigma \in \mathcal{A s s}(A)$, tal que $n_{\sigma} \geq 5$.

Considere o ideal de $\mathcal{W}(A), \partial_{0}=\mathcal{P}_{\sigma, n_{\sigma}-4} \cap\left(\bigcap_{\tau \neq \sigma} \mathcal{P}_{\tau, n_{\tau}}\right)$. Neste caso, $n_{0}=$ $\left(n_{\sigma}-4\right)+\sum_{\tau \neq \sigma} n_{\tau}<n$ e, por hipótese de indução, existe $b_{0} \in \mathcal{B i l}(A)$ tal que

$$
\left|\tau\left(b_{0}\right)\right|= \begin{cases}n_{\sigma}-4 & \text { se } \tau=\sigma \\ n_{\tau} & \text { se } \tau \neq \sigma\end{cases}
$$

Tomando $e= \pm 1$, de acordo com o sinal de $\sigma\left(b_{0}\right)= \pm\left(n_{\sigma}-4\right)$, temos que $b=$ $b_{0} \perp\langle e\rangle \otimes b_{\sigma}$ satisfaz a afirmação pois

$$
\begin{aligned}
& |\sigma(b)|=\left|\sigma\left(b_{0}\right)+\sigma\left(\langle e\rangle \otimes b_{\sigma}\right)\right|=\left(n_{\sigma}-4\right)+4=n_{\sigma} \\
& |\tau(b)|=\left|\tau\left(b_{0}\right)+\tau\left(\langle e\rangle \otimes b_{\sigma}\right)\right|=\left|\tau\left(b_{0}\right)\right|=n_{\tau},
\end{aligned}
$$

para todo $\tau \neq \sigma$.

Caso 2 - Para todo $\sigma \in \mathcal{A s s}(A), n_{\sigma} \leq 3$.

Desde que $n>r+2$, temos que pelo menos dois $n_{\sigma}$ 's são iguais a 3. Digamos que $\sigma, \gamma \in \mathcal{A} s s(A)$ são tais que $n_{\sigma}=n_{\gamma}=3$. Seja

$$
\partial_{0}=\mathcal{P}_{\sigma, 1} \cap \mathcal{P}_{\gamma, 1} \cap\left(\bigcap_{\tau \neq \sigma, \gamma} P_{\tau, n_{+}}\right) \subseteq \mathcal{W}(A)
$$

Para este ideal $\partial_{0}$, temos $n_{0}=1+1+\sum_{\tau \neq \sigma, \gamma} n_{\tau}<n$. Então, por hipótese de indução, existe $b_{0} \in \mathcal{B i l}(A)$ tal que

$$
\left|\tau\left(b_{0}\right)\right|= \begin{cases}1 & \text { se } \tau=\sigma \text { ou } \gamma \\ n_{\tau} & \text { se } \tau \neq \sigma, \gamma\end{cases}
$$

Para cada $\tau \in \mathcal{A s s}(A)$, seja $e_{\tau}=\frac{\tau\left(b_{0}\right)}{\left|\tau\left(b_{0}\right)\right|}= \pm 1$, de acordo com o sinal de $\tau\left(b_{0}\right)$. Sem perda de generalidade, podemos assumir que $e_{\sigma}=1$, pois caso contrário, $b_{0}^{\prime}=\langle-1\rangle \otimes b_{0} \in \mathcal{B i l}(A)$ é tal que $\left|\tau\left(b_{0}^{\prime}\right)\right|=\left|\tau\left(b_{0}\right)\right|$, para todo $\tau \in \mathcal{A s s}(A)$, e $e_{\sigma}=\frac{\sigma\left(b_{0}^{\prime}\right)}{\sigma\left(b_{0}^{\prime}\right)}=1$. Mais ainda, podemos também assumir que $e_{\gamma}=1$, pois se $e_{\sigma}=$ 
1 e $e_{\gamma}=-1$, escolhemos $\alpha \in A$ tal que $\sigma(\langle\alpha\rangle)=1$ e $\gamma(\langle\alpha\rangle)=-1$, que existe por (4.9). Neste caso, $b_{0}^{\prime}=\langle\alpha\rangle \otimes b_{0} \in \mathcal{B} i l(A)$ é tal que $\left|\tau\left(b_{0}^{\prime}\right)\right|=\left|\tau\left(b_{0}\right)\right|$, para todo $\tau \in \mathcal{A} s s(A)$ e $e_{\sigma}=e_{\gamma}=1$.

Para cada $\tau \in \mathcal{A} s s(A), \operatorname{com} \tau \neq \sigma, \gamma$, podemos escrever $n_{\tau}=2 m_{\tau}^{\prime}+1$, pois cada $n_{\tau}$ é ímpar. Considerando $m_{\tau}=m_{\tau}^{\prime}$ se $e_{\tau}=1$ e $m_{\tau}=-\left(m_{\tau}^{\prime}+1\right)$ se $e_{\tau}=-1$, temos que

$$
b=b_{0} \perp q_{\sigma} \perp q_{\gamma} \perp\left(\underset{\tau \neq \sigma, \gamma}{\perp}\left(-m_{\tau}\right) b_{\tau}\right)
$$

satisfaz a afirmação. De fato:

$$
\left\{\begin{array}{l}
\sigma(b)=\sigma\left(b_{0}\right)+\sigma\left(q_{\sigma}\right)+\sigma\left(q_{\gamma}\right)-\sum_{\tau \neq \sigma, \gamma} m_{\tau} \sigma\left(b_{\tau}\right)=1+3-1=3=n_{\sigma} \\
\gamma(b)=\gamma\left(b_{0}\right)+\gamma\left(q_{\sigma}\right)+\gamma\left(b_{\gamma}\right)-\sum_{\tau \neq \sigma, \gamma} m_{\tau} \gamma\left(b_{\tau}\right)=1-1+3=n_{\gamma} .
\end{array}\right.
$$

Se $\tau \in \mathcal{A} s s(A)-\{\sigma, \gamma\}$ e $e_{\tau}=1$, então

$$
\begin{aligned}
\tau(b) & =\tau\left(b_{0}\right)+\tau\left(q_{\sigma}\right)+\tau\left(q_{\gamma}\right)-m_{\tau} \tau\left(b_{\tau}\right)= \\
& =n_{\tau}-1-1-4 m_{\tau}^{\prime}= \\
& =2 m_{\tau}^{\prime}+1-2-4 m_{\tau}^{\prime}= \\
& =-2 m_{\tau}^{\prime}-1=-n_{\tau} .
\end{aligned}
$$

Se $\tau \in \mathcal{A} s s(A)-\{\sigma, \gamma\}$ e $e_{\tau}=-1$, então

$$
\begin{aligned}
\tau(b) & =\tau\left(b_{0}\right)+\tau\left(q_{\sigma}\right)+\tau\left(q_{\gamma}\right)-m_{\tau} \tau\left(b_{\tau}\right)= \\
& =-n_{\tau}-2-4\left(-\left(m_{\tau}^{\prime}+1\right)\right)= \\
& =n_{\tau} .
\end{aligned}
$$

Assim, $|\tau(b)|=n_{\tau}$, para todo $\tau \in \mathcal{A s s}(A)$, como queríamos.

Corolário 4.21 Se valem as condições equivalentes do teorema anterior, entäo vale a fatoração única em irredutíveis para formas de dimensão ímpar em $\mathcal{W}(A)$. 
Dem.: Mostra-se de maneira análoga a demonstração canônica de que todo domínio de ideais principais é um domínio fatorial, veja por exemplo [11]. 


\section{Referências Bibliográficas}

[01] ATrYAH, M.F. e MACDONALD, I.G.; Introduction to Commutative Algebra, University of Oxford, 1969.

[02] BAEZA, R.; Quadratic Forms over Semilocal Ring, Lecture Notes in Mathematics $655,1978$.

[03] BRUSAMARELLO, R.; Ideais Primos do Anel de Witt sobre um Anel Local, Tese de Mestrado, ICMSC-USP, 1991.

[04] DIAS, I.; Formas Quadráticas sobre LG-anéis, Tese de Doutorado, IMECCUNICAMP, 1988.

[05] FITZGERALD, R.W.; Primary Ideals in Witt Rings, Communication in Algebra 96, 368-385 (1985).

[06] FITZGERALD, R.W.; Ideal Class Groups of Witt Rings, Journal of Algebra 124, 506-520 (1989).

[07] KAPLANSKY, I.; Commutative Rings, Allyn an Bacon, Boston, 1970.

[08] KNEBUSH, M.;ROSENBERG, A.; WARE,R.; Struture of Witt Rings, Quotients of Abelian Group Rings, and Orderings of Fields, Bull. Amer. Math. Soc. 77, 208-210, (1971).

[09] KNEBUSCH, M.; ROSENBERG, A.; WARE, R.; Struture of Witt Ring and Quotients of Abelian Group Rings, Amer. J. of Math. 94, 119-155, (1972). 
[10] LAM, T. Y.; The Algebric Theory of Quadratic California, 1973.

[11] LANG, S.; Algebra, Addison-Wesley P.C., Inc., 1993.

[12] MCDONALD,B.R.; Linear Algebra over Commutative Rings; Pure and Applied Math, 87, Marcel Dekker,INC., 1984.

[13] PIERCE, R.; Associative Algebras, G. T. M. 88, Springer Verlag, 1982. 